University of Louisville ThinkIR: The University of Louisville's Institutional Repository

Electronic Theses and Dissertations

$5-2016$

\title{
Synthesis, characterization, and electronic properties of novel 2D materials : transition metal dichalcogenides and phosphorene.
}

George Anderson

University of Louisville

Follow this and additional works at: https://ir.library.louisville.edu/etd

Part of the Condensed Matter Physics Commons, and the Semiconductor and Optical Materials Commons

\section{Recommended Citation}

Anderson, George, "Synthesis, characterization, and electronic properties of novel 2D materials : transition metal dichalcogenides and phosphorene." (2016). Electronic Theses and Dissertations. Paper 2428.

https://doi.org/10.18297/etd/2428

This Doctoral Dissertation is brought to you for free and open access by ThinkIR: The University of Louisville's Institutional Repository. It has been accepted for inclusion in Electronic Theses and Dissertations by an authorized administrator of ThinkIR: The University of Louisville's Institutional Repository. This title appears here courtesy of the author, who has retained all other copyrights. For more information, please contact thinkir@louisville.edu. 
SYNTHESIS, CHARACTERIZATION, AND ELECTRONIC PROPERTIES OF NOVEL 2D MATERIALS: TRANSITION METAL DICHALCOGENIDES AND PHOSPHORENE

\author{
By \\ George Anderson, \\ B.S. Eastern Kentucky University \\ M.S., University of Louisville
}

\begin{abstract}
A Dissertation
Submitted to the Faculty of the College of Arts and Sciences of the University of Louisville

In Partial Fulfillment of the Requirements

For the Degree of
\end{abstract}

Doctor of Philosophy in Physics

Department of Physics

University of Louisville

Louisville, Kentucky

May 2016 

SYNTHESIS, CHARACTERIZATION, AND ELECTRONIC PROPERTIES OF NOVEL 2D MATERIALS: TRANSITION METAL DICHALCOGENIDES AND PHOSPHORENE

\section{By}

George Anderson, BS., Eastern Kentucky University
M.S., University of Louisville

A Dissertation Approved on

April 15, 2016

By the Following Dissertation Committee:

Dr. Gamini Sumanasekera (Thesis Director)

Dr. C.S. Jayanthi

Dr. Ming Yu

Dr. Xiao-An Fu 


\section{ACKNOWLEDGEMENTS}

To attend graduate school and become a doctor has been a blessing from Jesus Christ and means more to me than I can ever explain. I thank the University of Louisville's physics department for giving me this amazing opportunity. In particular I thank Dr. Gamini Sumanasekera for being an amazing advisor and friend. His experience, guidance, and willingness to work with me is one of the main reasons I am graduating. Words cannot express how thankful I am to have a department chair such as Dr. C.S. Jayanthi. Even when I wanted to quit she did not give up on me and pushed me to work harder. She along with Dr. Chris Davis have worked extremely hard to ensure I was always funded and were always available when I needed someone to talk too. Dr. Ming Yu has greatly impacted my life by first introducing me to research and showing me how I can succeed in high level courses. I thank Dr. Xiao-An Fu for being a part of my committee in addition to providing advice and guidance that has improved my research. With all my heart, I thank Dr. Shi-Yu Wu and his wife for visiting me when I was in China doing research. Thanks to Dr. Xin Zihua and Dr. Ming Yu, I was able to do research in China. I am thankful for the guidance that Dr. Jacek Jasinski, Dr. Tatiana Krentsel, and Dr. Tereza Paronyan have given me. It has truly shaped the way I do research and their door was always open to anyone who needed their advice. Due to Dr. Humberto Rodriguez Gutierrez, I have developed a strong understanding of what it means to be a scientist. The support staff for the physics department has to be some of the best people I know. Mary Gayle Wrocklage, Rea Diehlmann, and Joshua Rimmer are truly amazing 
people. I thank my lab partners Rong Zhao, Meysam Akhtar, Adel Alruqi, Ruchira Dharmasena, Congyan Zhang, and Cherno Kah. I have learned not only science from them, but also I have a much greater appreciation for their cultures and those differences that make them special people. I would not have been able to become a doctor if not for the support and guidance of all these amazing people. I am also thankful for the McSwneey Fellowship of the College of Arts and Sciences. The fellowship allowed me to be able to attain my doctoral degree. I thank my amazing wife. She has stood by my side and supported me through extremely hard times as we pursued this $\mathrm{PhD}$. She is the love of my life. I thank my kids. They were the motivation I needed to pursue a higher education. I hope they know that they can accomplish their goals and dreams regardless of what this world tells them. I thank my mother and step father for all their support and hard work. They pushed me to do better and showed me how to work hard. 


\section{ABSTRACT \\ SYNTHESIS, CHARACTERIZATION, AND ELECTRONIC PROPERTIES OF NOVEL 2D MATERIALS: TRANSITION METAL DICHALCOGENIDES AND PHOSPHORENE}

George Anderson

$15^{\text {th }}$ April 2016

Scaling electronic devices has become paramount. The current work builds upon scaling efforts by developing novel synthesis methods and next generation sensing devices based on 2D materials. A new combination method utilizing thermal evaporation and chemical vapor deposition was developed and analyzed to show the possibilities of Transition Metal Dichalcogenide monolayers and heterostructures. The materials produced from the above process showed high degrees of compositional control in both spatial dimensions and chemical structure. Characterization shows controlled fabrication of heterostructures, which may pave the way for future band gap engineering possibilities. In addition, Phosphorene based field effect transistors have been developed to show the versatility and potential of $2 \mathrm{D}$ based optoelectronic devices. As phosphorene has been found to be a potential candidate for real world field effect transistors, the interplay between device performance and environmentally conditions have been studied using in-situ transport measurements. Phosphorene was exposed to varying light and gaseous environments to understand how it preforms under different external stimuli. It was found that both light and gases can have extreme impacts on the properties of phosphorene. 
TABLE OF CONTENTS

PAGE

ACKNOWLEDGEMENTS .........................................................................................

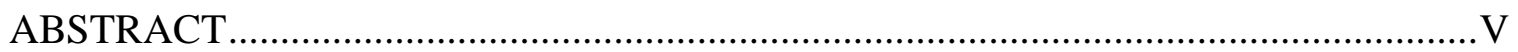

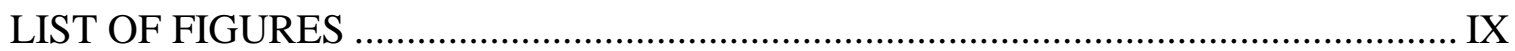

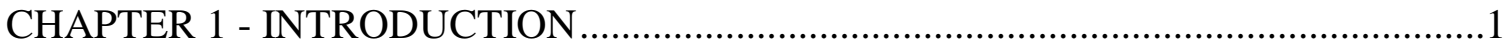

1.1 HISTORY OF TRANSITION METAL DICHALCOGENIDES ..............................

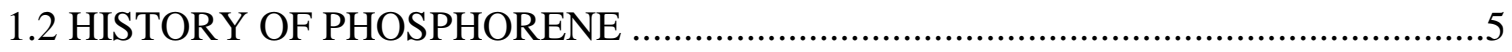

1.3 PHOSPHORUS ATOM AND ITS HYBRIDIZATIONS …...................................

1.4 BAND STRUCTURE OF PHOSPHORENE ........................................................

1.5 SYNTHESIS OF TRANSISTION METAL DICHALCOGENIDES AND

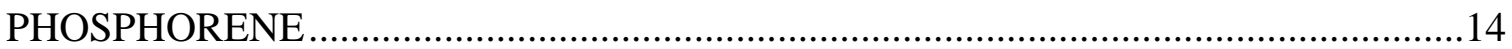

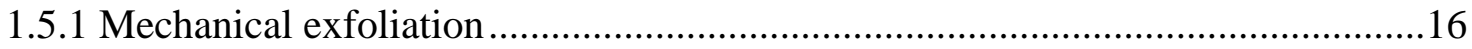




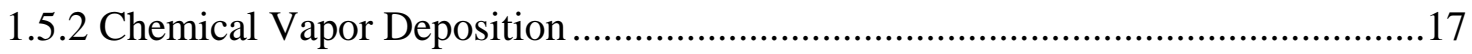

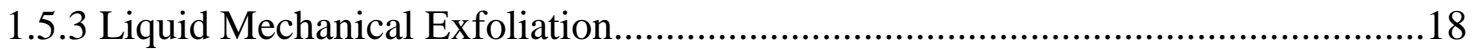

1.6 Modifications of Transition Metal Dichalcogenides and Phosphorene ......................20

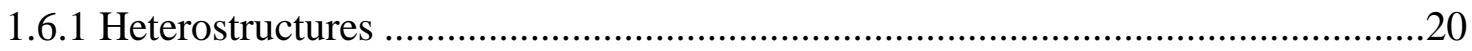

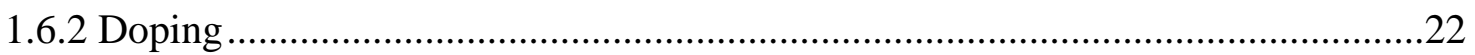

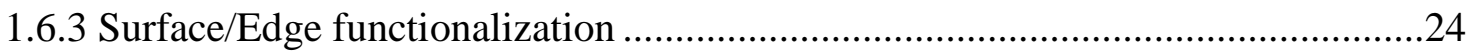

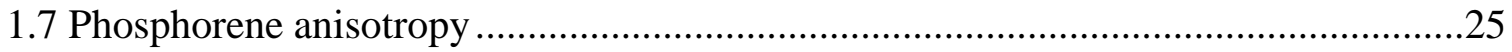

CHAPTER 2 - SYNTHESIS AND CHARACTERIZATION OF TRANSITION METAL

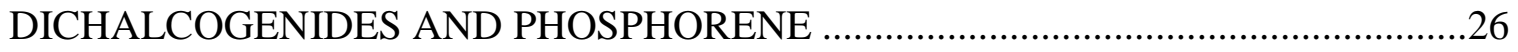

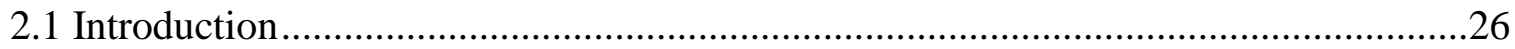

2.2 Thermal evaporation and CVD Kinetics and transport............................................27

2.3 Synthesis of Transition Metal Dichalcogenides Heterostructures ................................38

2.4 Transfer of Transition Metal Dichalcogenide Heterostructures ..................................47

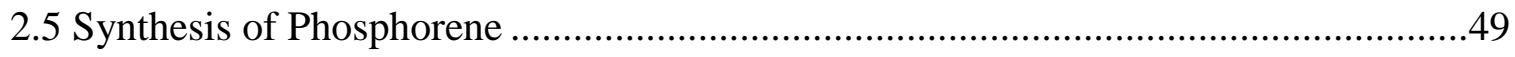

2.6 Transition Metal Dichalcogenide Characterization .....................................................54

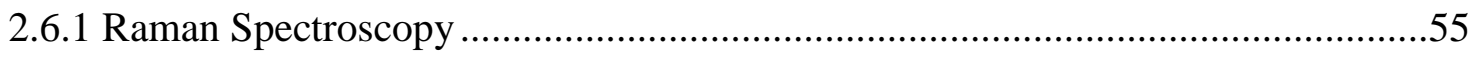

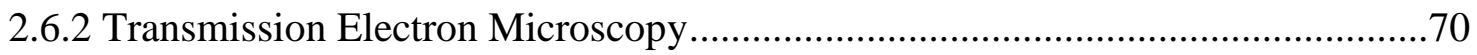

2.7 Phosphorene Characterization ..............................................................................72

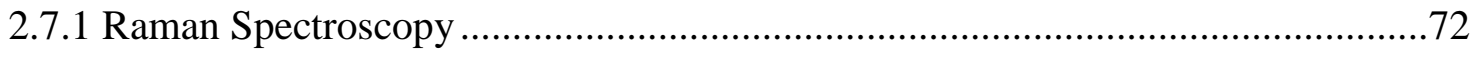


2.7.2 Photoluminescence

2.7.3 Scanning Electron Microscopy ………….....................................................77

2.7.4 Transmission Electron Microscopy ……………............................................78

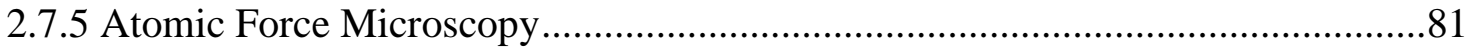

CHAPTER 3 - PHOSPHORENE BASED OPTOELECTRONIC DEVICES ...................83

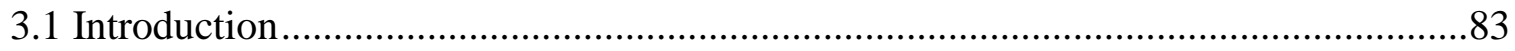

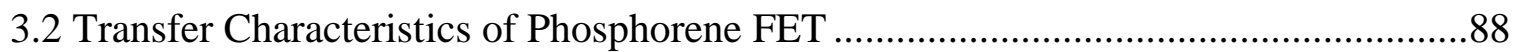

3.3 In-situ environment dependent transport in phosphorene..........................................89

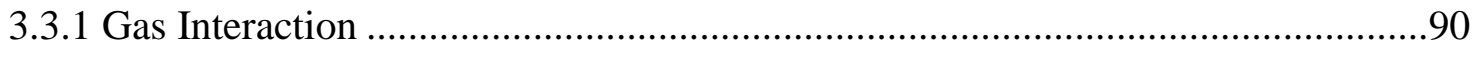

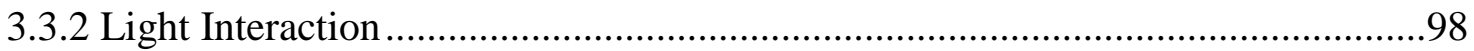

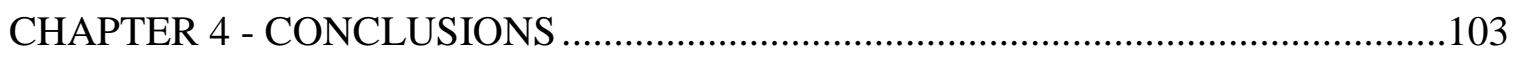

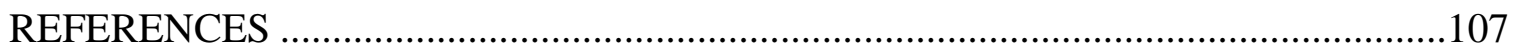

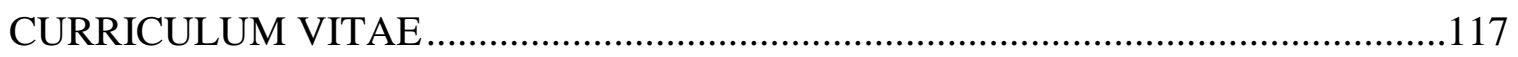




\section{LIST OF FIGURES}

Page

FIGURE 1.1 LIST OF CHALCOGEN ATOMS AND SOME TRANSITION METAL ATOMS............... 1

FIGURE 1.2 STRUCTURE OF MONOLAYER TRANSITION METAL DICHALCOGENIDE .............. 2

FIGURE 1.3 GROUND STATE ELECTRONIC CONFIGURATION FOR PHOSPHORUS .................... 7

FigURE 1.4 A.) STRUCTURE OF PHOSPHORENE TOP VIEW. B.) STRUCTURE OF

PHOSPHORENE SIDE VIEW WITH BUCKLING PARAMETER ............................................ 9

FIGURE 1.5 STABILITY AND CONDUCTIVITY OF SEVERAL MONOLAYER TMD'S............... 15

FIGURE 1.6 SCHEMATIC OF MECHANICAL EXFOLIATION PROCESS.................................... 16

FIGURE 1.7 SCHEMATIC OF MULTI-ZONE QUARTZ TUBE FURNACE USED FOR CVD. THIS

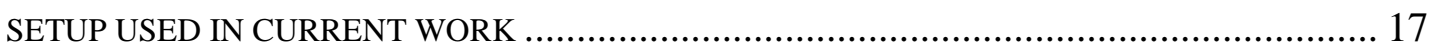

FIGURE 1.8 MULTI-ZONE QUARTZ TUBE FURNACE...................................................... 17

FIGURE 2.1 A.) SCHEMATIC OF THERMAL EVAPORATION PROCESS. B.) AND C.)

REFURBISHED THERMAL EVAPORATOR. D.) AND E.) INSIDE VACUUM CHAMBER......... 28

FIGURE 2.2 A.) SCHEMATIC OF THERMAL EVAPORATION DONE AT STANDARD ATMOSPHERIC PRESSURES. B.) SCHEMATIC OF THERMAL EVAPORATION DONE AT LOW

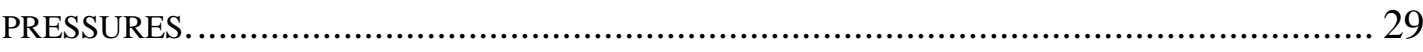

FIGURE 2.3 SCHEMATIC OF GROWTH PROCESS FOR HETEROSTRUCTURES MADE BY SERIES A AND SERIES B METHODS. INSET IS OF PREDICTED STRUCTURES FORMED AFTER

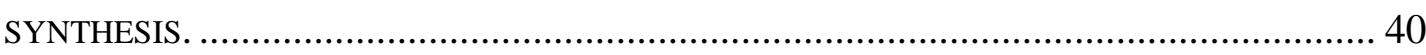

FIGURE 2.4 TEMPERATURE PROFILE USED DURING CHALCOGENIZATION OF SULFUR BASED TMD's. 42

FIGURE 2.5 TEMPERATURE PROFILE USED DURING CHALCOGENIZATION OF SELENIUM

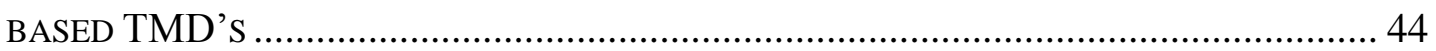

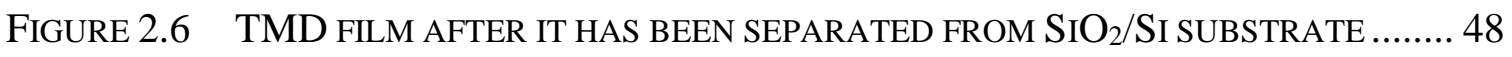

FIGURE 2.7 PRECURSOR MATERIAL FOR BULK BLACK PHOSPHORUS SYNTHESIS INSIDE GLASS TUBE DURING EVACUATION PROCESS .......................................................... 49

FIGURE 2.8 A.) AND B.) OXYGEN AND ACETYLENE TANKS USED FOR GLASS SEALING. C.)

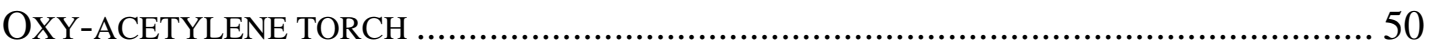

FIGURE 2.9 CUSTOM VACUUM SYSTEM FOR EVACUATING GLASS TUBES ......................... 50

FIGURE 2.10 SINGLE ZONE QUARTZ TUBE FURNACE WITH VIAL OF PRECURSORS ............... 51

FIGURE 2.11 TEMPERATURE PROFILE OF FURNACE USED IN BULK BLACK PHOSPHORUS

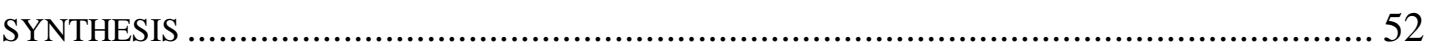

FIGURE 2.12 GLASS TUBE AFTER GROWTH OF BULK BLACK PHOSPHORUS ........................ 53

Figure 2.13 Optical IMAges Of MONOlayer A.) $\mathrm{MoS}_{2}$, B.) $\mathrm{MoSE}_{2}$, C.) MoTe 2 AND D.)

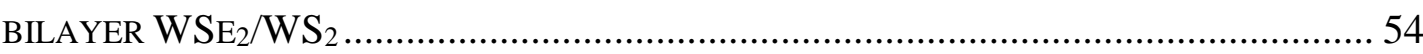

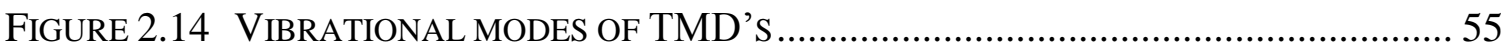

FIGURE 2.15 SCHEMATIC OF RAMAN PROCESS .............................................................. 57

FIGURE 2.16 RAMAN SPECTRUM OF MONOLAYER WSE2 EXPOSED TO LASER SEVERAL TIMES 60 
FIGURE 2.17 A.) RAMAN OF BILAYER $\mathrm{WS}_{2} / \mathrm{MOS}_{2}$ B.) RAMAN OF MONOLAYER $\mathrm{WS}_{2}$ ON 4 LAYER $\mathrm{MOS}_{2}$ C.) OPTICAL IMAGE SHOWING LOCATIONS USED FOR RAMAN MEASUREMENTS. D.) RAMAN MAPPING OF $\mathrm{WS}_{2} \mathrm{E}_{2 \mathrm{G}}^{1}$ E.) RAMAN MAPPING OF $\mathrm{MOS}_{2} \mathrm{E}_{2 \mathrm{G}}^{1}$ 61

Figure 2.18 DECONVOLUTED RAMAN OF A.) BILAYER $\mathrm{WS}_{2} / \mathrm{MOS}_{2}$ SERIES A. B.) BILAYER $\mathrm{WS}_{2} / \mathrm{MOS}_{2}$ SERIES B.

FIGURE 2.19 A.) RAMAN OF BILAYER WSE2/MOSE2 B.) RAMAN OF MONOLAYER WSE2 ON 4 LAYER MoSE 2 C.) RAMAN MAPPING OF WSE2 DEGENERATE $E^{1}{ }_{2 G^{-}} A_{1 G}$ D.) RAMAN MAPPING OF MOSE $2 \mathrm{E}_{2 \mathrm{G}}^{1}$

FIGURE 2.20 A.) RAMAN SPECTRUM OF BILAYER WSE2/WS 2 B.) OPTICAL IMAGE OF

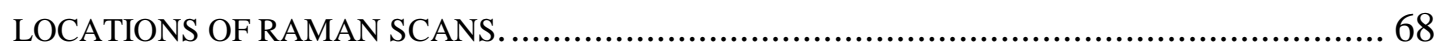

FIGURE 2.21 OPTICAL IMAGE OF BILAYER WSE $2 / \mathrm{WS}_{2}$ WITH CROSS LINKING PATTERN..... 69 Figure 2.22 A.) LOW MAGNIFICATION TEM IMAGE OF A SERIES-A WS $2 / \mathrm{MOS}_{2}$

HETEROSTRUCTURE. B.) AND C.) ELECTRON DIFFRACTION PATTERNS IN THE SQUARE AND OUTSIDE THE SQUARE RESPECTIVELY. (D) HRTEM AND FT OF NANO-CRYSTAL DOMAINS FOR 15 MIN CHALCOGENIZATION. (E) CROSS-SECTION VIEW OF THE TMD BILAYER ON THE SQUARE. (F) HETEROSTRUCTURE TRANSFERRED TO TEM/LACEY CARBON GRID 71

FIGURE 2.23 VIBRATIONAL MODES OF PHOSPHORENE ................................................ 72

FIGURE 2.24 RAMAN SPECTRUMS OF BULK BLACK PHOSPHORUS, BULK RED PHOSPHORUS, AND PHOSPHORENE FROM DIFFERENT LOCATIONS OF CENTRIFUGE VIAL..................... 73

FIGURE 2.25 SCHEMATIC OF PL PROCESS ................................................................ 74

FIGURE 2.26 PL FROM BULK BLACK PHOSPHORUS AND PHOSPHORENE FROM DIFFERENT

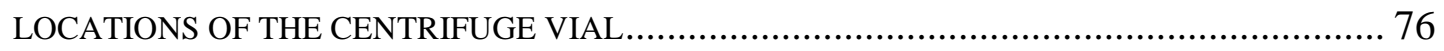

FIGURE 2.27 A.) AND B.) SEM IMAGES OF PHOSPHORENE............................................. 77

FIGURE 2.28 A.) ELECTRON DIFFRACTION OF PHOSPHORENE. B.) TEM OF PHOSPHORENE C.)

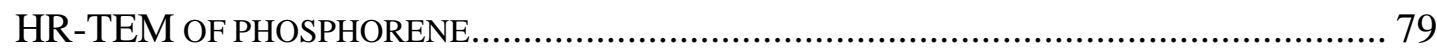

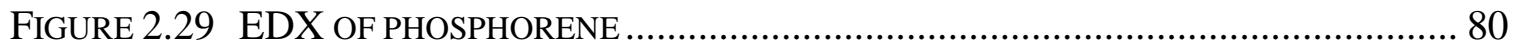

FIGURE 2.30 AFM OF PHOSPHORENE....................................................................... 81

FIGURE 2.31 3D RENDERED AFM OF PHOSPHORENE ................................................. 82

FIGURE 3.1: SCHEMATIC OF FIELD EFFECT TRANSISTOR .......................................... 83

FIGURE 3.2: P-TYPE FIELD EFFECT TRANSISTOR A.) WITH REVERSE-BIASED AND B.)

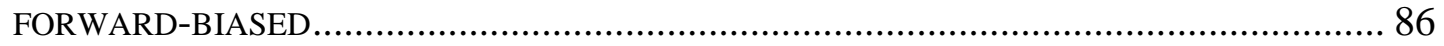

Figure 3.3: A.) PhOSPhORENE FET ON PROBE. B.) PHOSPHORENE FET INSIDE VACUUM SYSTEM. C.) IN-SITU SETUP. D.) ELECTRODE PATTERN ON CHIP............................... 89

FIgURE 3.4: PHOSPHORENE FET I-V CURVES FOR VG $=20 \mathrm{~V}$ (BLACK), $0 \mathrm{~V}$ (BLUE), AND -10 $\mathrm{V}$ (RED) 90

FIGURE 3.5: RELATIVE CHANGE IN THE RESISTANCE OF PHOSPHORENE AS IT IS HEATED AND COOLED VERSUS TIME.

FIGURE 3.6 RELATIVE CHANGES IN THE RESISTANCE OF PHOSPHORENE AS IT IS HEATED AND COOLED VERSUS TEMPERATURE.

FIGURE 3.7: RELATIVE CHANGE IN RESISTANCE IN PHOSPHORENE AS IT IS EXPOSED TO VARIOUS GASES AT $60^{\circ} \mathrm{C}$. 
FIGURE 3.8: TRANSFER CHARACTERISTICS OF PHOSPHORENE AS IT IS EXPOSED TO VARIOUS

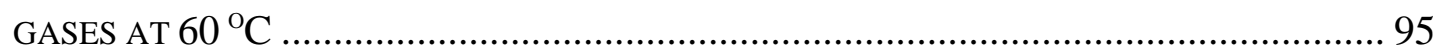

FIGURE 3.9: SCHEMATIC OF BAND DIAGRAM SHOWING HOW THE SCHOTTKY BARRIER IS FORMED BETWEEN PHOSPHORENE AND AL CONTACTS. .......................................... 96

FIGURE 3.10: TRANSFER CHARACTERISTICS OF PHOSPHORENE AS IT IS EXPOSED TO VARIOUS LED'S 99

FIGURE 3.11: HOLE MOBILITY IN PHOSPHORENE FOR EXPOSURE TO DIFFERENT LED'S . 99 FIGURE 3.12 RELATIVE RESISTANCE OF PHOSPHORENE EXPOSED TO RED AND YELLOW LEDs. 100

FIGURE 3.13: SCHEMATIC OF BAND DIAGRAM OF A.) PRISTINE PHOSPHORENE B.) AND PHOSPHORENE EXPOSED TO LIGHT WITH ENERGY GREATER THAN PHOSPHORENE'S BAND GAP. 101

FIGURE 3.14: RELATIVE CHANGES IN RESISTANCE FOR PHOSPHORENE EXPOSED TO A.) YELLOW LED AND B.) RED LED FOR DIFFERENT LED VOLTAGES............................. 102 


\section{CHAPTER 1 - INTRODUCTION}

\subsection{HISTORY OF TRANSITION METAL DICHALCOGENIDES}

Transition metal dichalcogenides encompass a very diverse group of materials. They are defined as a transition metal atom attached to two atoms from Group 16 of the periodic table [1-4]. Figure 1.1, shows some of the elements in transition metal dichalcogenides (TMD).

\section{$M=$ Transition Metal}

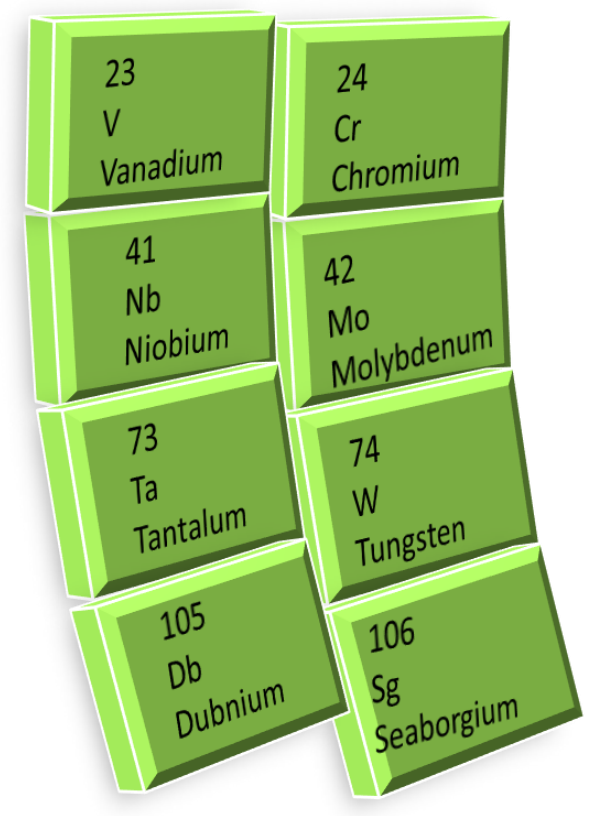

Figure 1.1

$$
X=\text { Chalcogen }
$$

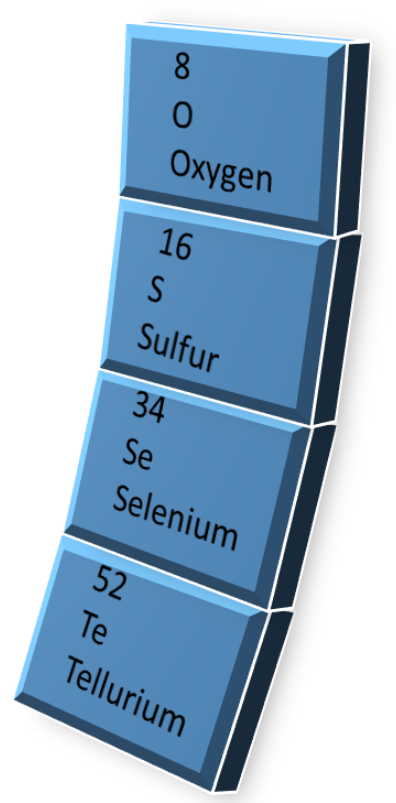

Historically, they have been used as additives to lubricants as they decrease friction [5, 6]. However, with the advent of graphene in 2004 [7], they have gained new 
interest. TMD's also have been shown to have catalytic abilities $[8,9]$. Monolayer $\mathrm{MoS}_{2}$ has been shown to have highly active catalytic sites only at its edges and its basal plane is catalytically inert [10-12]. In addition remarkable optical properties have been observed, such as room temperature photoluminescence in a single layer of $\mathrm{WS}_{2}[13]$ and $\mathrm{WSe}_{2}$ $[14,15]$.

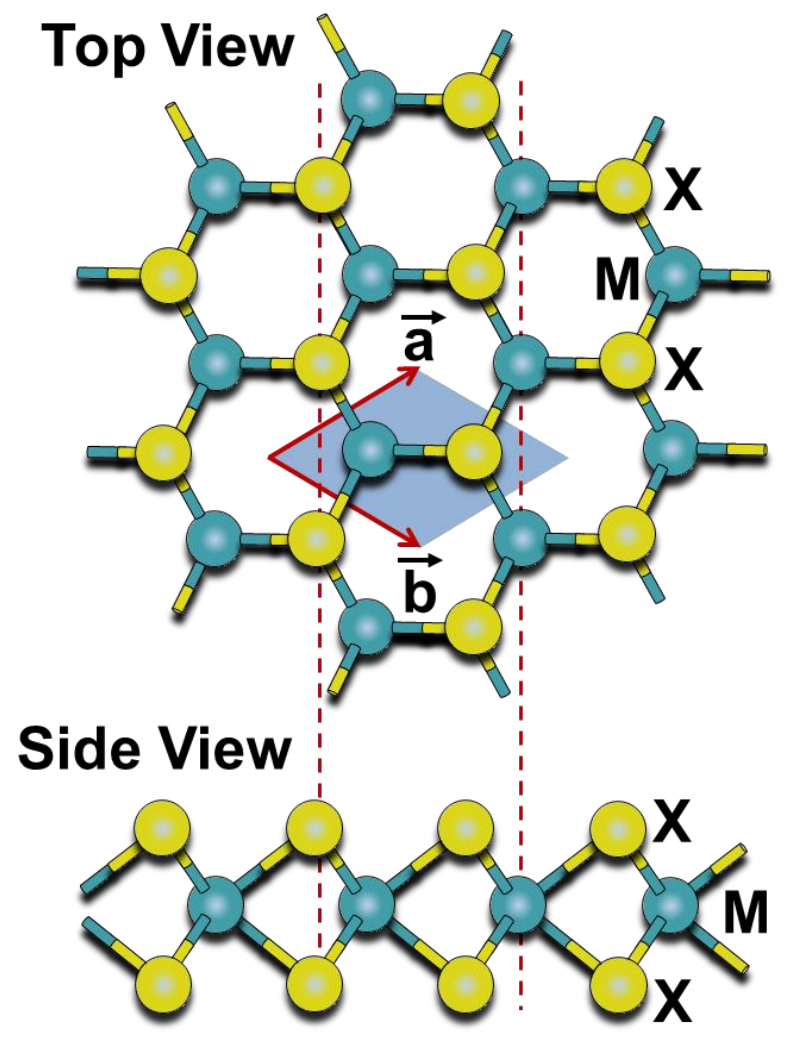

Figure 1.2 Structure of monolayer transition metal dichalcogenide 
TMD's are some of the rare compounds in which the bulk material is composed of individual layers of material stacked vertically with respect to each other. Each layer has hexagonal or rhombohedral symmetry [16-18] and can be viewed as a layer of transition metal atoms sandwiched between two layers of chalcogen atoms (figure 1.2).

In TMD's, it was found that these individual layers could be separated using a mechanical exfoliation process that allowed for stable single layer TMD's to be synthesized [19-21]. Once the above was discovered, publications in TMD systems increased drastically. Monolayer TMD's can be metallic, insulating, or semiconducting $[4,22,23]$. They have various properties and can be tuned based on the number of layers present in the sample. Photoluminescence, conductivity, band structures, and vibrational modes can all be altered by varying layering number $[1,13,21,24-28]$. The monolayer form and tunable properties has made TMD's a very promising material. For instance, a transition from indirect band gap (in bulk) to direct band gap (in single-layer form) is responsible for the existence of photoluminescence (PL) at room temperature in monolayer TMD's $[13,29,30]$. The phonon modes are also altered in single-layer sheets since the lack of inter-layer van der Waals interaction modifies the restoring forces of the lattice vibrations $[24,31,32]$.

Some of the most popular TMD's are the $\mathrm{MX}_{2}$ grouping with $\mathrm{M}=\mathrm{Mo}, \mathrm{W}$, and $\mathrm{X}$ $=\mathrm{S}, \mathrm{Se}, \mathrm{Te}$. Molybdenum has 42 electrons with electronic configuration $1 s^{2} 2 s^{2} 2 p^{6} 3 s^{2} 3 p^{6} 3 d^{10} 4 s^{2} 4 p^{6} 4 d^{5} 5 s^{1}$. Sulfur has 16 electrons with $1 s^{2} 2 s^{2} 2 p^{6} 3 s^{2} 3 p^{4}$ configuration. Inplane bonding is covalent and is due to the $4 \mathrm{~d}$ orbital of molybdenum and the $3 p$ orbital of sulfur [33]. However, it is the out of plane bonding that is responsible for most of the interest in TMD's. Each layer interacts with adjacent layers 
through van der Waals forces. This attraction is extremely weak (15-20 meV/ $\left.\AA^{2}\right)$ [34] compared to the inplane forces, which is the reason TMD's can be fabricated in monolayer form.

In monolayer form, their band gaps span from $1.0 \mathrm{eV}$ to $2.5 \mathrm{eV}$ [35-37]. $\mathrm{MoS}_{2}$ has a direct monolayer band gap between 1.2-1.8 eV [36] and an indirect bulk band gap of $1.06 \mathrm{eV}$ [38]. For $\mathrm{WS}_{2}$ the reason that the band gap increases as the number of layers decrease is due to a decrease in energy at the $\Gamma$ point and an increase in energy at the $\mathrm{K}$ point in the energy dispersion relation [13]. As more emphasis is placed on scaling electronic devices, TMD's have come to the top of the pack. Due to the TMD family having small lattice mismatches and being a van der Waals material, many different types of heterostructures can be synthesized [39-53]. The above structures allow for band gap engineering, absorption enhancement, improved charge separation, and many other possibilities. These properties allow for some of the most novel optoelectronic devices to be fabricated. Field effect transistors, photocatalysis, photovoltaics, and logic circuits have all be studied. The physics in these monolayer compounds has proven to be very rich and numerous device demonstrations at the laboratory scale [26, 43, 54-60] support their potential for practical applications. Vertically stacked heterostructures (VSH) of graphene/h-BN have been synthesized [61, 62]; and graphene/MoS $2 /$ graphene and graphene/WS2/graphene have been used to demonstrate photoactive devices [55]. Additionally, $\mathrm{MoS}_{2} / \mathrm{Graphene} / \mathrm{BSCO}$ heterostructures have been assembled for logic transistors [43]. Theoretical calculations suggest that it is possible to obtain a direct band gap layered material with the right combination of alternated TMD sheets [63]. Particularly, when the chalcogen atom is different in the adjacent layers, the photo- 
generated electrons and holes could be physically separated and localized in neighboring layers [63]. In fact, $\mathrm{WSe}_{2} / \mathrm{MoS}_{2}$ heterojunctions [64-66] with photovoltaic behavior [65] have been recently prepared by direct transfer of TMD flakes. Lateral (in-plane) heterostructures of continuous $2 \mathrm{D}$ crystals have proven to be more difficult to produce. From a theoretical standpoint the band alignments for a variety of TMD-based 2D heterojunctions suggest potential applications in water splitting [3]. The band offsets have been found to be extremely sensitive to the number of layers, making possible the fabrication of quantum structures by thickness engineering.

\subsection{HISTORY OF PHOSPHORENE}

In order to detail the path that phosphorene has taken, it is important to start with its bulk counterpart. Black phosphorus was first discovered in 1914 [67]. Phosphorus has four main allotropes and many more modification subsets of each one [68]. The most popular of these allotropes are red, white, and black. Red and white phosphorus are highly flammable unlike black phosphorus which is the most stable of the allotropes [67, 69-74]. Bulk black phosphorus forms with several crystalline structures including orthorhombic, rhombohedral, and cubic $[73,75]$. Orthorhombic black phosphorus with space group Cmca [73] is of importance because of its layered structure. Similar to graphite, orthorhombic black phosphorus is formed by repeated vertical stacking of individual atomic layers. The bonding of each atomic layer is very anisotropic. Out of plane interactions between each layer is weak (exfoliation energy is about -151 meV/atom) [76] and is considered a consequence of van der Waals interactions. As such, black phosphorus is also known as a van der Waals material which includes the 
previously introduced TMD's and graphite. In-plane bonding is much stronger and is governed by atomic orbital hybridization. One of the most striking properties of black phosphorus is its high hole mobility. In addition the energy band gap of bulk black phosphorus is $0.3 \mathrm{eV}$, which places it in the infer-red region of the electromagnetic spectrum [77].

Bulk black phosphorus has been historically synthesized by high pressure routes $[67,71,72,74]$. P. W. Bridgman found that when white phosphorus was subject to a 1.2 GPa pressure, that the phosphorus underwent a structural phase change. The resulting change was black phosphorus. Both white and red phosphorus can be induced to transform into black phosphorus at high pressures $[71,74]$. However, high pressure routes of synthesis have relatively small yields and the high pressures make experimental design a safety issue. As more interest in black phosphorus occurred, more methods of synthesis have been developed. Bulk black phosphorus has been made by high speed ball milling [78], closed transport reactions $[79,80]$ and most recently through high energy sonication [81].

Increased interest of black phosphorus is in part due to the discovery of graphene in 2004 [7], just like TMD's. The successful separation of graphite layers to form one layer of graphene using mechanical exfoliation [7, 82] sparked an arms race for 2D and quasi-2D materials. The amount of attention that black phosphorus received paid off in 2014 when two teams successfully synthesized monolayer black phosphorus $[83,84]$. Named phosphorene, monolayer black phosphorus has many amazing properties. It has variable hole mobility ranging from $1000 \mathrm{~cm}^{2} / \mathrm{Vs}$ down to $14 \mathrm{~cm}^{2} / \mathrm{Vs}$ [83-91]. Just like graphene it has free standing capabilities, and can be synthesized using many of the same 
techniques. Phosphorene also has a $2 \mathrm{eV}$ band gap [89, 92], which places it above gapless graphene and $1.9 \mathrm{eV}$ of $\mathrm{MoS}_{2}[19]$.

\subsection{PHOSPHORUS ATOM AND ITS HYBRIDIZATIONS}

Phosphorus is in Group 15 of the periodic table. It has 16 neutrons, 15 electrons and 15 protons. Phosphorus compounds are abundant and found in many different systems. Groups of phosphates $\left(\mathrm{PO}_{4}{ }^{3-}\right)$ are found in adenosine triphosphate better known as ATP [93]. ATP is used as an energy source and as a neurotransmitter in our bodies [93].

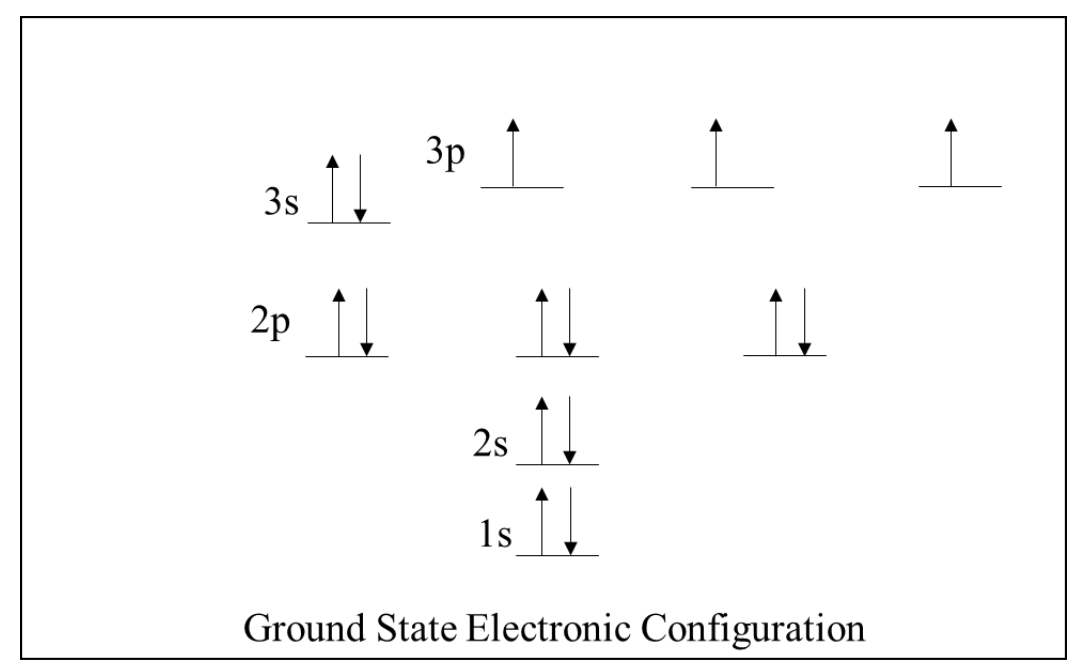

Figure 1.3 Ground state electronic configuration for phosphorus

Electronic configuration of phosphorus atoms in the ground state is $1 s^{2} 2 s^{2} 2 p^{6} 3 s^{2} 3 p^{3}$. The orbitals involved in chemical reactions are the $3 s$ and $3 p$ orbitals shown in figure 1.3. These orbitals are responsible for the nonexistence of elemental phosphorus naturally occurring. Unlike graphite, in-plane bounding in black phosphorus is due to $\mathrm{sp}^{3}$ hybridization [94]. Each phosphorus atom is bonded to three adjacent 
phosphorus atoms. The above requires that each phosphorus atom retains a lone pair of electrons. Due to this bonding, phosphorene does not form atomically flat sheets like graphene. Instead it forms a puckered honeycomb structure. There are several defined symmetry directions in phosphorene. They are defined as the zigzag and armchair directions. Having a puckered structure gives phosphorene very unique properties. There is built in anisotropy for strain, mobility, diffusion, and magnetic properties along with others [90, 95-113] along the zigzag and armchair directions.

Due to each phosphorus atom having a lone pair of electrons, black phosphorus is a highly reactive material [114]. Those lone pairs contribute to bonding with other elements, in particular water and oxygen. In the presence of ambient environmental conditions phosphorene degrades to $\mathrm{PO}_{x}$ species [115] and phosphoric acid $\mathrm{H}_{3} \mathrm{O}_{4} \mathrm{P}$ [116] within a few hours. Much work has been made to stop degradation in phosphorene. Capping layers and advanced synthesis routes seem to be very promising $[87,117,118]$.

\subsection{BAND STRUCTURE OF PHOSPHORENE}

In order to calculate the band structure of phosphorene several methods are used. Density functional theory (DFT) has usually been employed because it is an exact formulation [119-121]. However, the exchange-correlation energy functional in DFT is usually approximated because it is computationally expensive. As such, exchangecorrelation functional approximations are incorporated. These include GGA generalized gradient approximations [122], LDA local-density approximations [123] and hybrids [124] among others. 
Unlike $\mathrm{sp}^{2}$ hybridization materials like graphene, phosphorene's $\mathrm{sp}^{3}$ hybridization increases the interlayer spacing between adjacent monolayers more. In addition, each layer has an influence on adjacent layers inducing self-alignment, which maintains the built in anisotropic structure of phosphorene even up to the bulk level [83]. Phosphorene has a layer dependent band gap just like the TMD's.

\section{Top View}

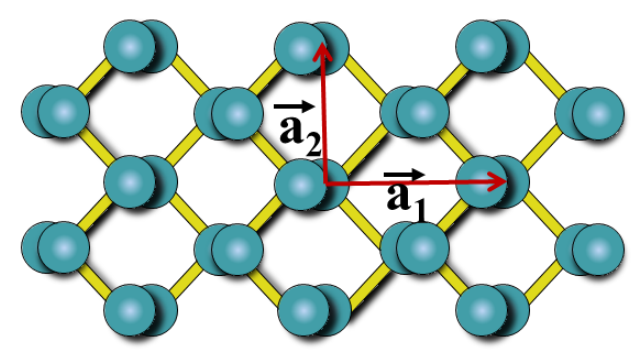

Side View

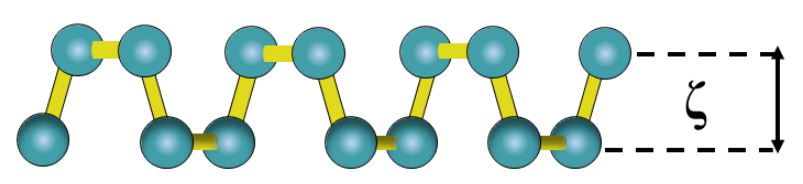

Figure 1.4 parameter

a.) Structure of phosphorene top view. b.) Structure of phosphorene side view with buckling

For phosphorene, the buckled/puckered hexagonal crystal structure is shown in figure 1.4 with $\overrightarrow{a_{1}}$ and $\overrightarrow{a_{2}}$ being the lattice vectors. Armchair direction lines up with the xaxis (horizontal) and $\overrightarrow{a_{1}}$. Zigzag direction lines up with the y-axis and $\overrightarrow{a_{2}}$. Phosphorene has four atoms in its unit cell [104]. Also the buckling parameter is shown in figure 1.4. It is found to be $0.25 \mathrm{~nm}$ from hybrid exchange-correlation functional HSE06 calculations $[125,126]$. Buckling parameters are defined as the vertical distance that separates the covalently bonded atoms in the structure $[84,125]$ along the z-axis. The origin of the lattice vectors is placed at the center of an atom on the lower plane of the buckle. By 
defining the z-axis to be out of plane, the magnitude of its lattice vector is equal to the buckling parameter $\zeta$. The lattice vectors are defined in terms of the $\mathrm{x}, \mathrm{y}, \mathrm{z}$ coordinates as follows,

$$
\overrightarrow{a_{1}}=\left(a_{x}, 0,0\right), \quad \overrightarrow{a_{2}}=\left(0, a_{y}, 0\right), \quad \overrightarrow{a_{3}}=(0,0, \zeta)
$$

Lattice constant $\mathrm{a}_{\mathrm{x}}$ has been predicted to range from 4.376 to $4.43 \AA[104,127]$ and $\mathrm{a}_{\mathrm{y}}$ ranges from 3.28 to $3.314 \AA[104,127]$. The volume of the unit cell is given by,

$$
V=\overrightarrow{a_{1}} \cdot \overrightarrow{a_{2}} \times \overrightarrow{a_{3}}=a_{x} a_{y} \zeta
$$

The reciprocal lattice vectors are,

$$
\begin{aligned}
& \overrightarrow{b_{1}}=2 \pi \frac{\overrightarrow{a_{2}} \times \overrightarrow{a_{3}}}{\overrightarrow{a_{1}} \cdot \overrightarrow{a_{2}} \times \overrightarrow{a_{3}}}=\frac{2 \pi}{a_{x}} \hat{\imath} \\
& \overrightarrow{b_{2}}=2 \pi \frac{\overrightarrow{a_{3}} \times \overrightarrow{a_{1}}}{\overrightarrow{a_{1}} \cdot \overrightarrow{a_{2}} \times \overrightarrow{a_{3}}}=\frac{2 \pi}{a_{y}} \hat{\jmath} \\
& \overrightarrow{b_{3}}=2 \pi \frac{\overrightarrow{a_{1}} \times \overrightarrow{a_{2}}}{\overrightarrow{a_{1}} \cdot \overrightarrow{a_{2}} \times \overrightarrow{a_{3}}}=\frac{2 \pi}{\zeta} \hat{k}
\end{aligned}
$$

The vectors $\hat{\imath}, \hat{\jmath}$, and $\hat{k}$ are unit vectors in the $\mathrm{x}, \mathrm{y}$, and $\mathrm{z}$ directions respectively.

Phosphorus atoms have an electron configuration of $3 \mathrm{~s}^{2} 3 \mathrm{p}^{3}$ for its valence shell. Each phosphorus atom bonds with 3 other neighboring phosphorus atoms to form its puckered hexagonal unit cell. Each of the three in-plane bonds are $\sigma$-bonds [128]. The 
valence-shell electron-pair repulsion theorem (VSEPR) [129] states that molecules minimize repulsion between valence shell electrons. This in turn factors into molecular geometry and is why each phosphorus atom has a lone pair of electrons. The lone pair electrons are in the $3 \mathrm{p}_{\mathrm{z}}$ orbital [128]. With respect to atomic plane of phosphorene, the $3 p_{z}$ orbital is directed perpendicular to the plane. Each $\sigma$-bond formed by the in-plane phosphorus atoms prevent those associated electrons from normal excitation into the conduction band. However, the lone pair electrons in $3 p_{z}$ orbital are less restricted. Phosphorene is different than graphene which has only one electron in the $2 \mathrm{p}_{\mathrm{x}}$ orbital that is nonbonding [130]. A phosphorus atoms lone electron pair, is also free to readily form covalent chemical bonds with other external atoms. The above may be one reason for phosphorene's lack of long term stability [131]. Four atoms in the unit cell of phosphorene result in 4 lone pairs (8 electrons) per unit cell available for conduction and bonding.

Density functional theory (DFT) has been used by scientist for over 50 years [121]. One of the most difficult problems in physics is determining properties of ground state many-body systems. All the information needed to determine those properties are found by solving the Schrödinger equation,

$$
\begin{gathered}
H \psi=E \psi \\
H=\sum_{i=1}^{N}-\frac{\hbar^{2}}{2 m_{i}} \nabla^{2}+V\left(r_{1}, r_{2}, \ldots, r_{N}\right)
\end{gathered}
$$

$\mathrm{H}$ is the Hamiltonian of the system, $\psi$ is the wave function, $\mathrm{E}$ is the energy, $\mathrm{V}$ is the potential and $\mathrm{N}$ is the number of elements. Analytical solutions to the Schrödinger 
equation can be done for problems such as hydrogen like atoms. Analytical solutions to many-body problems on the other hand, are very difficult and figuring it out would be a guaranteed Nobel Prize. Real world systems are many-body problems. As such the core of DFT, is a method for approximating solutions to many-body systems.

Unlike the $3 \mathrm{~N}(\mathrm{~N}=$ atoms $)$ variable wave-function assumption of the HartreeFock model, the Khon-Sham equations reduce the many-body problem by relating properties to the electron density [121]. The problem of non-interacting electrons simplifies the many-body problem. In the Schrödinger equation (eqn. 1.03), the potential term is replaced by an effective potential,

$$
V\left(r_{1}, r_{2}, \ldots \ldots, r_{N}\right)=V_{e f f}(r)=v(r)-e \phi(r)+v_{X C}(r)
$$

$v(r)$ is the external potential, $\varphi(r)$ is the electrostatic potential given by,

$$
\phi(r)=-e \int \frac{n\left(r^{\prime}\right)}{\left|r-r^{\prime}\right|}
$$

$\mathrm{n}\left(\mathrm{r}^{\prime}\right)$ is the electron density, $\mathrm{V}_{\mathrm{XC}}$ is the exchange-correlation potential defined as,

$$
v_{X C}(r)=\frac{\delta E_{X C}}{\delta n(r)}
$$

The electron interaction, is developed by modeling the exchange-correlation energy as a function of the electron density,

$$
E_{X C}=E_{X}+E_{C}
$$


Several approaches have been used to solve the exchange-correlation issue. The generalized gradient approximation and Perdew-Burke-Emzerhof (GGA-PBE) exchangecorrelation functional is one such approach [122]. Unlike the local density approximation (LDA), GGA does not require the electron density to be approximately homogenous. In addition, DFT with GGA does not require the atomic wave function to be localized on the atoms [122].

The Schrödinger equation is now written as,

$$
\left(-\frac{\hbar^{2}}{2 m} \nabla^{2}+v(r)-e \phi(r)+v_{X C}(r)\right) \psi_{i}=E \psi_{i}
$$

From Bloch's theorem, the wave function of an electron is written as,

$$
\psi=e^{i k * R} \psi_{t}(\vec{r}-\vec{R})
$$

variable $\vec{R}$ is the lattice vectors, $\psi_{t}=g_{1} \psi_{1}+g_{2} \psi_{2}+\cdots . .+g_{8} \psi_{8}$ for phosphorene. For the unit cell of phosphorene there are 8 electrons and their corresponding wave functions $\psi_{i}$. With i being intergers from one to eight. The constants $g_{1}$ satisfy the relationship,

$$
\left|g_{1}\right|^{2}+\left|g_{2}\right|^{2}+\cdots+\left|g_{8}\right|^{2}=1
$$

The Schrödinger equation can now be solved for the energy of the system. By writing the energy in terms of k-space (momentum) the energy dispersion relation can be determined. The direct band gap of phosphorene is found to be between 1 to $2 \mathrm{eV}$ at $\Gamma$ $[83,89,92,132]$. 


\subsection{SYNTHESIS OF TRANSISTION METAL DICHALCOGENIDES AND PHOSPHORENE}

Transition metal dichalcogenides (TMD) layers are similar to graphene. However, it is noted that a monolayer of TMD is actually a layer of transition metal atoms sandwiched between two layers of chalcogenides. Even with this difference, it was natural for the synthesis of monolayer TMD's to follow in the footsteps of graphene. Figure 1.5 shows the theoretically predicted monolayer TMD's [4]. Of these 44 combinations of TMD's, only 5 are predicted to be unstable.

To date there have been many monolayer TMD's synthesized. They include $\mathrm{MoS}_{2}, \mathrm{WS}_{2}, \mathrm{MoSe}_{2}$, and $\mathrm{WSe}_{2}$ [133-136]. However, many more have not yet been synthesized. There are several reasons why some monolayer TMD's have not been experimentally found. Theoretical calculations have shown that many TMD's have other stable crystalline structures [4]. If non-layered crystalline forms have similar or lower formation energies, the TMD's will prefer those structures due to systems preferring minimum energy configurations. Another reason why some TMD's have not been synthesized is due to the level of difficulty associated with developing proper synthesis techniques. If no recipe has been successfully created for a particular TMD, it could potentially take hundreds of experiments (the current works recipe for TMD's took over 100 experiments) to develop one. For chemical vapor deposition, variables such as proper volume flow rates, pre-cursor materials, temperature ramping rates, pressure, and many 
other factors can dictate the outcome of a recipe. Even if a recipe is known, variations in equipment such as heat loss through insulation can further complicate material synthesis.

\begin{tabular}{|c|c|c|c|c|c|c|c|c|}
\hline $\begin{array}{l}\mathbf{M}=\mathrm{Sc}, \mathrm{Ti}, \mathrm{V}, \mathrm{Cr} \\
\mathbf{X}=\mathrm{O}, \mathrm{S}, \mathrm{Se}, \mathrm{Te}\end{array}$ & \multicolumn{2}{|c|}{ Monolayer Stability } & \multicolumn{2}{|c|}{ Conductivity } & \multicolumn{2}{|c|}{$\begin{aligned} \mathbf{M} & =\mathrm{Mn}, \mathrm{Fe}, \mathrm{Co}, \mathrm{Ni} \\
\mathbf{X} & =\mathrm{O}, \mathrm{S}, \mathrm{Se}, \mathrm{Te}\end{aligned}$} & Monolayer Stability & Conductivity \\
\hline $\mathrm{ScO}_{2}$ & \multicolumn{2}{|l|}{ Stable } & \multicolumn{2}{|c|}{ Semiconductor } & \multicolumn{2}{|l|}{$\mathrm{MnO}_{2}$} & Stable & Metallic \\
\hline $\mathrm{ScS}_{2}$ & \multicolumn{2}{|l|}{ Stable } & \multicolumn{2}{|c|}{ Semiconductor } & \multicolumn{2}{|l|}{$\mathrm{MnS}_{2}$} & Stable & Metallic \\
\hline $\mathrm{ScSe}_{2}$ & \multicolumn{2}{|l|}{ Stable } & \multicolumn{2}{|c|}{ Semiconductor } & \multicolumn{2}{|l|}{$\mathrm{MnSe}_{2}$} & Stable & Metallic \\
\hline $\mathrm{ScTe}_{2}$ & Stable & & \multicolumn{2}{|c|}{ Metallic } & \multicolumn{2}{|l|}{$\mathrm{MnTe}_{2}$} & Stable & Metallic \\
\hline $\mathrm{TiO}_{2}$ & \multicolumn{2}{|l|}{ Unstable } & \multicolumn{2}{|l|}{ N/A } & \multicolumn{2}{|l|}{$\mathrm{FeO}_{2}$} & Stable & Metallic \\
\hline $\mathrm{TiS}_{2}$ & \multicolumn{2}{|l|}{ Stable } & \multicolumn{2}{|c|}{ Metallic } & \multicolumn{2}{|l|}{$\mathrm{FeS}_{2}$} & Stable & Metallic \\
\hline $\mathrm{TiSe}_{2}$ & \multicolumn{2}{|l|}{ Stable } & \multicolumn{2}{|c|}{ Metallic } & \multicolumn{2}{|l|}{$\mathrm{FeSe}_{2}$} & Stable & Metallic \\
\hline $\mathrm{TiTe}_{2}$ & \multicolumn{2}{|l|}{ Stable } & \multicolumn{2}{|c|}{ Metallic } & \multicolumn{2}{|l|}{$\mathrm{FeTe}_{2}$} & Stable & Metallic \\
\hline $\mathrm{VO}_{2}$ & \multicolumn{2}{|l|}{ Stable } & \multicolumn{2}{|c|}{ Metallic } & \multicolumn{2}{|l|}{$\mathrm{CoO}_{2}$} & Unstable & N/A \\
\hline $\mathrm{VS}_{2}$ & \multicolumn{2}{|l|}{ Stable } & Metallic & & $\mathrm{CoS}_{2}$ & & Unstable & N/A \\
\hline $\mathrm{VSe}_{2}$ & Stable & & Metallic & & $\mathrm{CoSe}_{2}$ & & Unstable & N/A \\
\hline $\mathrm{VTe}_{2}$ & Stable & & Metallic & & $\mathrm{CoTe}_{2}$ & & Stable & Metallic \\
\hline $\mathrm{CrO}_{2}$ & Stable & & Semicon & ictor & $\mathrm{NiO}_{2}$ & & Stable & Semiconductor \\
\hline $\mathrm{CrS}_{2}$ & Stable & & Semicon & ictor & $\mathrm{NiS}_{2}$ & & Stable & Metallic \\
\hline $\mathrm{CrSe}_{2}$ & Stable & & Semicon & ictor & $\mathrm{NiSe}_{2}$ & & Stable & Metallic \\
\hline $\mathrm{CrTe}_{2}$ & Stable & & Semicon & ictor & $\mathrm{NiTe}_{2}$ & & Stable & Metallic \\
\hline & & $\begin{array}{l}M=N b \\
X=O, S\end{array}$ & $\begin{array}{l}\text { Mo, W } \\
\text { Se, Te }\end{array}$ & Mon & r Stability & Conduct & ivity & \\
\hline & & $\mathrm{NbO}_{2}$ & & Unst & & N/A & & \\
\hline & & $\mathrm{NbS}_{2}$ & & Stabl & & Metallic & & \\
\hline & & $\mathrm{NbSe}_{2}$ & & Stabl & & Metallic & & \\
\hline & & $\mathrm{NbTe}_{2}$ & & Stabl & & Metallic & & \\
\hline & & $\mathrm{MoO}_{2}$ & & Stabl & & Semicor & ductor & \\
\hline & & $\mathrm{MoS}_{2}$ & & Stabl & & Semicor & ductor & \\
\hline & & $\mathrm{MoSe}_{2}$ & & Stabl & & Semicor & ductor & \\
\hline & & $\mathrm{MoTe}_{2}$ & & Stabl & & Semicor & ductor & \\
\hline & & $\mathrm{WO}_{2}$ & & Stabl & & Semicor & ductor & \\
\hline & & $\mathrm{WS}_{2}$ & & Stabl & & Semicor & ductor & \\
\hline & & $\mathrm{WSe}_{2}$ & & Stabl & & Semicor & ductor & \\
\hline & & $\mathrm{WTe}_{2}$ & & Stabl & & Semicor & iductor & \\
\hline
\end{tabular}

Figure 1.5 Stability and conductivity of several monolayer TMD's 


\subsubsection{Mechanical exfoliation}
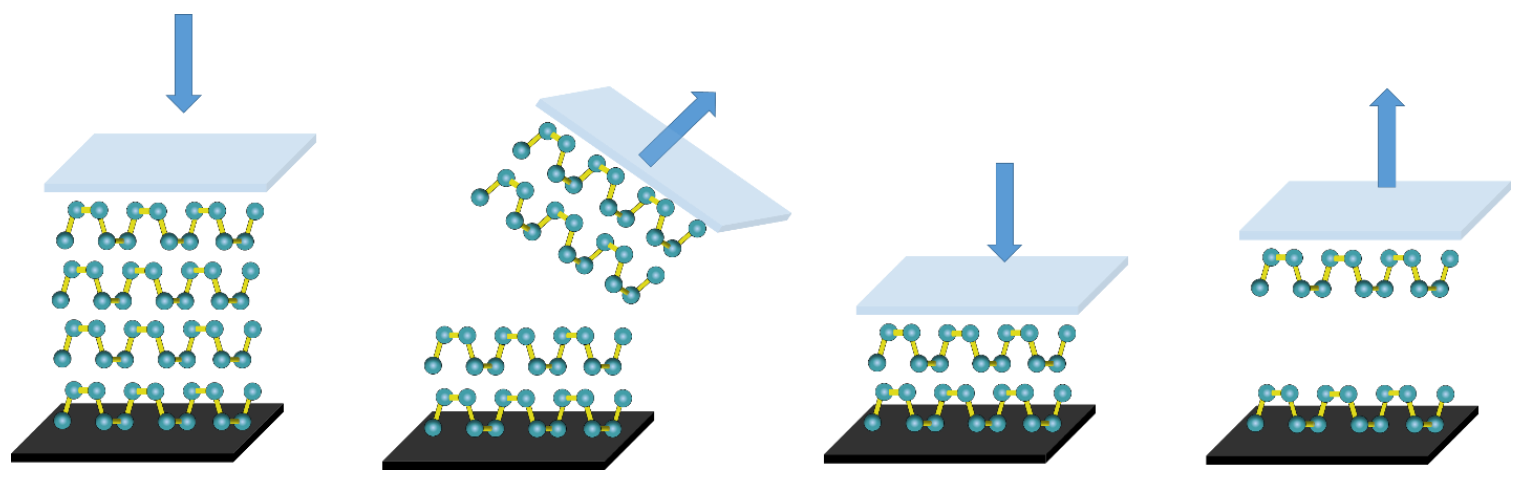

Figure 1.6

Schematic of mechanical exfoliation process

Mechanical exfoliation was the first method used to fabricate graphene [7].

TMD's and phosphorene followed suit. The drawback of mechanical exfoliation, is that it can only be used on materials that have a layered structure in bulk form. Fortunately, TMD's and black phosphorus have a layered structure even in bulk form. During mechanical exfoliation an adhesive backed tape can be used to remove layers of material $[7,24]$. This process is also known as the scotch tape method. Sizes of mechanically exfoliated material is relatively small but can be up $100 \mu \mathrm{m}$ [137] in lateral dimensions for best cases. Figure 1.6 shows a schematic of mechanical exfoliation. Of the other methods of synthesis, mechanical exfoliation produces the smallest yields. In addition, it is labor intensive. Although it has its drawbacks, mechanical exfoliation produces the highest quality single crystal monolayers. Crystal grain sizes depend on bulk material, which can be controlled with high precession thanks to advances developed for the electronics industry. In general the benefits of mechanical exfoliation is high mobility, grain boundaries are larger, and virtually any substrate can be used [138]. 


\subsubsection{Chemical Vapor Deposition}

\section{Chemical Vapor Deposition}

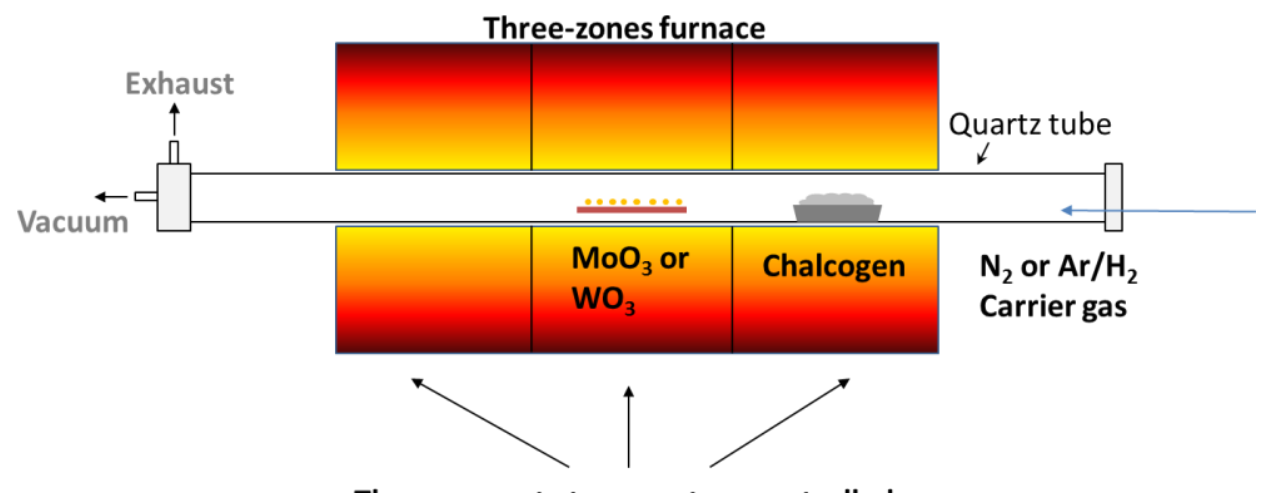

Three separate temperature controlled zones

Figure 1.7

Schematic of multi-zone quartz tube furnace used for CVD. This setup used in current work

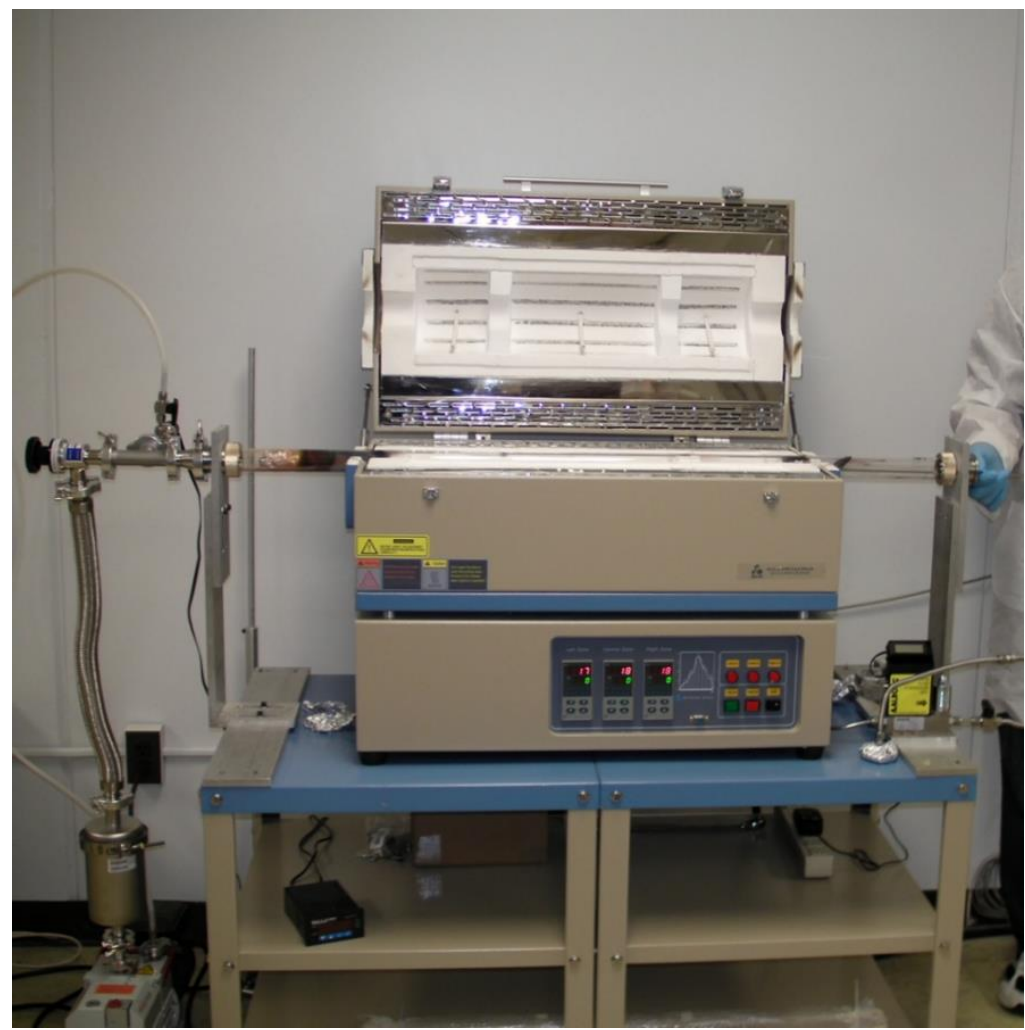

Figure 1.8

Multi-zone quartz tube furnace 
Chemical vapor deposition (CVD) is one of the few methods that can routinely reproduce monolayer TMD's. Figures 1.7 and 1.8, show how chemical vapor deposition works and the current works setup. CVD systems incorporate quartz tube furnaces, volume flow rate meters, pressure sensors, and vacuum systems. Quartz tube furnaces come in single and multi-zone heating stages.

CVD has several advantages over mechanical exfoliation. The main advantage is CVD has the ability to produce large scale monolayer films $[133,139,140]$. These films also have uniform thicknesses due to controlled growth parameters. Once a recipe has been developed, it can usually be repeated and successfully achieve monolayers. However, recipe development can be difficult. CVD can have over 10 parameters that can conceivable affect the growth of monolayers. Substrate preparation has a substantial impact on growth [141]. Arend M. van der Zande et al. found that improper cleaning of substrates decreases material yield and produces misshapen layers [141]. Lattice mismatch of substrate and TMD's can also affect the formation of monolayers. The elevated temperatures of CVD also impose restrictions on substrate selection. To date there has not been a reported CVD only synthesis of phosphorene.

\subsubsection{Liquid Mechanical Exfoliation}

There are several forms of liquid mechanical exfoliation (LME) that have been employed to synthesize monolayer TMD's and phosphorene. In order to decrease the inter-layer interaction in bulk material, lithium ions can be intercalated into the crystal lattice of TMD's [142, 143]. The lithium ions expand the interlayer spacing between 
successive layers and further weaken the van der Waals interaction. Lattice expansion of TMD's have been seen in lithium ion battery anodes [144, 145]. After lithium is used to expand the interlayer spacing, the material is suspended in a solvent. There are several studies that have found lithium insertion may be skipped [146, 147] when working with black phosphorus. This may be due to avoiding degradation of phosphorene.

Dimethylformamide and dimethyl sulfoxide have been found to be appropriate solvents for LME of phosphorene [147]. When lithium is used, deionized water is used as the solvent liquid [143]. The reaction between water and lithium follows the path $2 \mathrm{Li}(\mathrm{s})+$ $2 \mathrm{H}_{2} \mathrm{O}(\mathrm{l}) \rightarrow 2 \mathrm{LiOH}(\mathrm{aq})+\mathrm{H}_{2}(\mathrm{~g})$ [143]. Lithium hydroxide formation helps to consume any lithium present in the material. It also helps the exfoliation process since the lithium hydroxide bubbles also expand the lattice. However, the negatives outweigh the benefits in phosphorene due to water reacting with black phosphorus. The solvent/bulk material mixture is then sonicated for extended periods of time. Typical times for sonication of TMD's and phosphorene synthesis is over 15 hours [147]. Varying ultrasound frequency, duration of sample sonication, and temperature can alter the number of layers of the resulting suspension. Following sonication the samples are centrifuged. Centrifugation separates material based on number of layers and will be verified in subsequent sections of the current work. The final step of LME is to drop cast the suspension on a desired substrate.

LME has similar advantages to mechanical exfoliation in that virtually any substrate can be used. However, LME suffers from less control over the number of layers due to flake restacking [146]. Employing spin coating can improve uniformity of layering number in samples. Also the control of exact monolayers thorough LME parameters is 
more difficult. The results obtained for a particular set of LME parameters causes a range of layering numbers to be produced as opposed to a specific amount of layers.

\subsection{Modifications of Transition Metal Dichalcogenides and Phosphorene}

As two-dimensional materials mature, more progress is made in altering their inherent properties. Heterostructures, doping and surface functionalization have been the methods employed to tailor properties of semiconductors. For 2D materials to be of further use in advanced electronics, the understanding of their properties and how they can be enhanced or quenched is of upmost importance. Graphene is a prime example. By functionalization of graphene using nitrogen in RF plasma, a metal-insulator transition was found in graphene [148]. Heterostructures of graphene have been synthesized to take advantage of its long range transport and massless Dirac fermions but overcome its zero band gap [137, 149]. Modifying graphene in order to overcome its deficiencies and tailor its properties was a natural progression. The same progression has occurred in TMD's and phosphorene.

\subsubsection{Heterostructures}

In regards to layered systems, there are two types of heterostructures. Stacked heterostructures involve the successive vertical stacking of different $2 \mathrm{D}$ materials. Inplane heterostructures are formed by bonding dissimilar compounds within individual planes. Stacked structures can be made by utilizing combinations of synthesis methods and the subsequent use of transfer techniques $[43,150]$. Chemical etching of substrates 
can be used to separate the substrate from the monolayer film [151]. The newly freed film can then be transferred on top of other monolayer films forming stacked heterostructures. Chemical etching, electrostatic transfer, mechanical exfoliation, and chemical vapor deposition have all been used to produce stacked heterostructures [40]. In-plane heterostructures have proven to be much more difficult to synthesize [39]. Difficulties arises from chemical bonding the in-plane atoms, which is not an issue for stacked structures that only interact through van der Waals. Chemical vapor deposition has been the method of choice in synthesizing in-plane connections. By utilizing the different melting points of $\mathrm{MoS}_{2}$ and $\mathrm{WS}_{2}$, it was shown that systems comprised of both stacked and in-plane heterostructures could be synthesized from monolayer films [39].

The purpose of forming heterostructures is due to the combined systems having unique properties that may differ from the separated individual components. One such theoretical study found that by stacking TMD's with the same transition metal but different chalcogen atoms the systems can retain the direct band gap electronic structure that is only seen in monolayer TMD's [63]. This is in contrast to the stacking or homostructures that TMD's naturally form for several layers of the same TMD. Multiply stacking of the same TMD's results in the transition from direct to indirect band gap [13]. Direct band gaps have advantages of being more efficient in their use of photons. Transitions in direct band gaps do not require the momentum from phonons unlike indirect band gap transitions. Other benefits include the band alignments of the individual materials. As the different systems in a heterostructure are brought in contact the conduction band minimum (CBM) and valence band maximum (VBM) of each material adjust until equilibrium is reached. In reality charges are exchanged between the 
materials as their respective fermi levels reach their equilibrium point. In turn the CBM of the combined system may actually lie within one material while the VBM may lie in the other material [63]. As an electron is excited and a hole is generated in the above system, they become electronically distanced. However, due to the layer separation of the CBM and VBM, the electron and holes are also physically separated. This separation may increase exciton (electron-hole pairs) lifetimes which is useful in photocatalysis, solar cells, photodetectors, and other junction devices.

\subsubsection{Doping}

Incorporating atoms directly into the lattice of another material in small quantities is considered to be doping. The difference in doping and alloying is one of material concentration. In alloyed systems, the individual elements are in similar concentrations. Dopant atoms can increase the amount of electrons in a 2D material. Each atom in a $\mathrm{MoS}_{2}$ layered system has active sites along its edges [152]. Adding atoms into TMD's that have more electrons than can bond to the TMD increases charge carriers. After bonds are formed, the dopant atom still contributes electrons to the system. This type of dopant is called an n-type dopant or donor. The energy level of the dopant electrons is usually at levels near the CBM of the original systems (shallow level). Finite temperatures can supply enough energy to excite these donated electrons into the CBM where they are free to contribute to conduction of the 2D system. A similar statement can be had for dopant atoms that have less electrons. These type of dopants, are p-type (acceptors) and supply holes into the VBM of the system. The added conduction carriers increase the conductivity of the 2D system. As more and more dopants are added, the fermi level of 
the original system shifts towards the position of the dopant atoms. It was found that TMD's doped with potassium doping at the drain/source contacts in an FET creates low contact resistance [153].

There are many techniques for doping 2D materials. Using a very precise control of precursor material in CVD, it has been shown that graphene can be doped with nitrogen [154]. Plasma assisted CVD [148] and chemisorption [155] have similar characteristic . In order to introduce atoms in to the lattice of a system, one can increase the energy of the dopant atoms. Plasma assisted doping can cover large area samples. Plasma techniques also have the potential to degrade the sample.

When a dopant atom is introduced into a system it can occupy a lattice site that would have otherwise contained an atom from the original system. Taking over a lattice site by a dopant is called substitutional doping. On the other hand, dopant atoms can occupy space in between the lattice sites of a crystalline material and is called interstitial doping. If the interstitial dopant atoms are too large they can cause damage to the crystal structure of the system and destabilize it. This can also happen for substitutional dopants. There is a trade off with increasing conductivity through doping and decreasing charge carrier mobility. For dopant atoms that do not have atomic radii close to their host systems, charge carriers can see the dopant atoms as defect sites. In addition, lone pairs of electrons in the host system due to p-type dopants can induce magnetic moments [156, 157]. This effect can be beneficial in 2D based spintronic devices, however for other devices this may be detrimental. The final issue that dopant atoms can have is that they can be rejected from the host material if they exceed a certain amount. Which may be considered a form of precipitation. 


\subsubsection{Surface/Edge functionalization}

Gas molecule interaction with 2D materials is of great importance. Due to dangling bonds on edges and lone electron pairs atoms can readily be absorbed onto $2 \mathrm{D}$ systems. Functionalization can be defined as attaching elements to the 2D systems surface and/or edges. Unlike doping, the atoms do not have to incorporate into the crystal structure of the sample. Functionalization can be done much simpler than doping as direct exposure to various gases can induce atom attachment [158]. J. P. Li et al. found that functionalization could be enhanced by using lasers [159]. Controlling laser power was shown to directly control doping in ambient conditions. $\mathrm{WS}_{2}$ nano-flakes have been shown to have gas sensing capabilities through surface physical-absorption of $\mathrm{NH}_{3}$ [160]. Due to phosphorus atoms having a lone pair, it has been found that various atoms can be easily bonded to phosphorene. Several theoretical studies have shown that oxygen is one of the most favorable atoms to bond to phosphorene [157, 161].

Besides its simplicity, functionalization has another important role. As different atoms are attached to the 2D systems, various properties can change. The band gap of TMD's and phosphorene can be varied over large ranges. Being able to engineer band gaps can allow novel optical devices to be fabricated. TMD's have the added benefit of having over 44 types of TMD's even without functionalization. The number of combinations of functionalization possibilities for TMD's is extremely vast. Realization of commercial products such as photodetectors and gas sensors by surface functionalization may be the next big leap in optoelectronics. 


\subsection{Phosphorene anisotropy}

It is evident that phosphorene has taken the research community captive just as graphene has. With over 60 publications per month in 2015, the rate of increase in phosphorene research is growing. One of the main contributors to the interest in phosphorene is its remarkable anisotropic properties [94, 106-113, 162-169].

Two-dimensional materials exhibit inplane and out of plane anisotropy due to differences in bonding. Inplane bonding is dominated by covalent/ionic interactions and out of plane is van der Waals dependent. Vertical conductivity through one layer into

another layer is dominated by quantum tunneling. As such it is much lower than in plane conductivity. Charge carrier motilities vary for in plane versus out of plane for graphene. However, for phosphorene there is an added anisotropy in the zigzag and armchair directions. Electric polarizability has a large difference in values for the zigzag direction and armchair direction [149]. By orientating the zigzag direction in the same sense as charge carrier flow in the conduction channel in a field effect transistor, device performance can be maximized [97]. Electronic transport is not the only property that is effected by anisotropy in phosphorene. Ion diffusion is of extreme importance in secondary battery anodes and cathodes. For anodes, it is extremely important to have high diffusion rates [170-172]. Due to the topological structure of phosphorene, anisotropic diffusion in the zigzag and armchair directions is difficult to determine and current theoretical models show conflicting results [164, 170-176]. The anisotropy in phosphorene can be utilized to make novel batteries and optoelectronic devices. 


\section{CHAPTER 2 - SYNTHESIS AND CHARACTERIZATION OF TRANSITION METAL DICHALCOGENIDES AND PHOSPHORENE}

\subsection{Introduction}

As outlined in section 1.5 , there are many ways in which 2D systems can be fabricated. Many factors come into play when developing techniques for synthesis. For TMD's, the growth and characterization of monolayer films has been well documented [133, 177-180]. Monolayers have many properties, however controlled engineering of those properties is vital. Heterostructures are one route to do the above. Research into stacked heterostructures has progressed, however due to synthesis difficulties, in-plane heterostructures have progressed at a much slower rate. As mentioned early, both inplane and stacked heterostructures of TMD's have been previously reported [39]. However, the above process had no control over the formation location of these heterostructures due to using an all CVD method. The need for having more control and reproducibility is of extreme importance, especially in scaling production of real world devices. As such, the current work has combined the control of Thermal Evaporation with CVD in order to create novel TMD heterostructures with precise patterned in-plane and stacked heterostructures. The difficulties involved in synthesis of phosphorene are well known. Due to the relative instability of black phosphorus in ambient conditions and extreme conditions required to create bulk material. As such, fundamental research in phosphorene is of more importance. The synthesis of bulk black phosphorus used a short 
transport closed method [80] for the current work. Major factors in the choice of synthesis technique of phosphorene include prevention of degradation and speed of which devices could be fabricated. With the above in mind, liquid mechanical exfoliation of bulk black phosphorene is employed for the synthesis of monolayer phosphorene [146, $147,181,182]$.

\subsection{Thermal evaporation and CVD Kinetics and transport}

Thermal evaporation has been used for many years in the fabrication of materials. An illustration of the thermal evaporation process and equipment used is shown in figure 2.1. In thermal evaporation, precursor material is evaporated using resistive heating of a boat (holder) and travels towards a substrate situated above the initial precursor material. As the atoms come into contact with the substrate, they adhere to the substrate. One of the benefits of thermal evaporation is the high level of control of the amount of material being deposited on the substrate. Also, large area synthesis is possible. In large scale production however, thermal evaporation can become inefficient if direction mechanisms are not used due to wasted deposition outside of the substrate. Thermal evaporation has three main components. These components involve the vacuum chamber environment, source evaporation/sublimation, and substrate adhesion. 
a

Thermal Evaporation
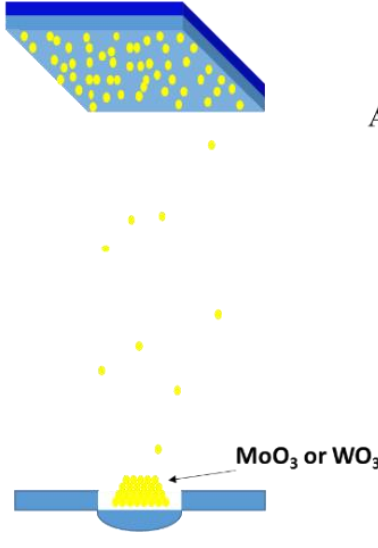

Autotransformer

d

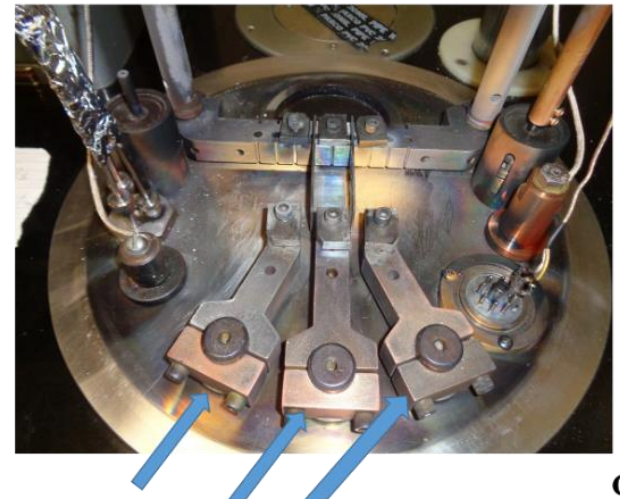

Electrodes that connect to boats $\mathrm{b}$
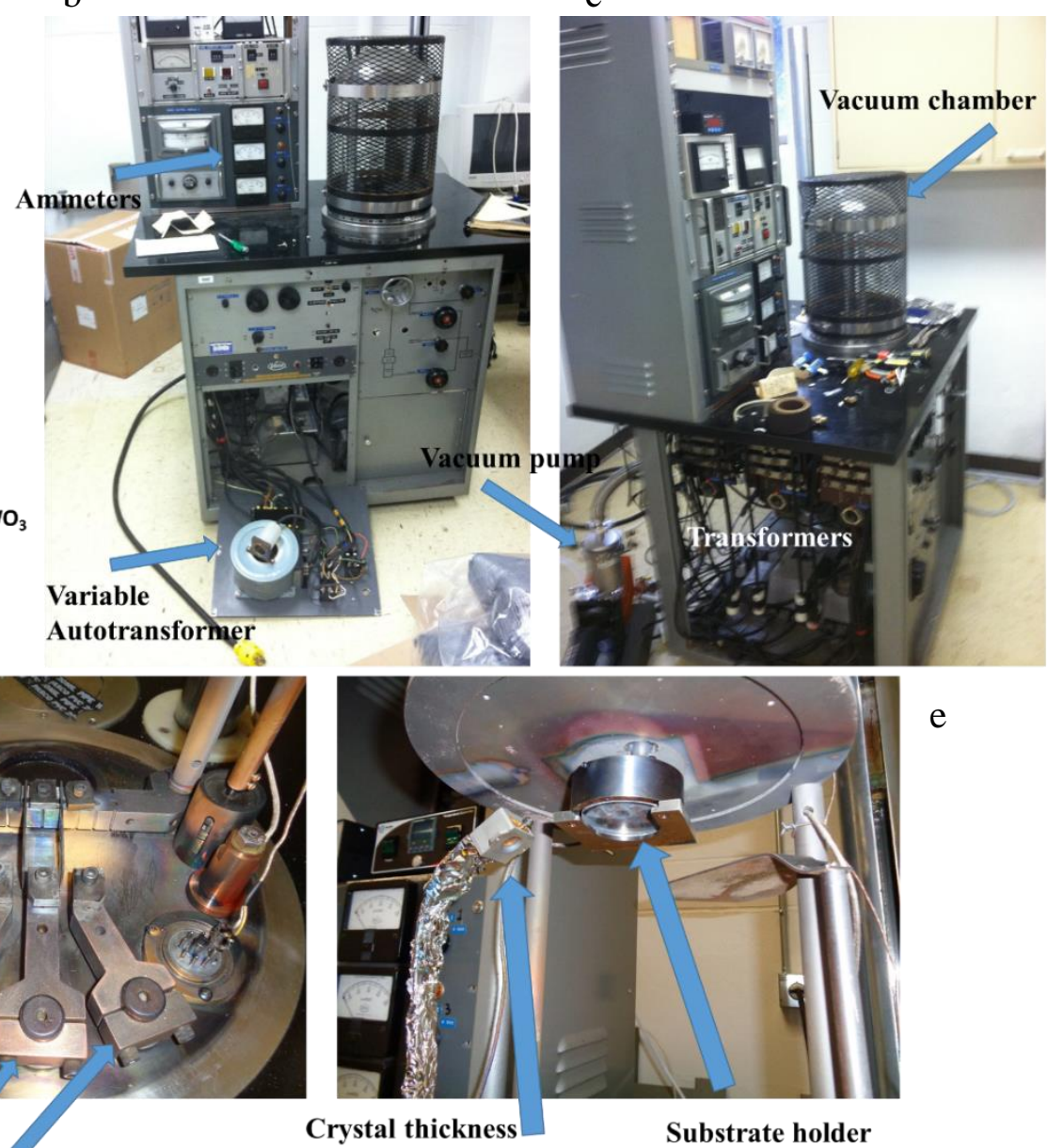

sensor

Figure 2.1

a.) Schematic of thermal evaporation process. b.) and c.) Refurbished thermal evaporator. d.) and e.) Inside vacuum chamber.

In figure $2.1 \mathrm{c}$, the vacuum chamber is shown. It is also called the bell jar. This is the area in which the source material will be evaporated. It contains all the electronic measuring components, conductive sample holders (boats), substrates, and vacuum ports. The bell jar is usually constructed from Pyrex or quartz. It can also be shielded to prevent high energy UV radiation from harming the user. Figures $2.1 \mathrm{~d}$ and e show the inside of the bell jar. The thermal evaporator used in the current work had a tri-source setup. Multiple precursors could be evaporated without having to break vacuum. 
Bell jars also serve the purpose of providing a surface to seal and hold vacuum. Thermal evaporation was done at $10^{-5}$ Torr for the current work. Conducting evaporation in vacuum has several benefits. It decreases potential contamination caused by impurity atoms and lowers the temperature needed to evaporate the source materials. Heat transfer from the evaporating source to the substrate is also decreased. To illustrate this difference, thermodynamics is employed. The first law of thermodynamics states that for all adiabatic processes between two specified states of a closed system, the net work done is the same regardless of the nature of the closed system and the process. The above can be restated as the conservation of energy principle,

$$
E_{\text {in }}-E_{\text {out }}=\Delta E_{\text {system }}
$$
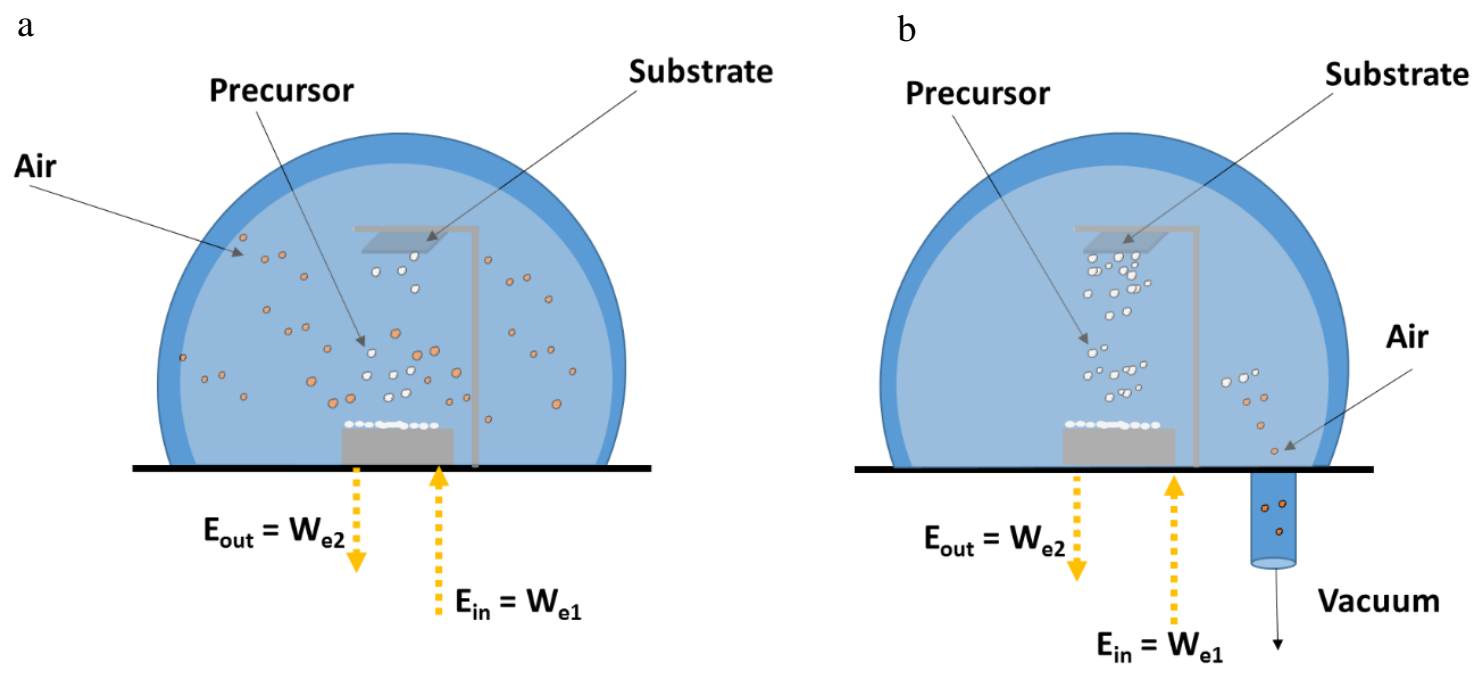

Figure 2.2 a.) Schematic of thermal evaporation done at standard atmospheric pressures. b.) Schematic of thermal evaporation done at low pressures. 
Figure 2.2 shows the bell jar for two different situations. If the bell jar is assumed to be a closed system, figure 2.2 a represents this. This assumption can be valid if the vacuum pumping system is off and the system has negligible leaking. The other situation is of the bell jar with the vacuum pumping system turned on and it also is assumed that mass entering/leaving the system has reached an equilibrium state due to limitations of vacuum seals and pumping systems. As such this can also be considered a closed system. Focusing on the energy that is leaving the boat, there are three forms of heat transfer. Conduction, convection, and radiation. Conduction is due to the direct transfer of energy from more energetic atoms to less energetic atoms through contact. For the low pressure system this is negligible because contact from adjacent atoms happens less frequently. Rate of heat conduction is given by,

$$
Q_{\text {cond }}^{\cdot}=k_{t} A \frac{\Delta T}{\Delta x}
$$

$Q_{\text {cond }}$ is the time dependent heat conduction in watts, $\mathrm{k}_{\mathrm{t}}$ is thermal conductivity of the material, $\mathrm{A}$ is the area normal to the direction of heat transfer, $\Delta \mathrm{T}$ is the change in temperature from point $\mathrm{x}_{1}$ to point $\mathrm{x}_{2}$. For this analysis, $\mathrm{x}_{1}$ is the boat, $\mathrm{x}_{2}$ is the substrate, and $\mathrm{k}_{\mathrm{t}}=0.026 \mathrm{~W} / \mathrm{m} * \mathrm{~K}$ for air. Convection is assumed to be zero for both systems since it is due to energy transfer from a solid and adjacent flowing fluid. The bell jar's solid surface is far enough away from the boat and substrate so that it is not a factor. In an attempt to understand the continuous range of light emitted from solid objects, J. Stefan in 1879 experimentally related the temperature of a solid object to the power it emits as light. The Stefan-Boltzmann law,

$$
Q_{\text {rad_max }}=\sigma A T_{S}^{4}
$$


$\mathrm{T}_{\mathrm{s}}$ is the absolute temperature of the solid, $\mathrm{A}$ is the area, $\sigma=5.67 * 10^{-8} \mathrm{~W} / \mathrm{m}_{2}$ is the Stefan-Boltzmann constant. $Q_{\text {rad_max }}$ is the maximum rate of radiation emitted. This is only vaild for blackbody structures. Real world solids have an additional multiplicative constant $\varepsilon$, which is the emissivity of the surface. The Stefan-Boltzmann law is now,

$$
Q_{\text {rad }}^{\cdot}=\varepsilon \sigma A T_{s}^{4}
$$

Looking at the two systems, it is evident that the substrate that is not under vacuum will receive more energy. This is due to it receiving energy from the boat due to conduction and radiation. The substrate in vacuum, only receives radiation. Vacuum is also essential for allowing evaporated source atoms to actually reach the substrate. In figure 2.2a there are more molecules in the bell jar which collide with the evaporated precursors. The mean free path is defined as the average distance an element travels between collisions,

$$
\lambda=\frac{k T}{\sqrt{2} \pi P d^{2}}
$$

$\lambda$ is the mean free path, $\mathrm{k}$ is the Boltzmann constant, $\mathrm{T}$ is temperature, $\mathrm{P}$ is pressure and $\mathrm{d}$ is molecular diameter of the atoms. It is noted that this equation for mean free path does not take into account collisions with atoms of different molecular diameters. Pressure is inversely proportional to mean free path. By lowering pressure the mean free path can be increased. At pressures of $10^{-5}$ Torr the mean free path is over $500 \mathrm{~cm}$. Typical distances from source to substrate are under $50 \mathrm{~cm}$. The above means that evaporated atoms follow a straight path with respect to where they started. In order to reach these pressure levels care must be taken in the design of the thermal evaporation system. Once $10^{-3}$ Torr 
pressures are reached, most roughing pumps are no longer effective. The reason is due to the increase in mean free path. It becomes less likely that gas molecules will come into contact with the rotating mechanisms of the roughing pump which pull material down. To further pull down the pressures momentum transfer or ionization pumping techniques must be used. Turbomolecular pumps transfer momentum from rotating blades transferring momentum to gas molecules. The direction of the transfer forces the molecules to leave the vacuum system. Oil diffusion pumps have a similar effect, except instead of using rotating blades, it uses oil to transfer momentum to the gas molecules. This shows the importance of vacuum in thermal evaporation.

Selection of precursor material involves the balancing chamber conditions. Those properties include lowering evaporation temperatures by decreasing pressure and insuring the chemical composition of the precursor is the desired one when it hits the substrate. Phase diagrams are extremely important in controlling composition. Materials have a preference on their chemical makeup that is dependent on pressure and temperature. The correct pressure must be selected to prevent precursors from undergoing a composition change. Such as $\mathrm{MoO}_{3}$ losing oxygen during the evaporation process and arriving at the substrate as Mo. In addition, higher melting point precursors such as tungsten will emit UV radiation due to the high energies need to evaporate them. For high melting point materials, electron beam evaporation is recommended. Precursor materials come in pellets, powders, and filaments. Powders usually require less energy to evaporate, and have a more uniform gas distribution in the vacuum chamber.

One of the most important components of thermal evaporation, is the monitoring process of how much material is being deposited on the substrate. The simplest method 
of determining material deposition amounts (which are directly related to the number of layers in a 2D material), is to develop a calibration based on time spent at the evaporation temperature. For a particular evaporation time and ex-situ thickness study such as atomic force microscopy, one can generate a relationship from the time spent evaporating material, to the thickness of the deposited material. This technique is simple and does not require adding electronics in the deposition chamber of the thermal evaporation system. However, this approach is prone to inaccuracies due to errors in timing evaporation periods. As such, this approach is used as a calibration for new systems and troubleshooting to insure other techniques for monitoring material deposition are working properly. Another method employs the use of a crystal thickness sensor. Crystal thickness sensors operate on the concept that resonant vibrating frequency of a crystal changes as material is deposited on it. Crystal sensors must be replaced regularly. Each sensor's vibrational calibration with respect to material deposited is only valid for a certain amount of deposited material. Sensors should be placed at the same level as the substrate to ensure proper reading. If it is at a different height, calibrations must be made to ensure film thickness monitoring. It is important to verify the reading of the crystal sensor by other methods such as the aforementioned ex-situ technique.

Precursor evaporation is done by converting electrical energy into heat. The power coming into the boat is,

$$
\dot{W}_{e}=V I
$$

with V and I being potential difference and current respectively. Knudsen [183] found that the maximum number of molecules evaporating as a function of time for a given area is given by, 


$$
\frac{d N_{e}}{A d t}=P_{e}(2 \pi m k T)^{-1 / 2}
$$

$\mathrm{N}_{\mathrm{e}}$ is the number of molecules, $\mathrm{A}$ is the surface area, $\mathrm{m}$ is the molecule mass, $\mathrm{k}$ is the Boltzmann constant, $\mathrm{P}_{\mathrm{e}}$ is the partial pressure (pressure exerted by the evaporating atoms on the other atoms in the system), and $\mathrm{T}$ is the temperature. Integrating equation 2.06 with steady state constant temperature and pressure gives the number of molecules evaporated for a certain time period $\Delta \mathrm{t}$,

$$
N_{e}=A P_{e}(2 \pi m k T)^{-1 / 2} \Delta t
$$

As the atoms come into contact with the substrate, the excess energy they gained during the evaporation process is transferred to the substrate. The number of atoms that reach a particular distance $\mathrm{Y}$ is determined by the following,

$$
N=N_{0} \exp \left(\frac{-Y}{\lambda}\right)
$$

$\mathrm{N}_{0}$ is the total number of initial atoms trying to travel the distance Y. Also $\lambda$ is the mean free path. From equation 2.07, the total number of initial atoms in equation 2.08 is equal to the number of evaporated atoms.

$$
N_{0}=N_{e}
$$

Combing equation 2.07 and 2.08 gives the number of atoms that reach a distance of $\mathrm{Y}$, in terms of pressure, temperature, and mean free path,

$$
N=A P_{e}(2 \pi m k T)^{-1 / 2} \Delta t \exp \left(\frac{-Y}{\lambda}\right)
$$


The mean free path was defined in equation 2.04. Adding this into equation 2.10 gives,

$$
N=A P_{e}(2 \pi m k T)^{-1 / 2} \Delta t \frac{1}{\exp \left(\frac{\sqrt{2} \pi P d^{2} Y}{k T}\right)}
$$

Some of the factors that directly control deposition rates in thermal evaporation are shown in equation 2.11. As expected increasing duration of deposition increases the number of atoms that reach the substrate. Increasing pressure has an inverse relationship with mean free path. Increasing pressure in equation 2.11 decreases the number of atoms that traverse the distance $\mathrm{Y}$ due to a decrease in mean free path.

Simply getting to the substrate is not enough for atoms. To form films, the atoms must adhere to the substrate. The excess energy the atoms have, is transferred to the substrate. Several mechanisms are present that allow for the atoms to adhere to the substrate. Mechanical adhesion forms when atoms are trapped on the surface of the substrate due to surface irregularities. This can be done by cavities on the substrate surface formed by photolithography or etching. Another adhesion mechanism is due to electrostatic interaction. This is due to the van der Waals nature of the layered materials. In addition to adhering to the substrate, the atoms may diffuse across the surface of the substrate. This effect is only pronounced if the substrate is heated, which supplies the deposited atoms with more energy.

As shown in figure 1.7, chemical vapor deposition (CVD) involves transportation of vapor phase chemicals, temperature control, and removal of unused precursors and by products. Transport throughout the reactor can be turbulent or laminar, varying with location in the chamber. Flow profiles also depend on carrier gas volume flow rates. 
Higher flow rates increase turbulent flow and is harder to reproduce results. One of the first issues to address in CVD reactors is to determine the nature of fluid dynamics present. When the mean free path of atoms in the furnace is much lower than the dimensions of the furnace, the flow of material can be viewed as continuous. The Knudsen number is a ratio of the chamber size (quartz tube diameter, length, or some other characteristic component variable L) to the mean free path. When the mean free path is comparable to the dimensions of the furnace, the flow is no longer continuous. The atoms have comparable probability of impacting the chamber walls as colliding with other atoms. Continuous approximations are valid when the Knudsen number is much larger than 1 and discontinuous when it is less than one.

Transport of material across an area $\mathrm{A}$ and through a volume $\mathrm{V}$ is defined as the flux,

$$
\vec{J}_{c}=n \vec{u}
$$

with $\mathrm{n}=$ molecular density and $\overrightarrow{\boldsymbol{u}}=$ velocity. If the velocity is assumed to be the overall velocity of the gas flow, this type of flux is associated with convection. Equation 2.12 does not take into account diffusion, which is also responsible for mass transport. Diffusion is due to thermal motion of molecules. As this motion is normally random, there usually does not exist a net motion of material. In the case of varying gradients, such as temperature and material concentration, there will be net motion in a parallel direction of decreasing gradient with $\mathrm{D}$ being the diffusion constant, 


$$
\begin{aligned}
& \vec{J}_{d x}=-D \frac{d n}{d x} \hat{\imath} \\
& \vec{J}_{d y}=-D \frac{d n}{d y} \hat{\jmath} \\
& \vec{J}_{d z}=-D \frac{d n}{d z} \hat{k}
\end{aligned}
$$

The above diffusive flux dependent equations are valid for the continuous flow model. For discontinuous flow models, the diffusion constant can be approximated as the Knudsen diffusivity, with $\mathrm{c}$ being the mean thermal velocity of the molecules

$$
D(\text { Knudsen })=c L / 3
$$

Time dependence of diffusion is given by the following partial differential equations,

$$
\frac{\delta n}{\delta t}=D \nabla^{2} n
$$

$\nabla^{2}$ is the Laplacian operator,

$$
\nabla^{2}=\frac{\delta^{2}}{\delta x^{2}}+\frac{\delta^{2}}{\delta y^{2}}+\frac{\delta^{2}}{\delta z}
$$

For CVD reactions, flux due to diffusion and convection should both be accounted for, to insure the proper ratios of precursor materials are being supplied at the appropriate times and locations within the system. It is noted that diffusion dominates on the surface of the substrate due to an interface layer on top of the substrate. 


\subsection{Synthesis of Transition Metal Dichalcogenides Heterostructures}

Recipes for the growth of TMD monolayers vary considerable. Some operate at low pressures, however atmospheric synthesis is idea for simplicity. Temperature ramping rates, maximum temperatures, volume flow rates, and carrier gases all vary from recipe to recipe. However, the one thing they have in common is a lack of control over the locations on the substrate in which the material is grown. In order to create intricate patterns with an all CVD method, specialized materials are needed. CVD of TMD's usually involve temperatures from 600 to $1000{ }^{\circ} \mathrm{C}$. These elevated temperatures make the use of typical masking material not an option. Other options include ceramic mask or etching the substrate in particular directions to make artificial nucleation sites. Both techniques require the use of specialized equipment. Being able to accurately control the formation of materials is paramount when developing advanced optoelectronic devices. A novel method has been designed in the current work to produce TMD based heterostructures. The method allows patterned heterostructures without the need for expensive photolithographic techniques.

Before any deposition can take place substrates were selected. The current work chose $\mathrm{SiO}_{2} / \mathrm{Si}$ substrates. Silicon dioxide has a melting point over $1,600{ }^{\circ} \mathrm{C}$ at atmospheric conditions. Silicon has a melting point over $1,400{ }^{\circ} \mathrm{C}$ at atmospheric conditions also. The temperature ranges that will be undertaken during material synthesis is well below these points. For TMD synthesis, substrate lateral dimensions were 10 by $10 \mathrm{~cm}$, with an oxide thickness of $300 \mathrm{~nm}$. Growth was also done on other substrates such as sapphire and quartz. 
Proper cleaning of substrates has been shown to dramatically change results of CVD experiments. As such, the $\mathrm{SiO}_{2} / \mathrm{Si}$ substrates were washed with acetone and ethanol, followed by ultra-high purity compressed nitrogen blow drying. Ethanol is used to remove residual acetone. Without the ethanol wash, deposits could be visibly seen forming on the substrate as the acetone dried. The nitrogen blow drying is used to further ensure both acetone and ethanol are removed from the substrate. It is imperative that as much organic matter as possible is removed from the substrate prior to cleaning with piranha solution. Piranha solution removes residual organic matter. Exposing piranha solution to bulk organic matter can result in explosions. Piranha solution was prepared with a 3:1 ratio of $\mathrm{H}_{2} \mathrm{SO}_{4}$ to $\mathrm{H}_{2} \mathrm{O}_{2}$. After the hydrogen peroxide was slowly added to the sulfuric acid, the mixture was allowed to cool down. The substrates were slowly added to the piranha solution and stayed submerged for 40 minutes. Following piranha solution, the substrates were washed several times with fresh deionized water. The final step involved one more wash in ethanol followed by a final nitrogen blow dry. Piranha solution was chosen to wash the substrates because it removes organic matter from the substrates and improves growth of films. 
(1) $\mathrm{MoO}_{3}$ or $\mathrm{WO}_{3}$ Thermal Evaporation

(2) Mask removal

(2B) Chalcogenization

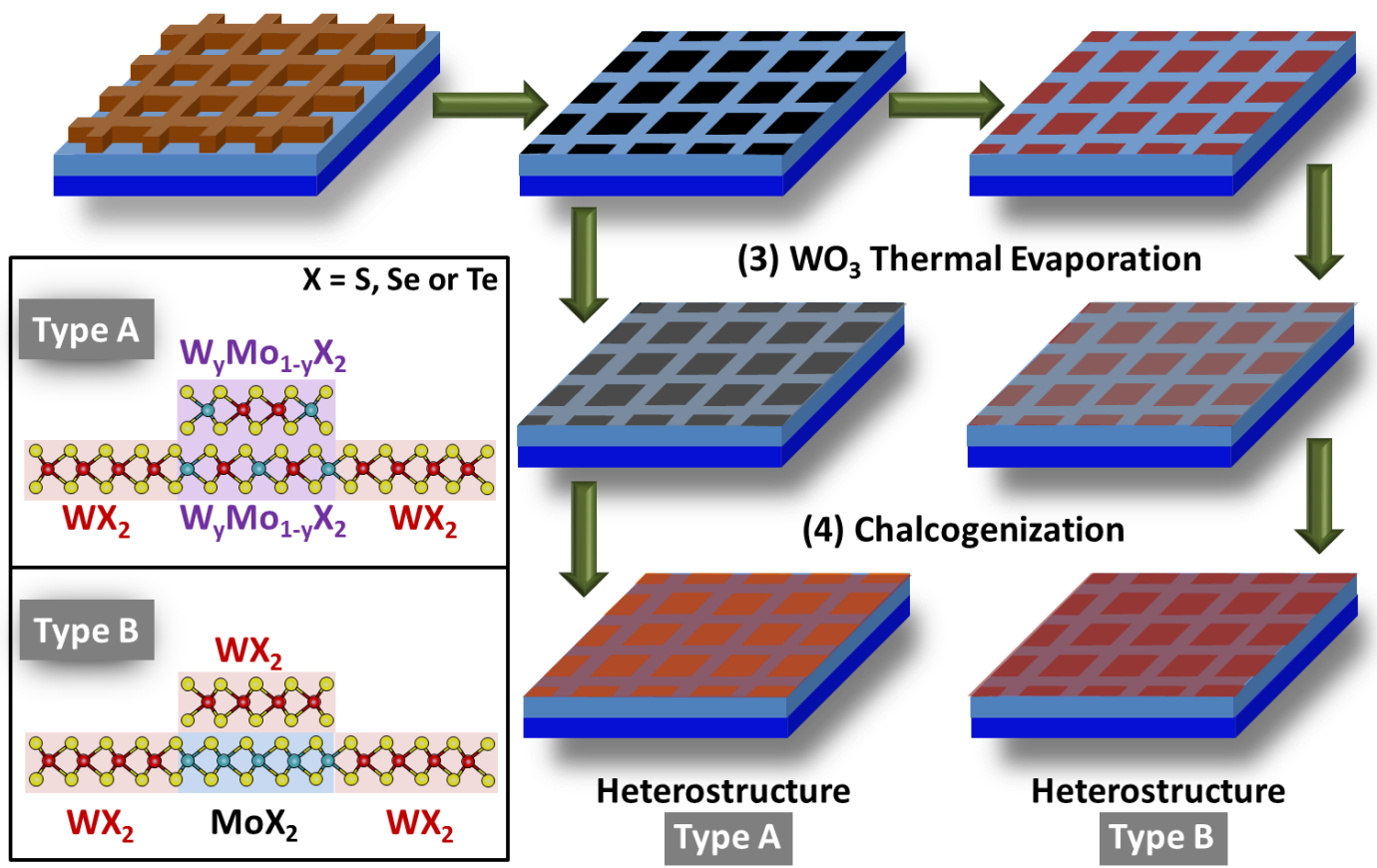

Figure 2.3 Schematic of growth process for heterostructures made by series A and series B methods. Inset is of predicted structures formed after synthesis.

Directly after cleaning the substrates, they are transferred to the thermal evaporator and put under vacuum to prevent surface contamination. Schematic for film growth is shown in figure 2.3. Methods for the growth of TMD heterostructures are designated as Series A and Series B. In order to synthesize series A heterostructures, a grid is first placed directly on the substrate before it is placed inside the thermal evaporator. The grids used for this study were readily available standard copper transmission electron microscopy (TEM) grids. By using standard grids, the cost of this process is greatly reduced. To further improve the contact between the grids and substrates, a custom built substrate holder was used. The holder allows for TEM grids to be held in place by the weight of the substrate pressing down upon it. Following 
placement of the substrate on the grid, the pressure is decreased to $10^{-5}$ Torr. It was found that increasing or decreasing the pressure beyond $10^{-5}$ Torr prevented reproducible outcomes of the deposition process. Once appropriate pressures were reached, precursor material was deposited on to the substrate through the grids. The precursor material was powder form of $\mathrm{MoO}_{3}$ or $\mathrm{WO}_{3}$, depending on desired composition of finial heterostructure. About $8 \AA$ of deposition material as determined from the crystal thickness sensor, correlated to the subsequent formation of one monolayer TMD. In addition the thermal evaporator had to be continually calibrated due to deposition material in the systems causing slight shifts in the thickness calibration. Once the substrates were removed from the thermal evaporator the grids where also removed. It is seen in figure 2.3, that the result was a pattern area of oxide thin films. For series A samples, the pattern oxide films are returned to the thermal evaporate without any TEM grids. A second layering of oxide material was deposited on top of the previous pattern. This second deposition always consisted of a different oxide than the first deposition. At this junction the substrate either has a pattern of $\mathrm{WO}_{3}$ with a full covering of $\mathrm{MoO}_{3}$ on top, or a pattern of $\mathrm{MoO}_{3}$ with a full covering of $\mathrm{WO}_{3}$. It is noted that $\mathrm{W}$ or Mo boats are used in order to control potential contamination. After both thermal evaporation steps are completed, a final CVD step is conducted. Figure 1.7, was a depiction of the CVD process. The setup is referred to as chalcogenization. A multi-zone quartz tube furnace is employed to convert the oxide material into sulfide or selenide systems. In the upstream zone, powdered or pellets of chalcogen atoms (sulfur or selenium) are placed in a glass vial that has the open end pointing downstream. A glass vial is used to assist carrier gases in directing the chalcogen atoms towards the oxide films in the middle zone. The 
chalcogenization step is done at atmospheric conditions. In order to prevent side reactions in the quartz tube, the system was purged with nitrogen at a volume flow rate of 500 sccm (standard cubic centimeter) for over 30 minutes. The above was found to be sufficient to remove ambient gases from the CVD system.

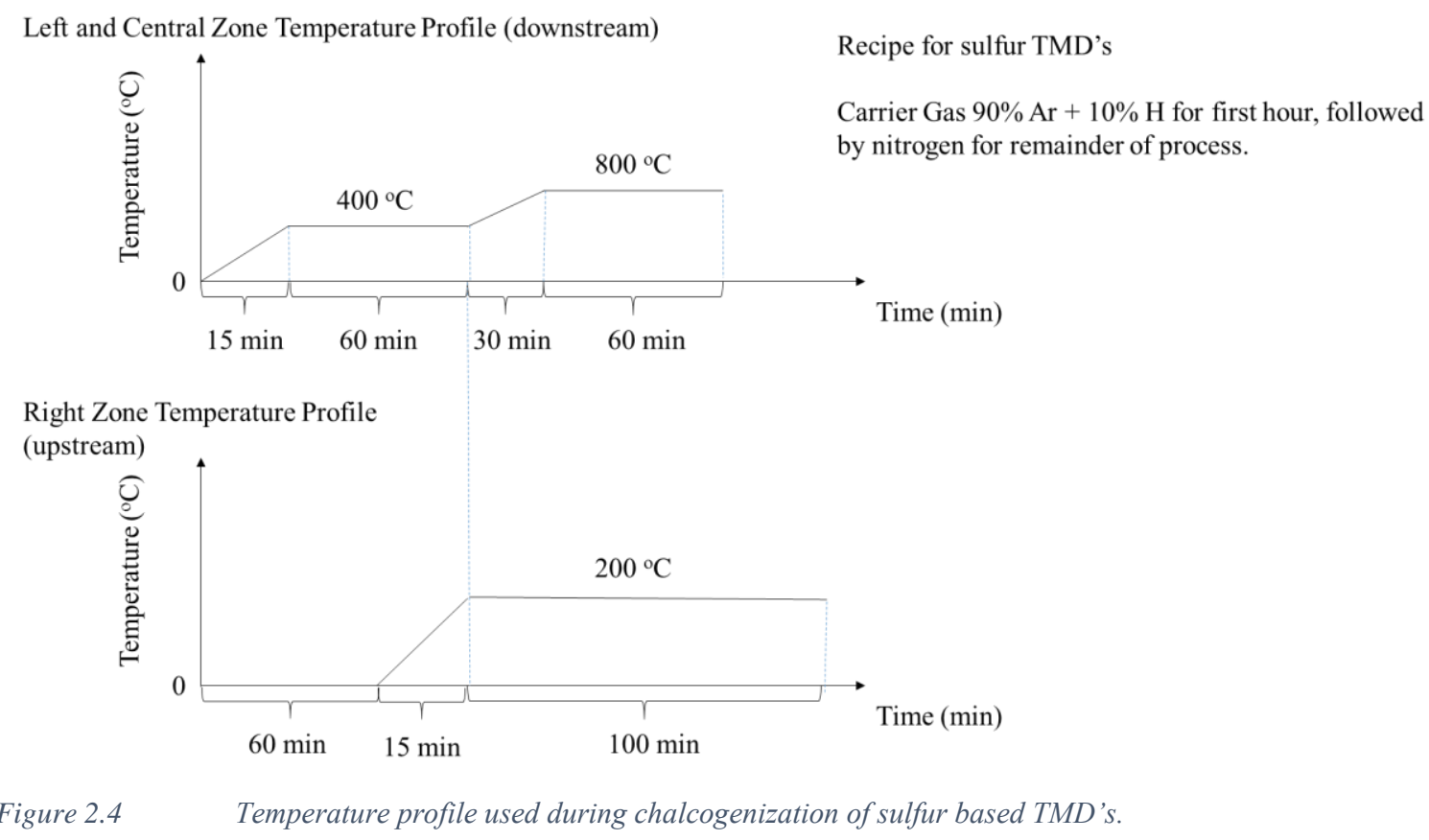

Temperature profile and ramping rates are shown in figure 2.4 and 2.5. There is a difference in the recipe for sulfur or selenium setups. Sulfur is covered first. During the first portion of the chalcogenization, the carrier gas used was argon balanced with $10 \%$ hydrogen. After the 30 minute nitrogen flush, the carrier gas is switched to the hydrogen mix. The argon/hydrogen gas was allowed to flow $(500 \mathrm{sccm})$ for about 5 minutes prior to heating the sample. Once the heating process begun, gas volume flow rate was 
decreased down to $200 \mathrm{sccm}$. The hydrogen mix served dual proposes. Preventing any ambient air from reaching the sample was its main job since the experiment was conducted at standard pressures. Its second job was for reducing the oxide films. Without the reduction step, $\mathrm{MoS}_{2}$ did not form. Temperature settings for left and central zones are the same. Once the furnace is activated the left and central zones heat from $25^{\circ} \mathrm{C}$ to 400 ${ }^{\circ} \mathrm{C}$ in 15 minutes. At $400{ }^{\circ} \mathrm{C}$ there is a dwell period of 60 minutes. As previously stated, the reduction of the trioxide species to $\mathrm{MoO}_{2-\mathrm{x}}$ and $\mathrm{WO}_{2-\mathrm{x}}$ subspecies $(\mathrm{x}<3)$ improves the probability of forming the TMD end product. Also as oxygen is removed the films, they become more stable and migrate less across the substrate. This decrease in surface diffusion helps retain the intricate patterns of material that had been deposited on the substrates through thermal evaporation. Next phase of the CVD process is ramping up to the desired temperature for TMD formation. A ramp for both left in middle zones, heats them from $400{ }^{\circ} \mathrm{C}$ to $800{ }^{\circ} \mathrm{C}$ in 30 minutes. Higher ramping rates to $800{ }^{\circ} \mathrm{C}$ caused issues with temperature overshoot and a subsequent heating of the upstream zone (which could lead to premature evaporation of the chalcogen atoms). Specifically $800{ }^{\circ} \mathrm{C}$ was chosen as the max temperature due to the melting point of $\mathrm{MoO}_{3}$ being at $795^{\circ} \mathrm{C}$. This had a redundancy aspect in that it forced the removal of any residual trioxide species. The furnace dwelled at $800{ }^{\circ} \mathrm{C}$ for 60 minutes (for conversion from $\mathrm{MoO}_{2-\mathrm{x}}$ and $\mathrm{WO}_{2-\mathrm{x}}$ subspecies to $\mathrm{MoS}_{2}$ and $\mathrm{WS}_{2}$ ). The right zone has a different heating process than the downstream zones. This difference is due to the large disparity in melting point of the oxide films compared to the chalcogen atom melting. Starting point for the upstream zone (right zone) begins at the same time as the left and central zones are stated. The zone actually sits at $25{ }^{\circ} \mathrm{C}$ for 60 minutes. This dwell allows for the oxide species to be 
reduced without being exposed to sulfur and prematurely starting the conversion process. After the dwell at room temperature, the carrier gas is switched from the hydrogen mix to pure nitrogen. At the same time that the carrier gases are switched, the right zone is heated from $25{ }^{\circ} \mathrm{C}$ to $200{ }^{\circ} \mathrm{C}$ in 15 minutes. Melting point of sulfur at atmospheric conditions is $115^{\circ} \mathrm{C}$. As such the sulfur beings to evaporate towards the end of the dwell at $400{ }^{\circ} \mathrm{C}$ for the central zone. Which ensures that the oxide films are being exposed to sulfur once they reach elevated temperatures. The right zone is held at $200{ }^{\circ} \mathrm{C}$ for 100 minutes, allowing the complete emersion of the samples in sulfur during the whole time at elevated temperatures. In addition as the central zone cools, the right zones also beings to cool before the central zone hits temperatures that would allow for the redeposition of pure sulfur. The volume flow is also increased to $500 \mathrm{sccm}$ to assist in removal of any remaining sulfur. Cooling of the samples was done by flowing air over the quartz tube.

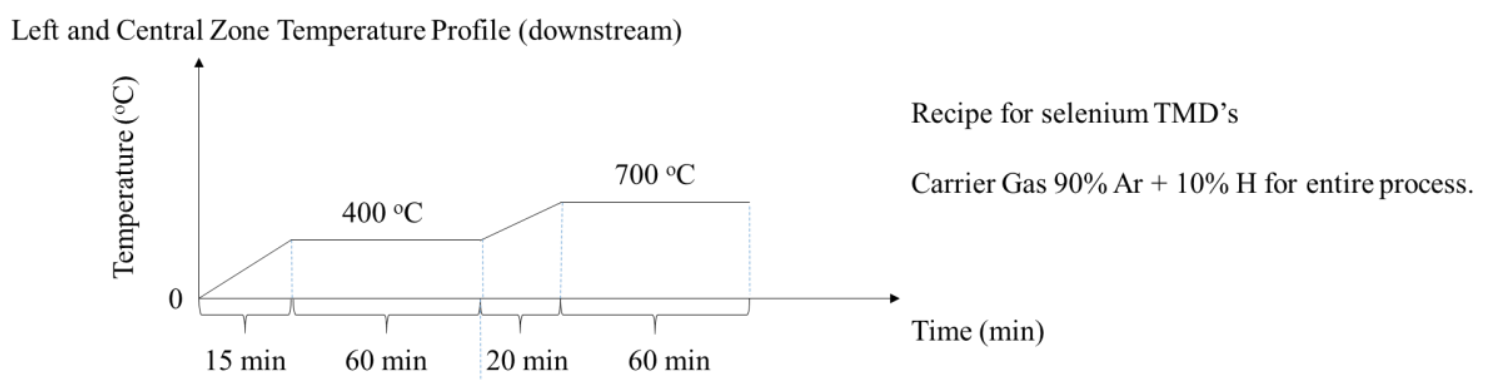

Right Zone Temperature Profile (upstream)

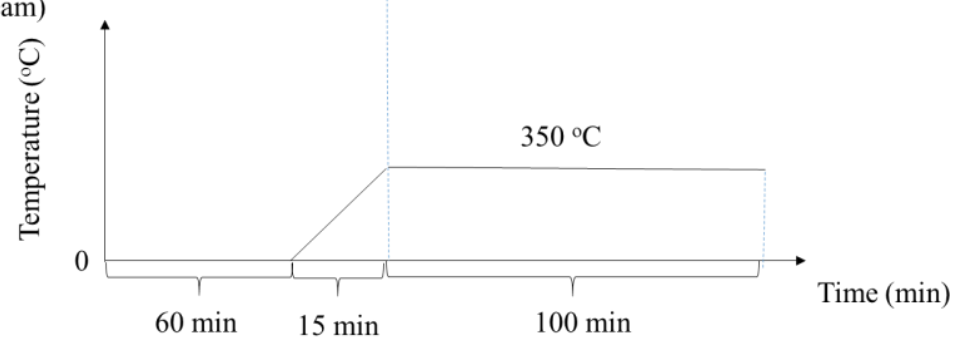

Figure 2.5 Temperature profile used during chalcogenization of selenium based TMD's 
For chalcogenization of selenium containing TMD's the CVD setup is similar to that of sulfur samples. The left and central zones are heated from $25^{\circ} \mathrm{C}$ to $400{ }^{\circ} \mathrm{C}$ in 15 minutes and stay at $400{ }^{\circ} \mathrm{C}$ for 60 minutes. The above step is the same as that of sulfur products due to the need to reduce the oxide species. After the reduction step the central and left zones are heated from $400{ }^{\circ} \mathrm{C}$ to $700{ }^{\circ} \mathrm{C}$ in 20 minutes. Unlike sulfur TMD's, the temperature at which selenium TMD's form is much lower. Using $800{ }^{\circ} \mathrm{C}$ for selenium TMD formation was not possible and all film material was lost. Holding the central and left zones at $700{ }^{\circ} \mathrm{C}$ for 60 minutes allowed for the conversion from $\mathrm{MoO}_{2-\mathrm{x}}$ and $\mathrm{WO}_{2-\mathrm{x}}$ subspecies to $\mathrm{MoSe}_{2}$ and $\mathrm{WSe}_{2}$. Due to the need for lower formation temperatures, the built in redundancy to remove residual trioxide species is not present. However, no issues arise with formation of other products outside of the desired $\mathrm{MoSe}_{2}$ or $\mathrm{WSe}_{2}$. The right zone (which contains the selenium pellets) has a much higher maximum temperature than that of the sulfur recipe. Which is in contrast to the central zone which has a lower maximum temperature than the sulfur recipe. Even though tellurium containing TMD's are not the focus of the current work, preliminary results from testing this recipe on their formation showed a similar trend. The format temperature of the central zone decreased by another $100{ }^{\circ} \mathrm{C}$ (down to $600{ }^{\circ} \mathrm{C}$ ) and the right zone maximum temperature increased to about $450{ }^{\circ} \mathrm{C}$ for preliminary recipe test of tellurium. For selenium TMD's the right zone has the same setup as that of the sulfur recipe. The maximum temperature is $350{ }^{\circ} \mathrm{C}$ and dwell at that temperature for 90 minutes. Decreased dwell time is needed to prevent deposition of pure selenium in the central zone due to selenium having a higher melting point. Therefore the selenium must be forced to cool down at a faster rate than sulfur. Once the carrier gas is switched after the initial purge process using nitrogen, it is never 
again switched back. Argon/hydrogen mix is used throughout the chalcogenization process due to no adverse effects being seen in the selenium samples. The reason for developing two different processes (Series A and Series B) was the end result of series A having more alloying between the tungsten layer and the molybdenum layer of the TMD heterostructure system. However, this effect will be addressed in detail in later sections.

Figure 2.3 also shows Series B. Branching point between Series A and Series B happens after the first patterned oxide deposition. In Series B, instead of placing the patterned film back into the thermal evaporator, it undergoes the CVD chalcogenization step. CVD temperature profile for sulfur products are the same as that of Series A. The same goes for the CVD recipes of selenium compounds. After the first chalcogenization of Series B, there is now a patterned TMD film on the substrate. The newly formed TMD is much more stable than its oxide counterpart and has much a higher melting point. The sample is now placed back into the thermal evaporator without any grid. A full coverage deposition of either $\mathrm{MoO}_{3}$ or $\mathrm{WO}_{3}$ is deposited on top of the already formed patterned TMD $\left(\mathrm{MoS}_{2}, \mathrm{WS}_{2}, \mathrm{MoSe}_{2}\right.$, or $\left.\mathrm{WSe}_{2}\right)$. The oxide/TMD system then undergoes one final chalcogenization step using either of the aforementioned CVD recipes.

One of the other key aspects of Series B, is that it can form heterostructures with different chalcogen atoms. In Series A, both materials in the heterostructure have the same chalcogen atom even though they have a different transition metal atoms. Such as $\mathrm{WS}_{2} / \mathrm{MoS}_{2}$ systems. This is due to Series A only having one chalcogenization. Series B can first form the highly stable $\mathrm{WS}_{2} \mathrm{TMD}$, followed by the growth of $\mathrm{WSe}_{2}$. The reason why this is important is because in TMD's, only monolayers have direct band gaps. Once multiply layers being to show up, the band gap shifts to indirect. There is however, a few 
special cases where this is not true. For systems that have differing chalcogen but common transition metal atoms, there are combinations that retain direct band gaps even for more than one layer [63]. The combination $\mathrm{WSe}_{2} / \mathrm{WS}_{2}$ has been predicted to be one such system. In addition, most monolayer films have low optical absorption due to its lack of substantial thickness. Increasing the number of layers, may improve this property by giving photons more time and space to interact with the films. This is a first step in making novel optoelectronic $2 \mathrm{D}$ based devices that may be able to be scaled up so that they have direct band gaps and are thick enough to compare to the penetration depth of light. It must be noted, that in order to grow the $\mathrm{WSe}_{2} / \mathrm{WS}_{2}$ heterostructures, $\mathrm{WS}_{2}$ had to be grown first. If $\mathrm{WSe}_{2}$ was grown first, the second chalcogenization to grow $\mathrm{WS}_{2}$ would cause the selenium in $\mathrm{WSe}_{2}$ to be exchanged with sulfur. The end result was multiply layers of $\mathrm{WS}_{2}$ with traces of $\mathrm{WSe}_{2}$ in random locations. It was also evident that the second chalcogenization (growth of $\mathrm{WSe}_{2}$ ) had to happen at temperatures less than the original recipe of $700{ }^{\circ} \mathrm{C}$ for selenium TMD's to keep replacement of sulfur with selenium to a minimum.

\subsection{Transfer of Transition Metal Dichalcogenide Heterostructures}

For certain characterization techniques (such as transmission electron microscopy) the heterostructures had to be transferred from the substrates used for growth to other substrates. A chemical etching process was employed. Due to the growth substrates being $\mathrm{SiO}_{2} / \mathrm{Si}$, potassium hydroxide $(\mathrm{KOH})$ was used. $\mathrm{KOH}$ was prepared by combining $20 \mathrm{~mL}$ of deionized water with $1.12 \mathrm{~g} \mathrm{KOH}$. 


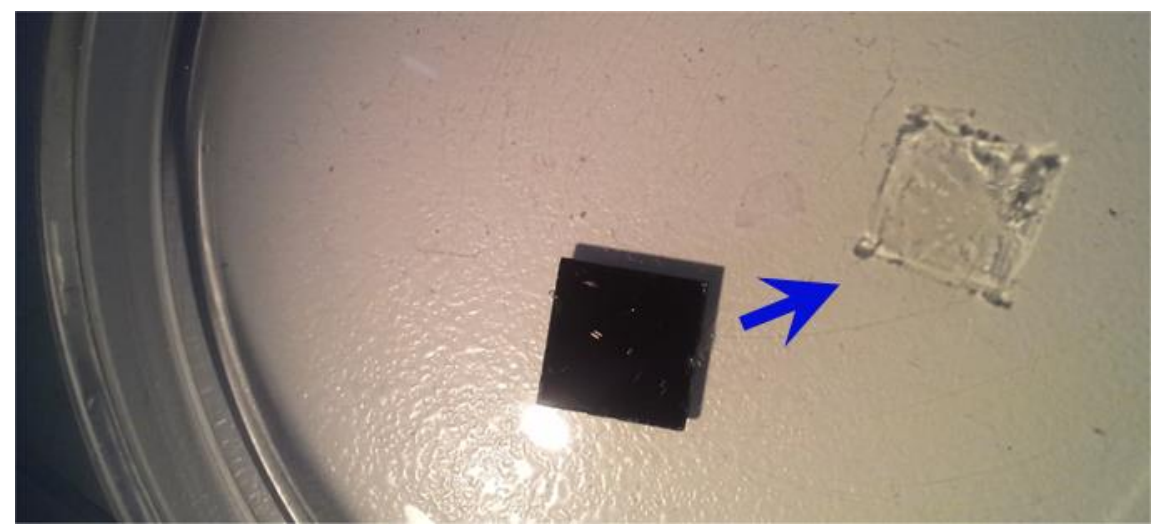

Figure 2.6

TMD film after it has been separated from $\mathrm{SiO}_{2} / \mathrm{Si}$ substrate

Before submersion of the samples into the $\mathrm{KOH}$, the heterostructures are spin coated. Spin coating a film of polymethyl methacrylate (PMMA) provides structural support for the heterostructures. The spin coat process used A5 PMMA. It was applied to the samples at $3000 \mathrm{rpm}$ for 60 seconds. In order for the PMMA to cure, it was dried overnight. After curing was completed, acetone was used to remove PMMA from the edges of the substrate. Without the above step, the PMMA acts a protective barrier preventing the $\mathrm{KOH}$ from etching the substrate. The samples were then submerged in the potassium hydroxide. $\mathrm{KOH}$ only etches the substrate. Eventually the substrate separates from the TMD heterostructures as shown by the floating film in figure 2.6. It was necessary to agitate the film slightly on its edges to facilitate separation. To remove any residual $\mathrm{KOH}$ from the heterostructure, the films are transferred to fresh deionized water. Heterostructure TMD's could now be applied to any substrate that does not degrade with water exposure. TMD samples are also allowed to dry after the transfer process. 


\subsection{Synthesis of Phosphorene}

The starting point for phosphorene synthesis is at bulk black phosphorus. Black phosphorus is currently being sold for over $\$ 400$ for $200 \mathrm{mg}$. Such a high price for small quantities of material has caused researchers to try to find ways to grow bulk black phosphorus. The current work uses a short transport growth method [80].

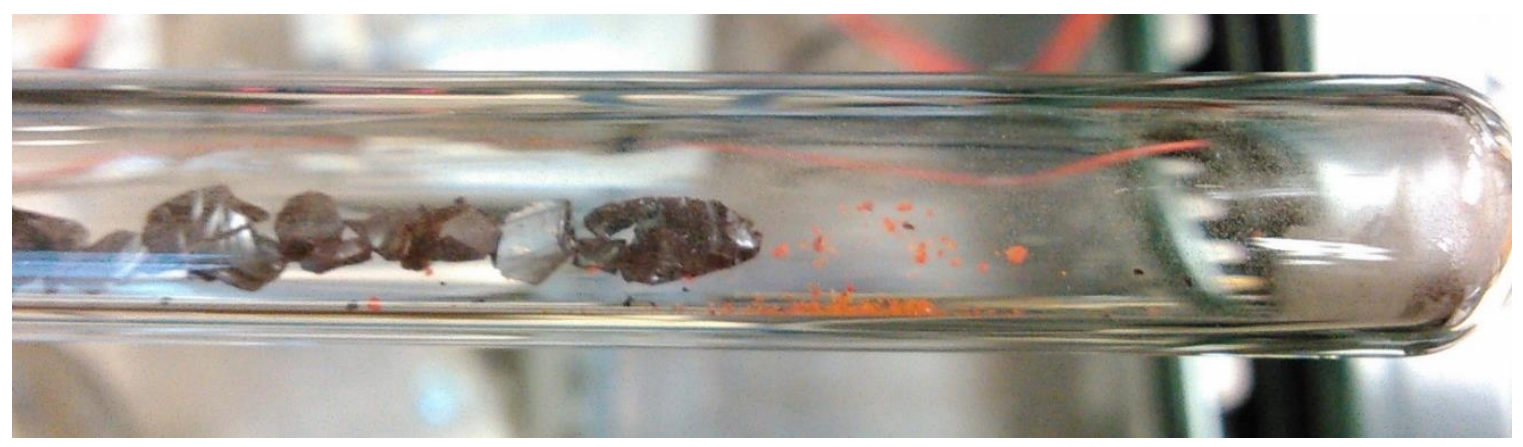

Figure 2.7 Precursor material for bulk black phosphorus synthesis inside glass tube during evacuation process

Precursor materials included bulk red phosphorus, $\mathrm{SnI}_{4}$, and tin. A Pyrex glass test tube was filled with $24 \mathrm{mg}$ tin, $12 \mathrm{mg} \mathrm{SnI}_{4}$, and $500 \mathrm{mg}$ red phosphorus in that order. Figure 2.7 shows a sample after precursors are added to the glass test tube. The open end of the test tube was then connected to a custom built vacuum system shown in figure 2.8 . Using a combination mechanical pump and oil diffusion pump, the test tube was evacuated down to 10-6 Torr. After evacuating the glass tube was sealed using an oxyacetylene torch (figure 2.9). To assist the sealing process the glass test tubes were customized by Custom Glassblowing of Louisville, Inc. to have a restriction. The restriction allowed the glass tube to be sealed faster. 


\section{Cold cathode}

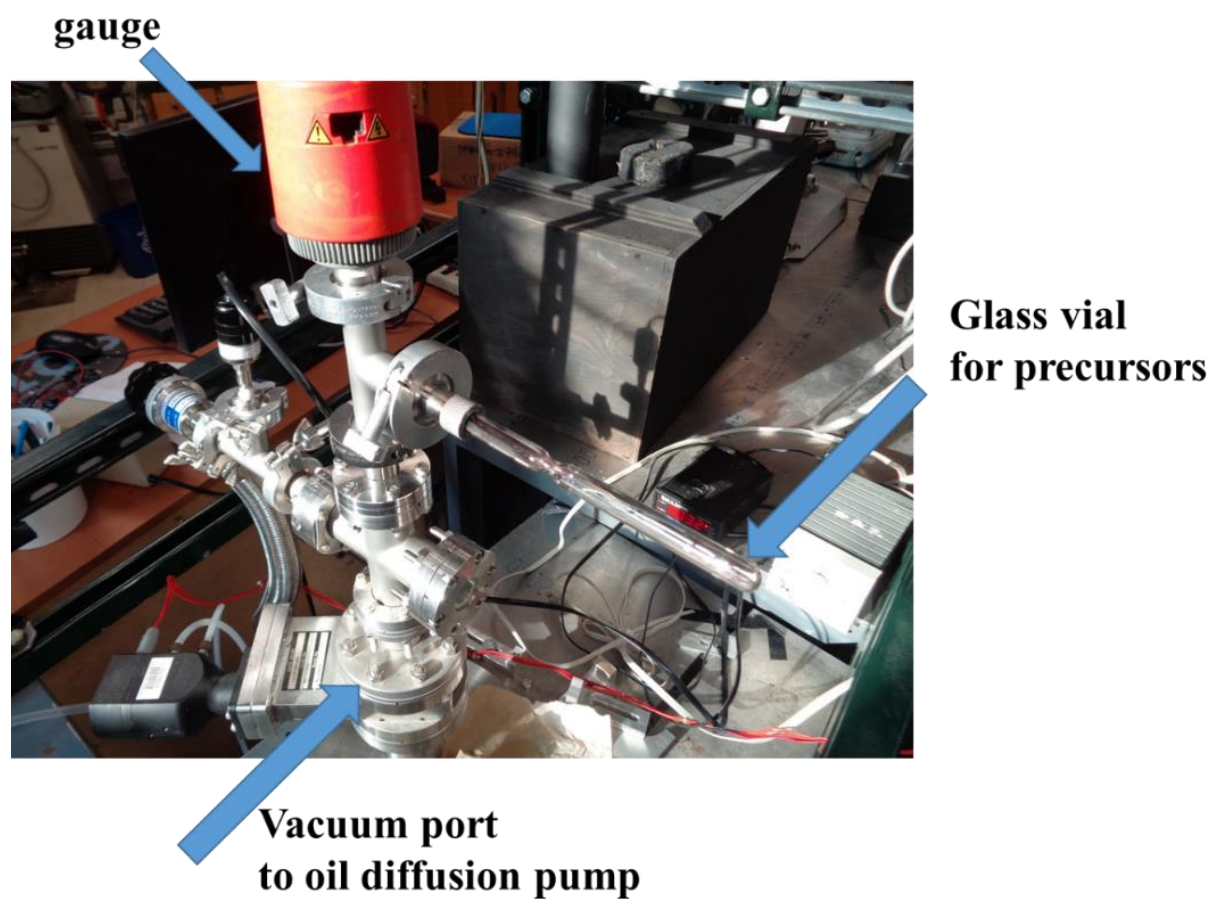

Figure $2.9 \quad$ Custom vacuum system for evacuating glass tubes
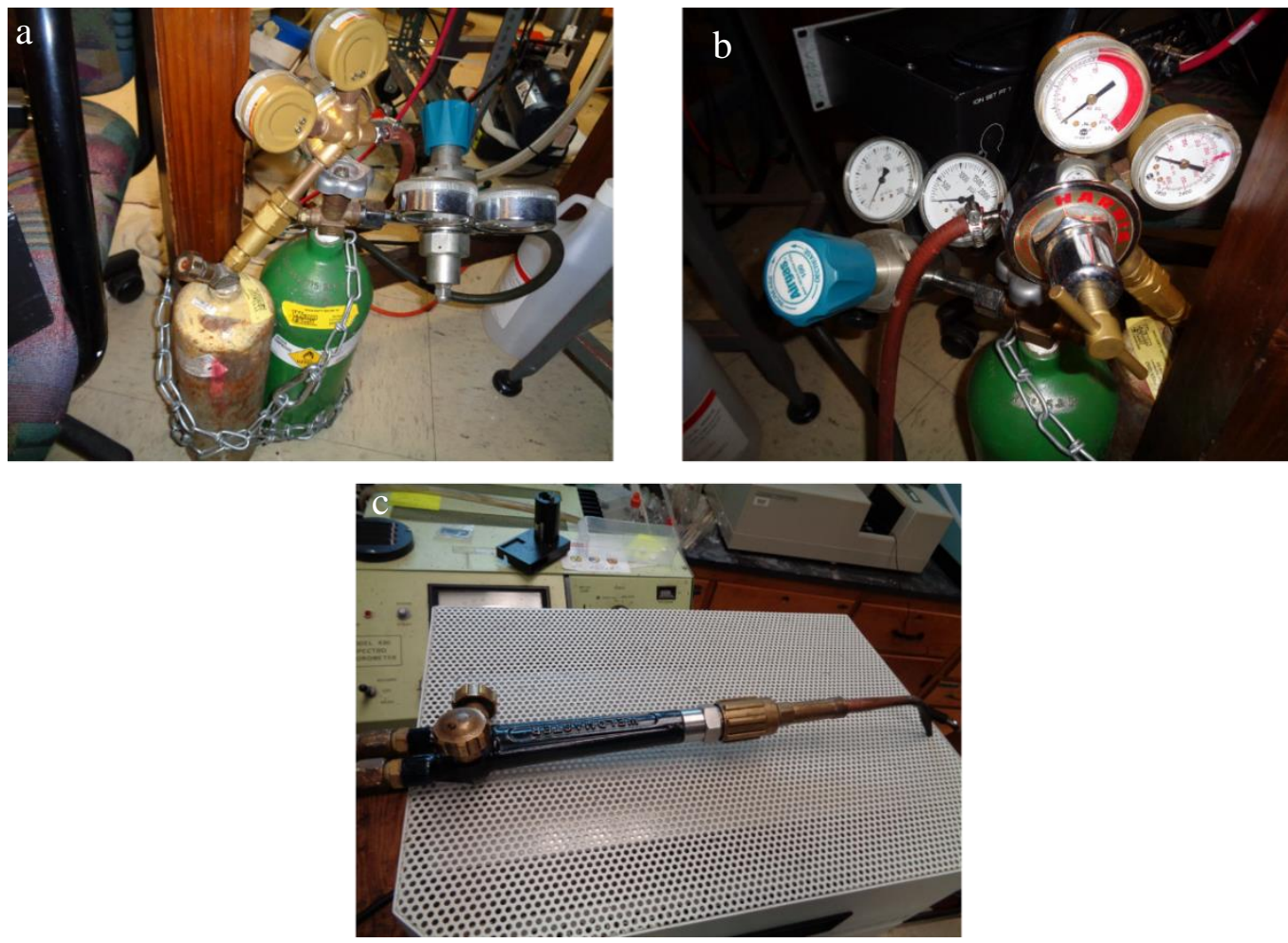

Figure 2.8

a.) and b.) Oxygen and acetylene tanks used for glass sealing. c.) Oxy-acetylene torch 


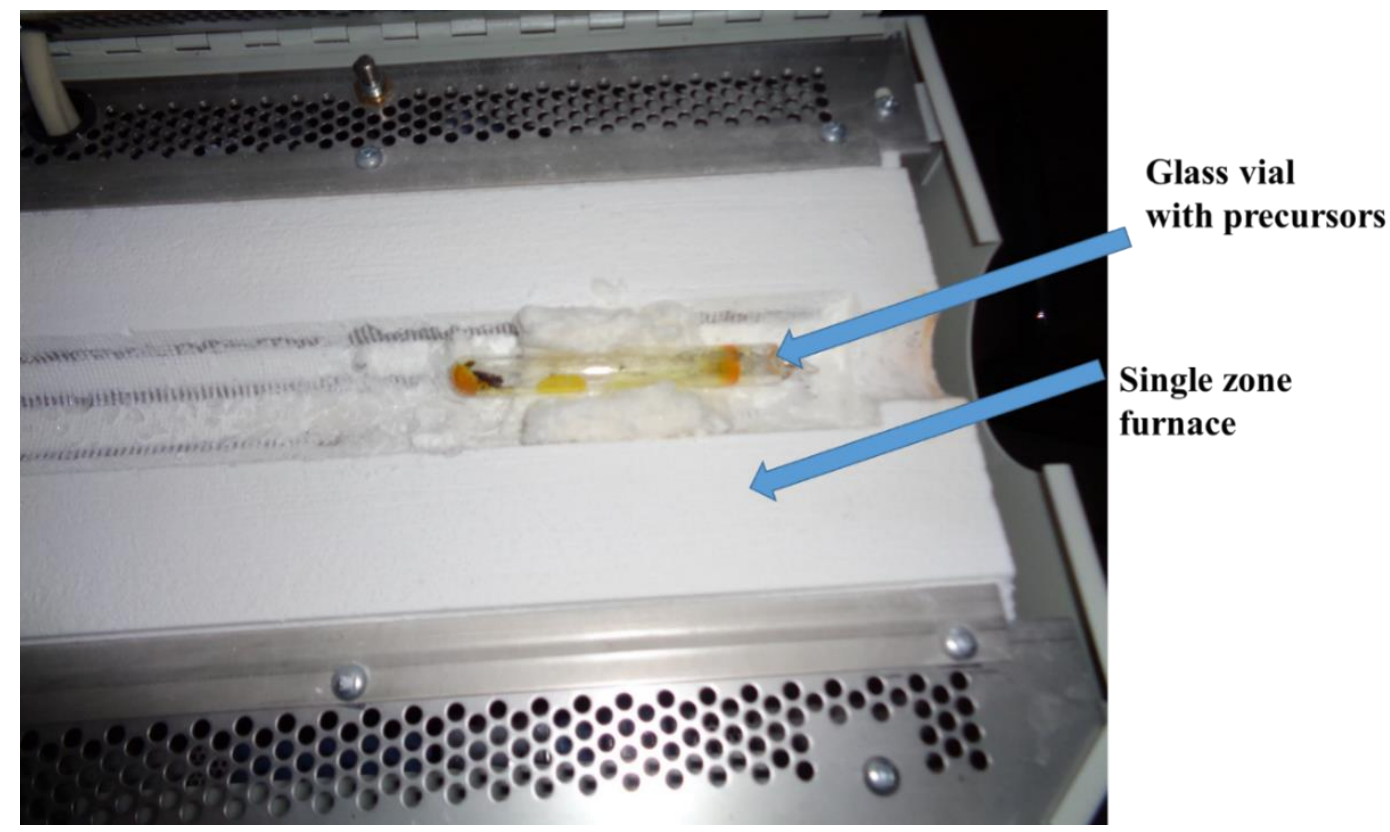

Growth of bulk black phosphorus was done in a single zone quartz tube furnace shown in figure 2.10. Temperature profile of the furnace is shown in figure 2.11. Initially the furnace is heated from room temperature to $610{ }^{\circ} \mathrm{C}$ over a period of 3 hours and 10 minutes. The sample was held at $610{ }^{\circ} \mathrm{C}$ for 20 minutes before being rapidly cooled to $560{ }^{\circ} \mathrm{C}$ within 5 minutes. Once $560{ }^{\circ} \mathrm{C}$ was reached the furnace took 7 hours and 34 minutes to cool down to $510^{\circ} \mathrm{C}$. There was a dwell at that temperature for one hour, followed a 5 hour and 11 minute cool down to room temperature. For the closed glass vial the end of it that contained the precursor material was placed in the hotter part of the furnace. This caused a temperature gradient of about $50{ }^{\circ} \mathrm{C}$ from the hotter end of the sealed Pyrex ampoule to the other end. Due to the use of Pyrex at $610^{\circ} \mathrm{C}$ the ampoule began to slowly expand. Taking the ampoule to a critical point happened after 20 minutes. Maintaining the ampoule above $600{ }^{\circ} \mathrm{C}$ at this point caused the ampoule to rupture. However, decreasing the temperature rapidly at the 20 minute mark, allowed the 
ampoule to retain its integrity. After the ampoule reached room temperature, large crystals of black phosphorus had formed within the ampoule. Figure 2.12 shows the ampoule after growth, however red phosphorus on the glass vials inner diameter hinders the view of black phosphorus. In order to prevent degradation of the black phosphorus, the crystals were stored in an argon glove box.

Recipe for bulk black phosphorus

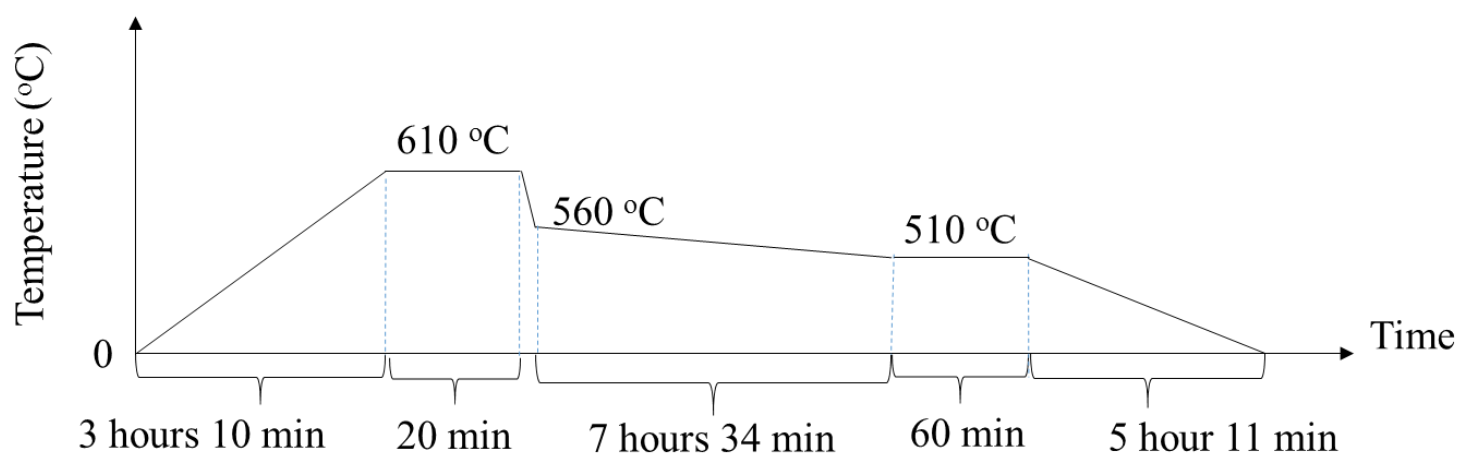

Figure 2.11 Temperature profile of furnace used in bulk black phosphorus synthesis 


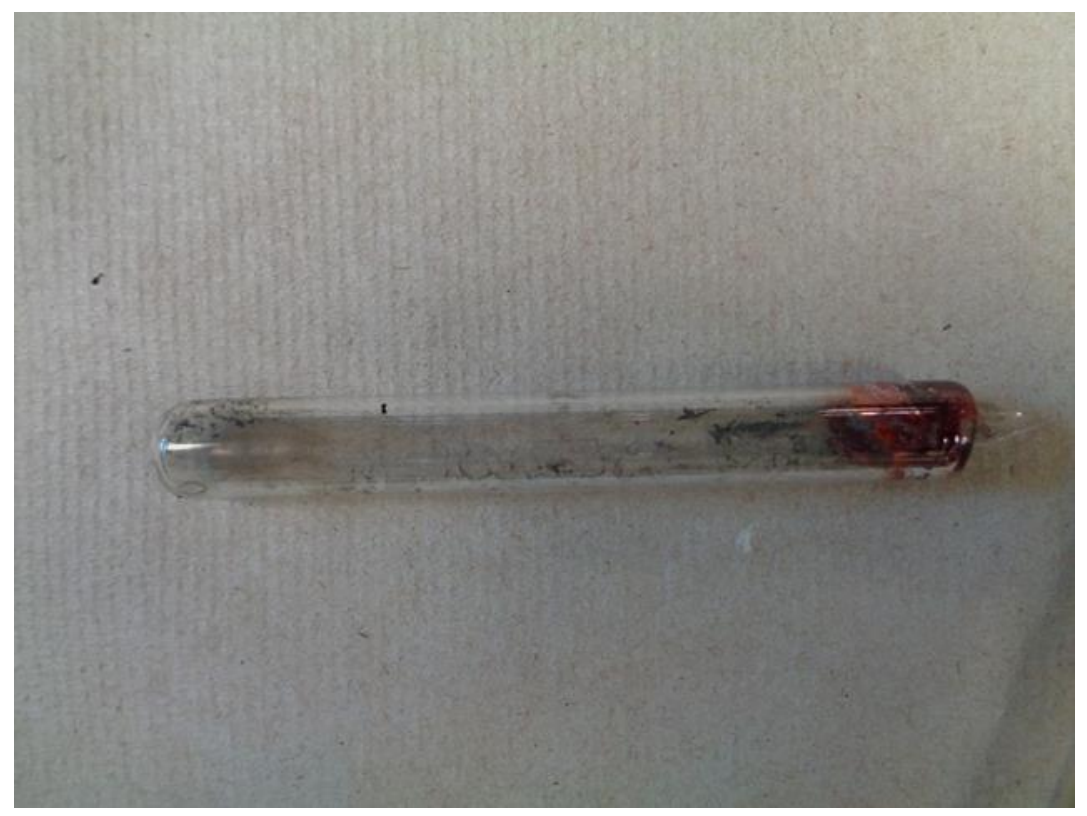

Figure 2.12 Glass tube after growth of bulk black phosphorus

For the liquid mechanical exfoliation (LME) process, $50 \mathrm{mg}$ of bulk black phosphorus was placed in a glass vial within the argon glove box. The black phosphorus was suspended in dimethylformalhide (DMF). In order to separate the phosphorene layers, the mixture was sonicated for 1 hour and 30 minutes. Previous research showed that monolayer phosphorene was formed by sonication for at least 15 hours [147]. The current work found that sonication less than one hour did not produce monolayers. Samples sonicated for over 1 hour contained 1-5 layers. Sonication times less than 1 hour formed 5 to 15 layers. After sonication, the samples underwent centrifugation. Constant temperature of $25^{\circ} \mathrm{C}$ was maintained by using a chilled centrifuge in order to decrease side reactions. The settings for the centrifuge were $10,000 \mathrm{rpm}$ for 30 minutes. Centrifugation caused higher layering number phosphorene (goes to bottom of vial) to separate from the lower layering number phosphorene. 


\subsection{Transition Metal Dichalcogenide Characterization}

Two main methods are used to analyze the heterostructure TMD's. They are raman spectroscopy and transmission electron microscopy. Raman was done on the original growth substrate of $\mathrm{SiO}_{2} / \mathrm{Si}$ due to the silicon vibrational mode $\left(520 \mathrm{~cm}^{-1}\right)$ allowing for proper calibration of the spectrums. For TEM, the heterostructures are transferred to TEM grids that have lacey carbon support networks. The proposed structures of the TMD heterostructures are shown in the inset of figure 2.3. The ensuing characterization of Series A and Series B, shows that Series A has a high interlayer exchange of transition metal atoms (alloying).

a

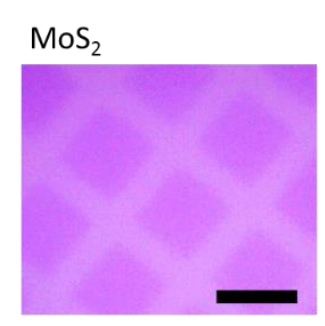

b
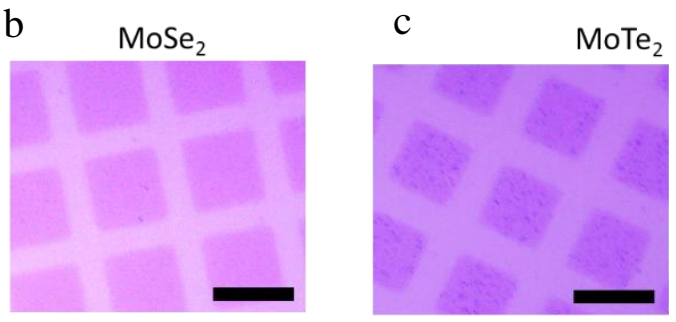

$\mathrm{d}$

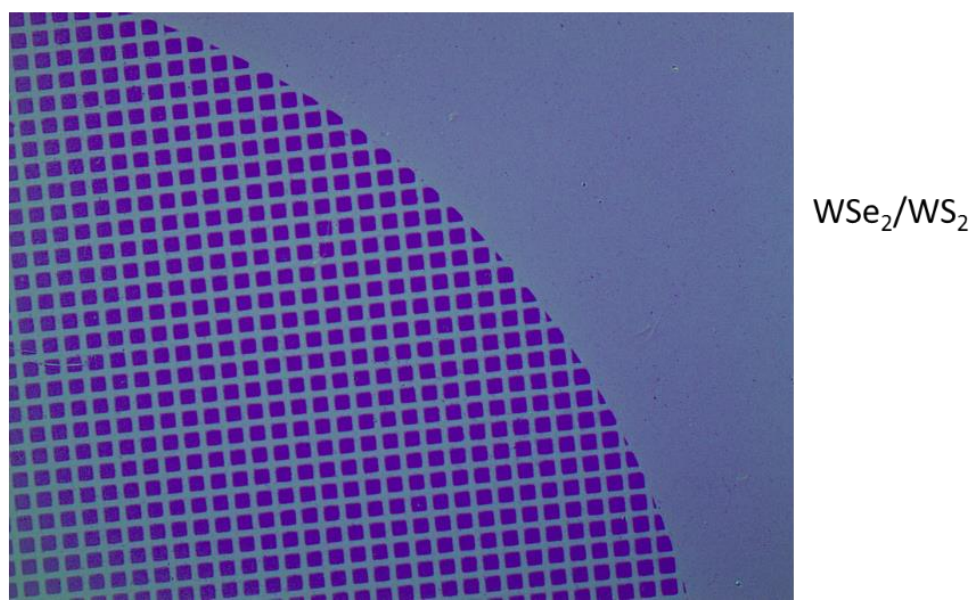

Figure $2.13 \quad$ Optical images of monolayer a.) MoS2, b.) MoSez, c.) MoTez and d.) bilayer $\mathrm{WSe}_{2} / \mathrm{WS}_{2}$

Figure $2.13 \mathrm{~d}$ shows an optical microscope image (the squares are about 10 by 10 $\mu \mathrm{m}$ ) of a series $\mathrm{B}$ bilayer sample of $\mathrm{WSe}_{2}$ on top of $\mathrm{WS}_{2}$. The figure also shows the 
molybdenum based monolayer TMD's after the first chalcogenization. It can be seen that the methods of the current work, can produce large area samples that have high visual uniformity.

\subsubsection{Raman Spectroscopy}
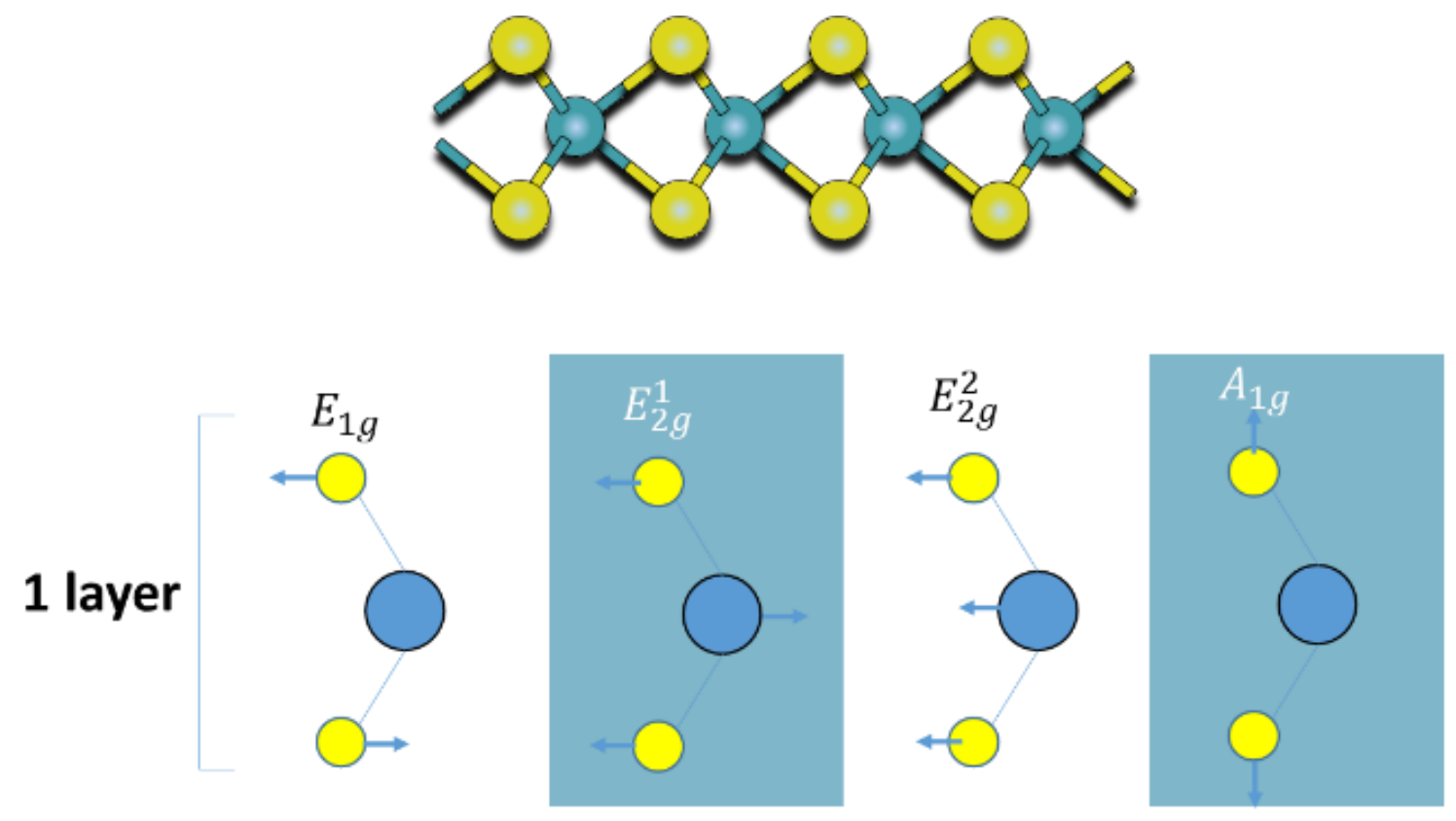

Figure 2.14 Vibrational modes of TMD's

The vibrational modes of $\mathrm{MoS}_{2}$ are shown in figure 2.14. The Second order peak $\mathrm{E}_{2 \mathrm{~g}}^{2}$ is too low (below $100 \mathrm{~cm}^{-1}$ ) [184]. Notch filter cuts off wavenumbers near the laser. The first order peak $\mathrm{E}_{1 \mathrm{~g}}$ is forbidden in backscattering geometry on a basal plane [184].

TMD's experience large changes in vibrational spectrums that correlate to changes in their physical properties. As the number of layers of $\mathrm{MX}_{2}(\mathrm{M}=\mathrm{Mo}, \mathrm{W} ; \mathrm{X}=\mathrm{S}$, 
Se) decrease their respective raman modes shift in characteristic manors. Determining the number of layers of $\mathrm{MoS}_{2}$ can be determined by analyzing the separation of the $\mathrm{A}_{1 \mathrm{~g}}$ and $\mathrm{E}^{1}{ }_{2 \mathrm{~g}}$ modes [20].

Characterization of nano-scale systems is important in relation to further understanding the capabilities of these materials. Raman spectroscopy is an effective method for analysis of vibrational properties of two dimensional structures. Depending on the characteristics of a particular set of elements, raman can be an quick method for chemical species identification, analysis, and polymorph determination [20, 185, 186]. Sir Chandrasekhara Venkata Raman and Sir Kariamanickam Srinivasa Krishnan discovered the raman process in 1928 [187].

The raman process is an excellent display of quantization. A material is irradiated with photons of energy $\Delta E=h v_{0}$. With h being Planck's constant and $v_{0}$ being the frequency of a photon. After light is scatted from the material, two different types of frequencies are collected. The original frequency $v_{0}$ is detected and defined as Rayleigh scattering [188]. Rayleigh scattering is the more intense of the different frequencies due to it being an elastic process with no change in energy. Another frequency, $v_{\mathrm{m}}$ is also collected. This secondary frequency is at a lower energy if a phonon (vibrational state transition) is created or at a higher energy if a phonon is absorbed. Stokes modes (phonon created) are defined as $v_{\text {phonon }}=v_{0}-v_{m}$, and $v_{\text {phonon }}=v_{0}+v_{m}$ is named AntiStokes modes (Phonon absorbed). Raman spectroscopy is a measure in the shift of frequency in relation to the excitation source, which is why it is also called raman shift. 


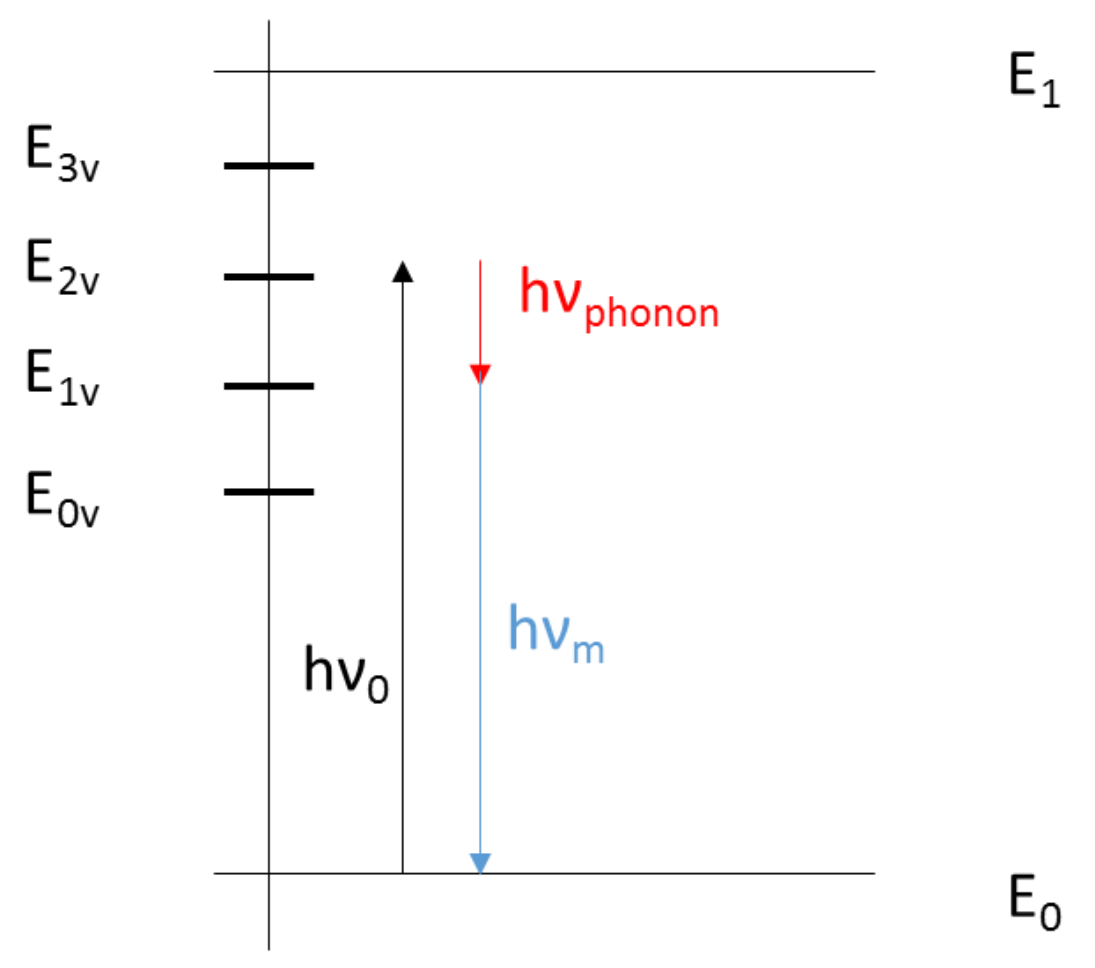

\section{Vibrational States}

Electronic States

Figure 2.15 Schematic of raman process

Figure 2.15 shows how a typical raman process happens in relation to the electronic state of system under study. The intermediate vibrational state during the transition is defined as being a virtual state in that it is not an electronic state or it is an unstable electronic state. Although it must be noted that the incident photon may have large enough energy to transition the molecule above or near higher intermediate electronic states before relaxation. The above effect is defined as resonance raman.

All possible raman vibration modes are not raman active. This arises due to the incident light beam inducing a dipole moment in the molecule. The resulting dipole 
moment has a direct dependence on the rate of change of the polarizability at the equilibrium position of the molecule. Zero rate of change results in a non-raman active mode. If the material is irradiated with a laser that can be approximated as having a sinusoidal monochromatic plane wave form, then the magnitude of the electric field it produces as a function of time can be defend as follows,

$$
E=E_{0} \cos 2 \pi v_{0} t
$$

$\mathrm{E}_{0}$ is the amplitude of the electromagnetic wave produced by the laser and $\mathrm{t}$ is time. The laser induces a dipole moment in the material and is approximated as being directly proportional to the electric field,

$$
p=\alpha E
$$

The variable $\alpha$ is defined as the atomic polarizability. Note that the above approximation is not valid if the electric field is too strong. With the material having a vibrational state of $v_{\mathrm{m}}$, the displacement of the molecule is q. For small vibrations $\alpha$ is a linear function of q. The atomic polarizability can be expanded as a Maclaurin series about the equilibrium position, in terms of displacement:

$$
\alpha=\alpha_{0}+\left(\frac{\partial \alpha}{\partial q}\right)_{0} q+\cdots
$$

Atomic polarizability at the equilibrium position is $\alpha_{0}$ and $(\partial \alpha / \partial q)_{0}$ is the displacement rate of change of the atomic polarizability at equilibrium. With the above equation, the dipole moment can be rewritten to a second order approximation as: 


$$
p=\left(\alpha_{0}+\left(\frac{\partial \alpha}{\partial q}\right)_{0} q\right) E
$$

Inserting the equation for $\mathrm{E}$ (eqn. 2.17) into the above equation:

$$
p=\left(\alpha_{0}+\left(\frac{\partial \alpha}{\partial q}\right)_{0} q\right) E_{0} \cos 2 \pi v_{0} t
$$

The form of $\mathrm{q}$ will also have a similar plane wave form as $\mathrm{E}$ and is as follows,

$$
q=q_{0} \cos 2 \pi v_{m} t
$$

With $\mathrm{q}_{0}$ being the vibrational amplitude. Combining the equation 2.22 for displacement with the dipole moment equation 2.21 we get equation:

$$
p=\alpha_{0} E_{0} \cos 2 \pi v_{0} t+\left(\frac{\partial \alpha}{\partial q}\right)_{0} q_{0} E_{0} \cos 2 \pi v_{0} t \cos 2 \pi v_{m} t
$$

Adding the two trigonometric identities:

$$
\begin{aligned}
& \cos A+B=\cos A \cos B-\sin A \sin B \\
& \cos A-B=\cos A \cos B+\sin A \sin B
\end{aligned}
$$

and combining them with the dipole moment equation 2.23 , the following is obtained.

$$
\begin{gathered}
p=\alpha_{0} E_{0} \cos 2 \pi v_{0} t \\
+\frac{1}{2}\left(\frac{\partial \alpha}{\partial q}\right)_{0} q_{0} E_{0} \cos 2 \pi\left(v_{0}+v_{m}\right) t+\frac{1}{2}\left(\frac{\partial \alpha}{\partial q}\right)_{0} q_{0} E_{0} \cos 2 \pi\left(v_{0}-v_{m}\right) t
\end{gathered}
$$


The first term is responsible for Rayleigh scattering. It is seen that the first derivative of the atomic polarizability with respect to displacement is directly proportional to the dipole moment in the last two terms. Anti-stokes Raman scattering is due to the second term, while the third term produces Stokes Raman scattering. As discussed earlier, for a Raman mode to be active the derivative of the atomic polarizability with respect to displacement must be non-zero.

The two main modes used to determine the number of layers in $\mathrm{MoS}_{2}$ are the inplane $\mathrm{E}_{2 \mathrm{~g}}^{1}$ mode and out of plane $\mathrm{A}_{1 \mathrm{~g}}$ mode [20]. For $\mathrm{MoS}_{2}$ the difference between the $\mathrm{A}_{1 \mathrm{~g}}$ and $\mathrm{E}_{2 \mathrm{~g}}^{1}$ modes are about $21.5 \mathrm{~cm}^{-1}$ and $19 \mathrm{~cm}^{-1}$ for bilayer and monolayer respectively [20]. $\mathrm{WS}_{2}$ displays the same effect with a difference of about $63 \mathrm{~cm}^{-1}$ and 60 $\mathrm{cm}^{-1}$ for bilayer and monolayer [1] for the same modes as $\mathrm{MoS}_{2}$. It has also been shown, that the absolute positions of the Raman modes for both $\mathrm{MoS}_{2}$ and $\mathrm{WS}_{2}$ are dependent on the laser excitation wavelength $[1,24]$. Settings used for raman study include 10 accumulations, 25 second exposure times, and 10\% laser power from $442 \mathrm{~nm}$ laser.

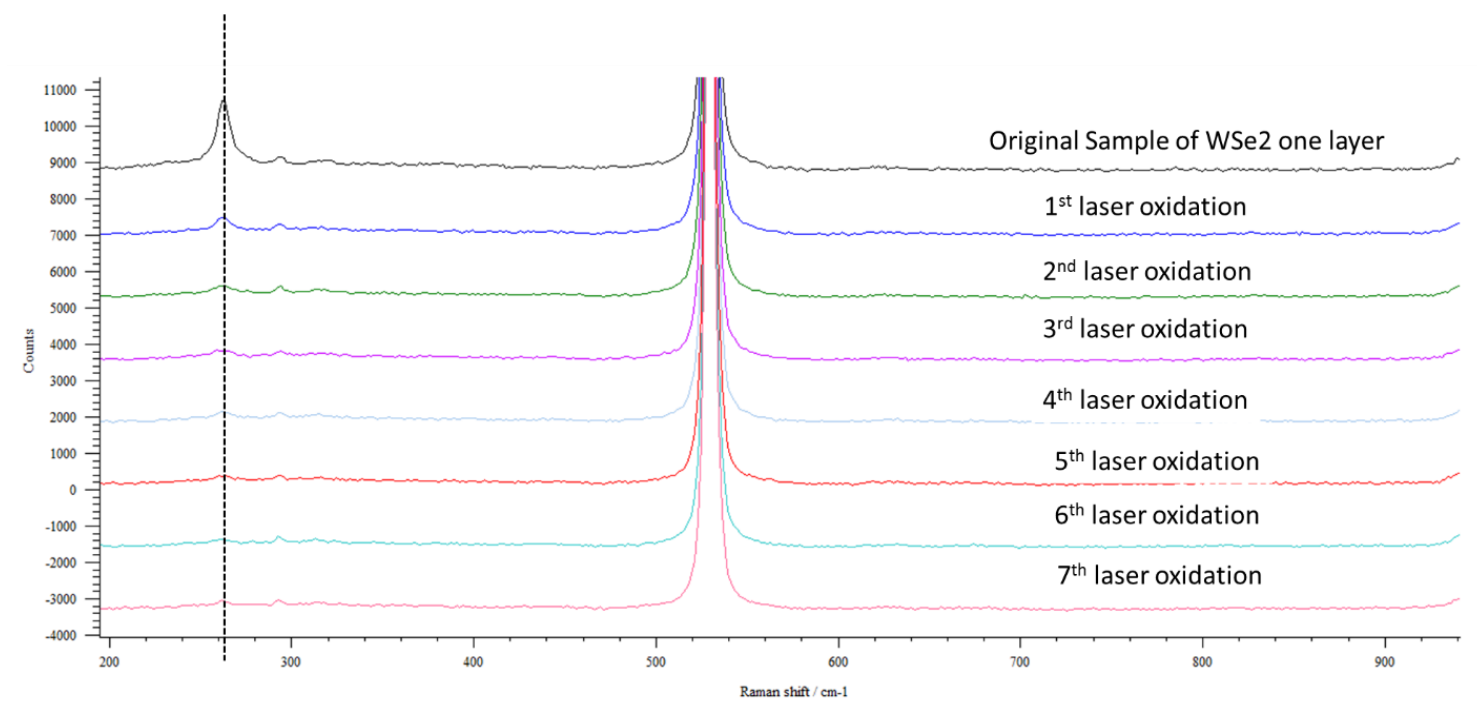

Figure 2.16 Raman spectrum of monolayer WSe2 exposed to laser several times 
Due to the use of lasers as the excitation source, care must be taken in order to prevent optical and thermal induced sample damage. For repeated laser exposure, it is suspected that material may become oxidized. The above effect is shown in figure 2.16 for WSe2. Each spectrum is done after the sample has been exposed. It is very evident that the intensity of the combination $\mathrm{E}^{1}{ }_{2 \mathrm{~g}} \mathrm{~A}_{1 \mathrm{~g}}$ peak is steadily decreasing as the sample is irradiated. Intensity of the peak decreases by almost $100 \%$ of its original intensity.
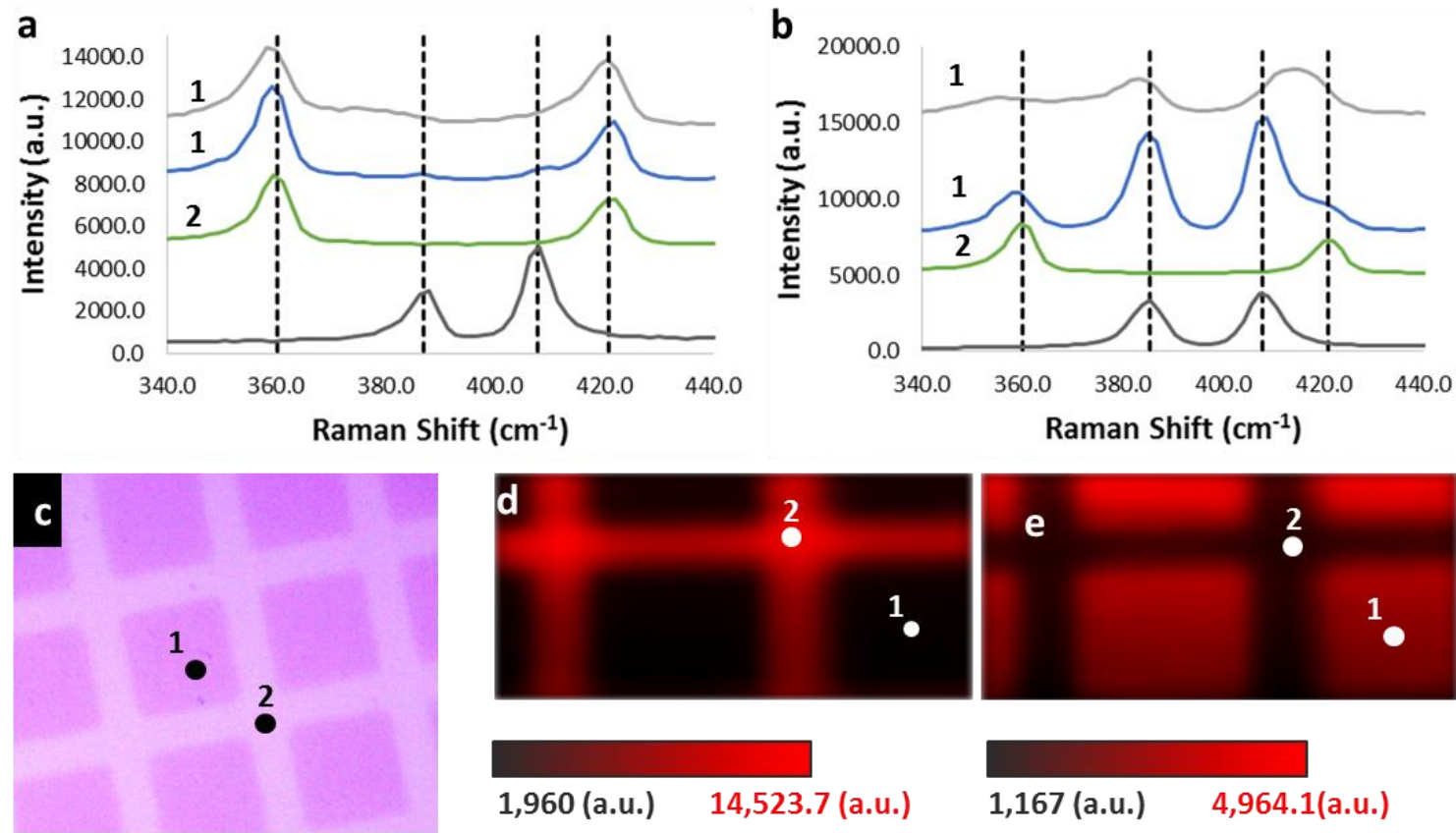

Figure 2.17 a.) Raman of bilayer $\mathrm{WS}_{2} / \mathrm{MoS}_{2}$ b.) Raman of monolayer $\mathrm{WS}_{2}$ on 4 layer $\mathrm{MoS}_{2}$ c.) Optical image showing locations used for raman measurements. d.) Raman mapping of $W_{2} E_{2 g}^{I}$ e.) Raman mapping of $M_{o S} E_{2 g}^{l}$

Figure 2.17a shows the Raman spectrum from monolayer $\mathrm{MoS}_{2}$, Series A bilayer $\mathrm{WS}_{2} / \mathrm{MoS}_{2}$ heterostructure and Series B bilayer $\mathrm{WS}_{2} / \mathrm{MoS}_{2}$ heterostructures with $\lambda=442$ $\mathrm{nm}$. Figure $2.17 \mathrm{~b}$ shows the same type of data except all $\mathrm{MoS}_{2}$ data is from 4 layers 
instead of monolayer. From figure $2.17 \mathrm{a}$, monolayer $\mathrm{MoS}_{2}$ raman spectrum shows two peaks. One peak is located at $387.0 \mathrm{~cm}^{-1}$ which is the $\mathrm{E}^{1}{ }_{2 \mathrm{~g}}$ for $\mathrm{MoS}_{2}$ and the other at 407.8 $\mathrm{cm}^{-1}$ is the $\mathrm{A}_{1 \mathrm{~g}}$ of $\mathrm{MoS}_{2}$. The difference between these peaks is $20.8 \mathrm{~cm}^{-1}$. Both the absolute positions and difference of the $\mathrm{A}_{1 \mathrm{~g}}-\mathrm{E}_{2 \mathrm{~g}}^{1}$ are in close agreement to previous experimental results for monolayer $\mathrm{MoS}_{2}$ [20]. For Series B at position 2 (outside the square) there are also two peaks, one at about $360 \mathrm{~cm}^{-1}$ and one at $420.8 \mathrm{~cm}^{-1}$. The peak at $360 \mathrm{~cm}^{-1}$ is actually the $2 \mathrm{LA}$ mode combined with the $\mathrm{E}^{1}{ }_{2 \mathrm{~g}}$ of $\mathrm{WS}_{2}$. Upon deconvolution of the peak, the $\mathrm{E}_{2 \mathrm{~g}}^{1}$ mode is identified at $360.3 \mathrm{~cm}^{-1}$ [1]. The peak at $420.8 \mathrm{~cm}^{-1}$ is the $\mathrm{A}_{1 \mathrm{~g}}$ mode of $\mathrm{WS}_{2}$ [1]. Difference of the $\mathrm{A}_{1 \mathrm{~g}}-\mathrm{E}_{2 \mathrm{~g}}^{1}$ is $60.5 \mathrm{~cm}^{-1}$ and is also in agreement with previous results for monolayer $\mathrm{WS}_{2}$. In addition, no peaks of $\mathrm{MoS}_{2}$ are found at position 2 in Series B. The above may be an indication of low alloying of Series $B$ in the in-plane direction and also high control of TMD species placement and control throughout the synthesis process. Series B at position 1 has 4 visible peaks which are the main modes of $\mathrm{WS}_{2}$ and $\mathrm{MoS}_{2}$. The $\mathrm{WS}_{2} \mathrm{E}_{2 \mathrm{~g}}^{1}$ at position 1 is shifted down to $359.3 \mathrm{~cm}^{-1}$ and the $\mathrm{A}_{1 \mathrm{~g}}$ stays about the same at $421.0 \mathrm{~cm}^{-1}$ with respect to $\mathrm{WS}_{2}$ signals at position 2 , for a difference of $61.7 \mathrm{~cm}^{-1}$. At position 1 Series $B$, the $\operatorname{MoS}_{2} \mathrm{E}_{2 \mathrm{~g}}{ }_{2 \mathrm{~g}}$ is shifted down to $386.1 \mathrm{~cm}^{-1}$ and the $\mathrm{A}_{1 \mathrm{~g}}$ is shifted up to $409.5 \mathrm{~cm}^{-1}$, which gives a difference of $23.4 \mathrm{~cm}^{-1}$. The same exact effects are shown in figure $2.17 \mathrm{~b}$ for Series $\mathrm{B}$, however the $\mathrm{MoS}_{2}$ peaks are more intense due to more layers of $\mathrm{MoS}_{2}$ and the shifts are more pronounced. The difference in $\mathrm{A}_{1 \mathrm{~g}}-\mathrm{E}^{1}{ }_{2 \mathrm{~g}}$ of $\mathrm{WS}_{2}$ has a slight increase to $62 \mathrm{~cm}^{-1}$ in position 2 of figure $2.17 \mathrm{~b}$ for Series $\mathrm{B}$, which is almost the same as the $61.7 \mathrm{~cm}^{-1}$ from figure $2.17 \mathrm{a} . \mathrm{MoS}_{2}$ has a similar increase in $\mathrm{A}_{1 \mathrm{~g}}-\mathrm{E}^{1}{ }_{2 \mathrm{~g}}$ of $23.6 \mathrm{~cm}^{-1}$ however the shift is actually smaller since the sample used in figure $2.17 \mathrm{~b}$ was for 4 layer and should already have a larger difference. 
Four layer pure $\mathrm{MoS}_{2}$ shown in figure $2.17 \mathrm{~b}$ has a difference of $22.68 \mathrm{~cm}^{-1}$. For Series B, when going from pure $\mathrm{WS}_{2}$ or $\mathrm{MoS}_{2}$ to the stacked structure of $\mathrm{WS}_{2} / \mathrm{MoS}_{2}$ the differences in $A_{1 g}-E^{1}{ }_{2 g}$ increases very slightly. Both the $E^{1}{ }_{2 g}$ and $A_{1 g}$ of both species are still visible in the stacked heterostructure area. Analysis of Series A in both figure 2.17a and $2.17 \mathrm{~b}$ (position 1) shows that there is no longer a distinction between the $\mathrm{A}_{1 \mathrm{~g}}$ of $\mathrm{MoS}_{2}$ and the $\mathrm{A}_{1 \mathrm{~g}}$ of $\mathrm{WS}_{2}$. In fact, in figure $2.17 \mathrm{~b}$ there is a peak between the $\mathrm{A}_{1 \mathrm{~g}}$ of pure $\mathrm{MoS}_{2}$ and the $\mathrm{A}_{1 \mathrm{~g}}$ of pure $\mathrm{WS}_{2}$. The above single peak has a larger full width at half maximum then the $\mathrm{A}_{1 \mathrm{~g}}$ of $\mathrm{MoS}_{2}$ (pure) and $\mathrm{WS}_{2}$ (pure) combined. It has been shown that for high alloying between $\mathrm{WS}_{2}$ and $\mathrm{MoS}_{2}$ the $\mathrm{A}_{1 \mathrm{~g}}$ mode of the combine system lies between the $\mathrm{A}_{1 \mathrm{~g}}$ of $\mathrm{MoS}_{2}$ and the $\mathrm{A}_{1 \mathrm{~g}}$ of $\mathrm{WS}_{2}$ [189]. The position of the combined peak shifts towards the species that is present in larger quantities [189]. The $\mathrm{MoS}_{2} \mathrm{~A}_{1 \mathrm{~g}}$ mode is altered (shifted) more when alloyed with $\mathrm{WS}_{2}$, as opposed to the $\mathrm{A}_{1 \mathrm{~g}}$ of $\mathrm{WS}_{2}$ [189]. The above may be an indicator of high alloying in Series A and low alloying in Series B.
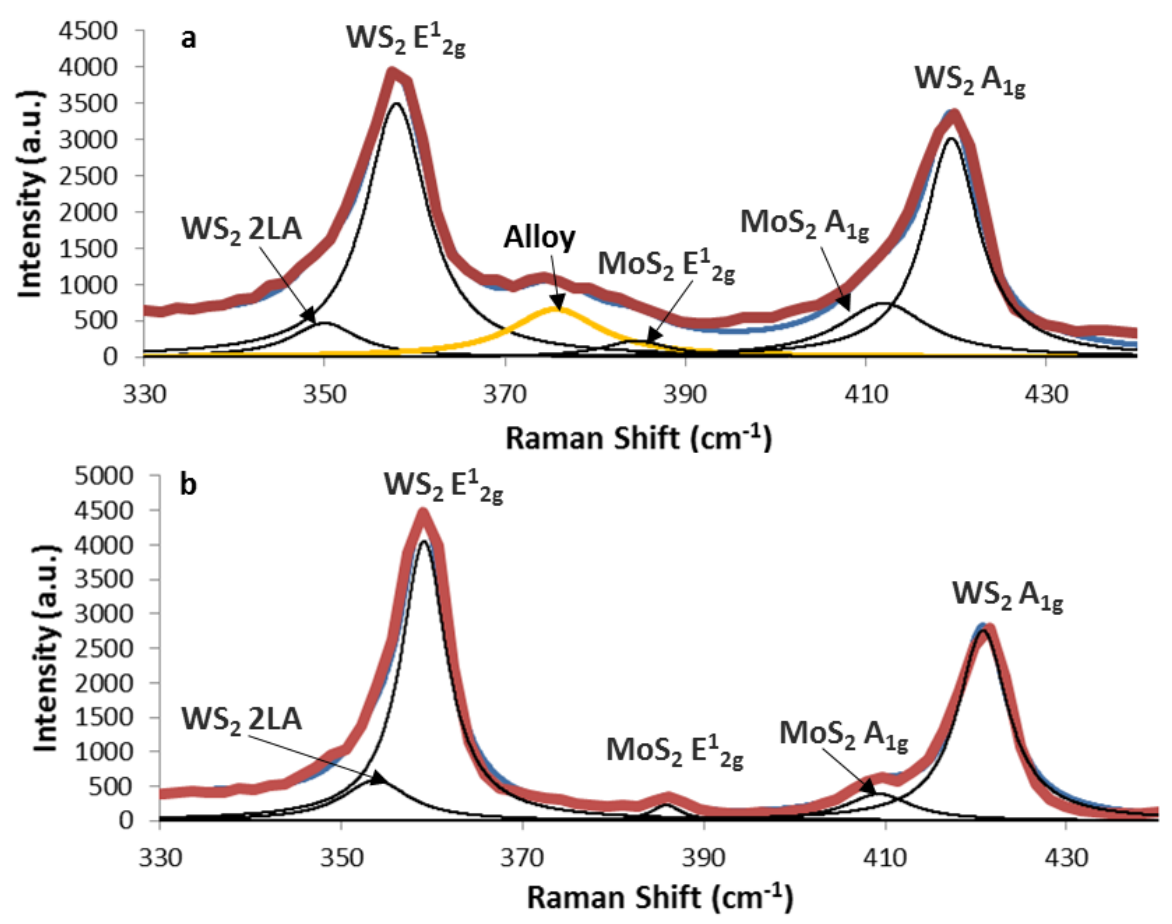

Figure 2.18 Deconvoluted raman of a.) bilayer $\mathrm{WS}_{2} / \mathrm{MoS} \mathrm{S}_{2}$ series $A$. b.) bilayer $\mathrm{WS}_{2} / \mathrm{MoS} \mathrm{S}_{2}$ series $B$ 
Series A and Series B (1 layer $\mathrm{WS}_{2}$ on top of 1 layer $\mathrm{MoS}_{2}$ ) Raman spectrum after deconvolution are shown in figure 2.18. In Series A, a peak arises that is not associated with $\mathrm{WS}_{2}$ or $\mathrm{MoS}_{2}$. This peak is not present in Series B (figure 2.18b). This peak is identified as an alloy disorder related peak, and is due to alloying between $\mathrm{WS}_{2}$ and $\mathrm{MoS}_{2}$ [189]. Figure $2.17 \mathrm{~d}$ and $2.17 \mathrm{e}$ are Raman intensity mappings of a Series A sample with 1 layer $\mathrm{WS}_{2}$ on top of 4 layers $\mathrm{MoS}_{2}$. Multiple layers of $\mathrm{MoS}_{2}$ were used in this sample due to the low intensity of the $\mathrm{MoS}_{2}$ raman peaks for monolayer $\mathrm{MoS}_{2}$ once it is combined into a Series A heterostructure. The maps were done for the $\mathrm{E}^{1}{ }_{2 \mathrm{~g}}$ modes of both $\mathrm{WS}_{2}$ (figure 2.17d) and $\mathrm{MoS}_{2}$ (figure 2.17e). The intensity of the $\mathrm{E}^{1}{ }_{2 \mathrm{~g}}$ for $\mathrm{MoS}_{2}$ drops drastically (figure 2.17e) as the map approaches region 2 (outside the square). This is due to the original deposition of patterned $\mathrm{MoO}_{3}$ in Series A, in which the resulting $\mathrm{MoS}_{2}$ retains its general pattern. It is apparent from figure 2.17e that although the drop in intensity is large from region 1 to region 2 , there is a gradual transition between the two regions. This transition area indicates that in Series A, there may also be in-plane alloying in addition to the aforementioned alloying in the stacked heterostructure region. The difference in alloying between Series A and Series B may be attributed to the process by which the respective samples are synthesized. Series B method has two chalcogenization steps as shown in figure 2.3. The first chalcogenization allows $\mathrm{MoO}_{3}$ to convert to $\mathrm{MoS}_{2}$. The $\mathrm{MoS}_{2}$ is more stable (higher melting point) then $\mathrm{MoO}_{3}$ at the temperatures in which chalcogenization process utilizes. The above may indicate that during the single chalcogenization process of Series $\mathrm{A}$, the $\mathrm{MoO}_{3}$ has more mobility and Mo atoms may interchange with $\mathrm{W}$ (from $\mathrm{WO}_{3}$ before it forms $\mathrm{WS}_{2}$ ). In Series B the $\mathrm{MoS}_{2}$ has less 
mobility during the final chalcogenization process. In turn, molybdenum's ability to exchange with tungsten atoms is drastically limited in Series B.
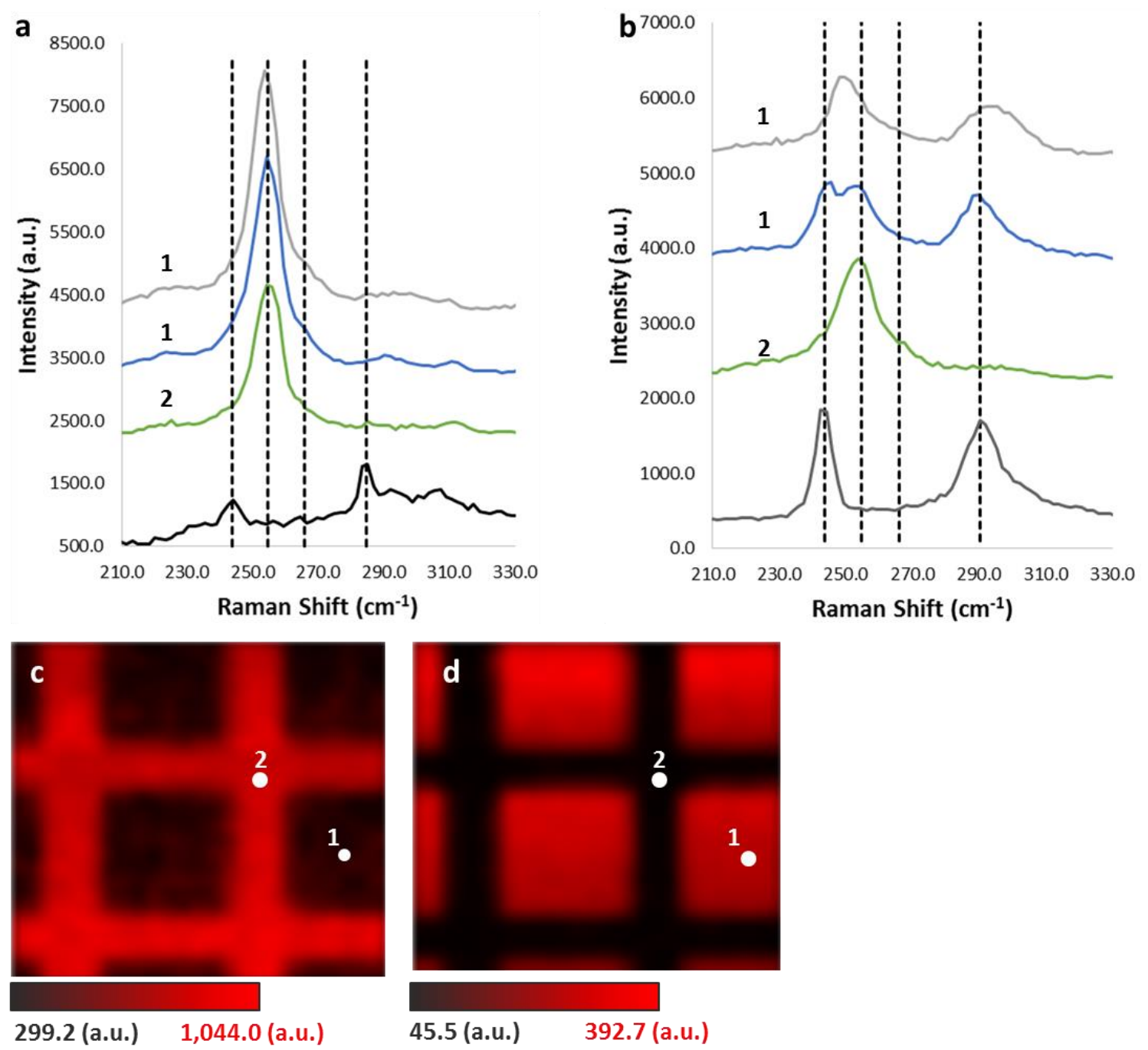

Figure $2.19 \quad$ a.) Raman of bilayer $\mathrm{WSe}_{2} / \mathrm{MoSe}_{2}$ b.) Raman of monolayer $\mathrm{WSe}_{2}$ on 4 layer $\mathrm{MoSe}_{2}$ c.) Raman mapping of $\mathrm{WS}_{2}$ degenerate $E_{2 g}^{\mathrm{I}}{ }_{2} A_{l g} d$.) Raman mapping of $\mathrm{MoSe}_{2} E_{2 g}{ }_{2 g}$

Series A and Series B with Se chalcogen atoms have been synthesized. Raman analysis of Series A and Series B WSe 2 on top of $\mathrm{MoSe}_{2}$ is shown in figure 2.19. The same affects that were seen for $\mathrm{WS}_{2} / \mathrm{MoS}_{2}$ samples are seen for $\mathrm{WSe}_{2} / \mathrm{MoSe}_{2}$ samples. $\mathrm{MoSe}_{2}$ has two characteristic modes used to determine number of layers the $\mathrm{A}_{1 \mathrm{~g}}$ and $\mathrm{E}_{2 \mathrm{~g}}^{1}$ 
vibrational modes [14]. The $\mathrm{A}_{1 \mathrm{~g}}$ mode is located around $240.5 \mathrm{~cm}^{-1}$ and the $\mathrm{E}_{2 \mathrm{~g}}^{1}$ mode is about $287.2 \mathrm{~cm}^{-1}$ for monolayer $\mathrm{MoSe}_{2}$ [14].This gives a difference of $46.7 \mathrm{~cm}^{-1}$. Figure 2.19a and figure $2.19 \mathrm{~b}$ shows that for pure $\mathrm{MoSe}_{2}$ both modes are present. It also shows that in region 2 no $\mathrm{MoSe}_{2}$ modes are found for Series B. The above suggest that no inplane alloying has occurred. For monolayer pure $\mathrm{MoSe}_{2}$ the difference of $\mathrm{E}^{1}{ }_{2 \mathrm{~g}}-\mathrm{A}_{1 \mathrm{~g}}$ was found to be $40.9 \mathrm{~cm}^{-1}$, which is less than previous experimental values. Four layer $\mathrm{MoSe}_{2}$ (Figure 2.19b) has a difference of $47.34 \mathrm{~cm}^{-1}$, which is larger than the corresponding monolayer value as expected. For pure $\mathrm{WSe}_{2}$ (region 2 for Series B) we have one peak at $252.8 \mathrm{~cm}^{-1}$ in figure $2.19 \mathrm{a}$ and one at $253.6 \mathrm{~cm}^{-1}$ in figure $2.19 \mathrm{~b}$. This may be attributed to the $\mathrm{E}^{1}{ }_{2 \mathrm{~g}}$ and $\mathrm{A}_{1 \mathrm{~g}}$ modes of $\mathrm{WSe}_{2}[14,15]$. However, research has shown that the $\mathrm{E}^{1}{ }_{2 \mathrm{~g}}$ and $\mathrm{A}_{1 \mathrm{~g}}$ modes for $\mathrm{WSe}_{2}$ are in close proximity to one another and are almost degenerate $[14,15]$. Due to the above degeneracy, deconvolution of the peaks to determine the location of the $\mathrm{E}_{2 \mathrm{~g}}^{1}$ and $\mathrm{A}_{1 \mathrm{~g}}$ may not be possible. In the current work the above mode is assigned as the $\mathrm{E}_{2 \mathrm{~g}}^{1} \mathrm{~A}_{1 \mathrm{~g}}$ combined mode of $\mathrm{WSe}_{2}$ for clarity purposes. Although the above Raman mode by itself cannot determine layer thickness, research has shown that for few layer $\mathrm{WSe}_{2}$ an additional peak forms between $5-11 \mathrm{~cm}^{-1}$ from the combinational mode[15]. This peak is located $11 \mathrm{~cm}^{-1}$ away from the combination mode for $\mathrm{WSe}_{2}$ only in monolayer films and decreases as more layers are added [15]. In figure 2.19a and $2.19 \mathrm{~b}$, an asymmetric shoulder can be seen on the combination mode towards higher Raman shift values. This shoulder is the additional peak that can be used to identify layering number in few layer WSe 2 . In figure $2.19 \mathrm{~b}$ for region 2 of Series B there is a difference of $12.2 \mathrm{~cm}^{-1}$ between the shoulder peak and the combination peak for $\mathrm{WSe}_{2}$. For figure 2.19a for region 2 of Series B the difference is $11.4 \mathrm{~cm}^{-1}$. Both values agree 
with previously reported values for monolayer $\mathrm{WSe}_{2}$. Figure $2.19 \mathrm{~b}$ shows that for Series $\mathrm{B}$ in region 1 we can distinctly see both $\mathrm{A}_{1 \mathrm{~g}}$ and $\mathrm{E}_{2 \mathrm{~g}}^{1}$ modes of $\mathrm{MoSe}_{2}$. Both $\mathrm{MoSe}_{2}$ are also present in figure 2.19a Series B in region 1, however there intensity is extremely low. The $\mathrm{E}^{1}{ }_{2 \mathrm{~g}} \mathrm{~A}_{1 \mathrm{~g}}$ combined mode of $\mathrm{WSe}_{2}$ is also present. In Series A conversely, the distinction can no longer be made between the $\mathrm{A}_{\mathrm{gg}}$ of $\mathrm{MoSe}_{2}$ and the combination mode of $\mathrm{WSe}_{2}$. Just as sulfur samples had similar findings, it may be evident that Series A selenium samples have high alloying. The Series B selenium samples may also have low alloying, just as its sulfur counterpart. The Raman intensity maps of the selenium Series A samples (figure $2.19 \mathrm{~d}$ and $2.19 \mathrm{e}$ ) appear almost exactly as the raman intensity maps for the sulfur Series A samples. Yet, one major difference can be seen in figure $2.19 \mathrm{e}$ for the $\mathrm{E}_{2 \mathrm{~g}}{ }_{\mathrm{g}}$ of $\mathrm{MoSe}_{2}$. There does not appear to be a transition area between regions 1 and 2. There is an abrupt drop in intensity of the $\mathrm{E}^{1}{ }_{2 \mathrm{~g}}$ for $\mathrm{MoSe}_{2}$ as the map leaves the square area (region 1). A distinct change in intensity shows that for Series A WSe $2 / \mathrm{MoSe}_{2}$, the in-plane alloying may be comparable to Series B WSe$/ \mathrm{MoSe}_{2}$. This was not the case for the sulfur samples. The above phenomenon may be due to the lower temperatures used during the chalcogenization process (max temperature of $700{ }^{\circ} \mathrm{C}$ instead of $800{ }^{\circ} \mathrm{C}$ ) of the selenium heterostructures. Even during the single chalcogenization process for Series A, the $\mathrm{MoO}_{3}$ has less mobility at $700{ }^{\circ} \mathrm{C}$ and hinders only in-plane alloying. However, alloying is still apparent in the stacked heterostructure section (region 1). This may be an indication that more energy is needed to provide in-plane mobility as opposed to mobility between the stacked structures. 


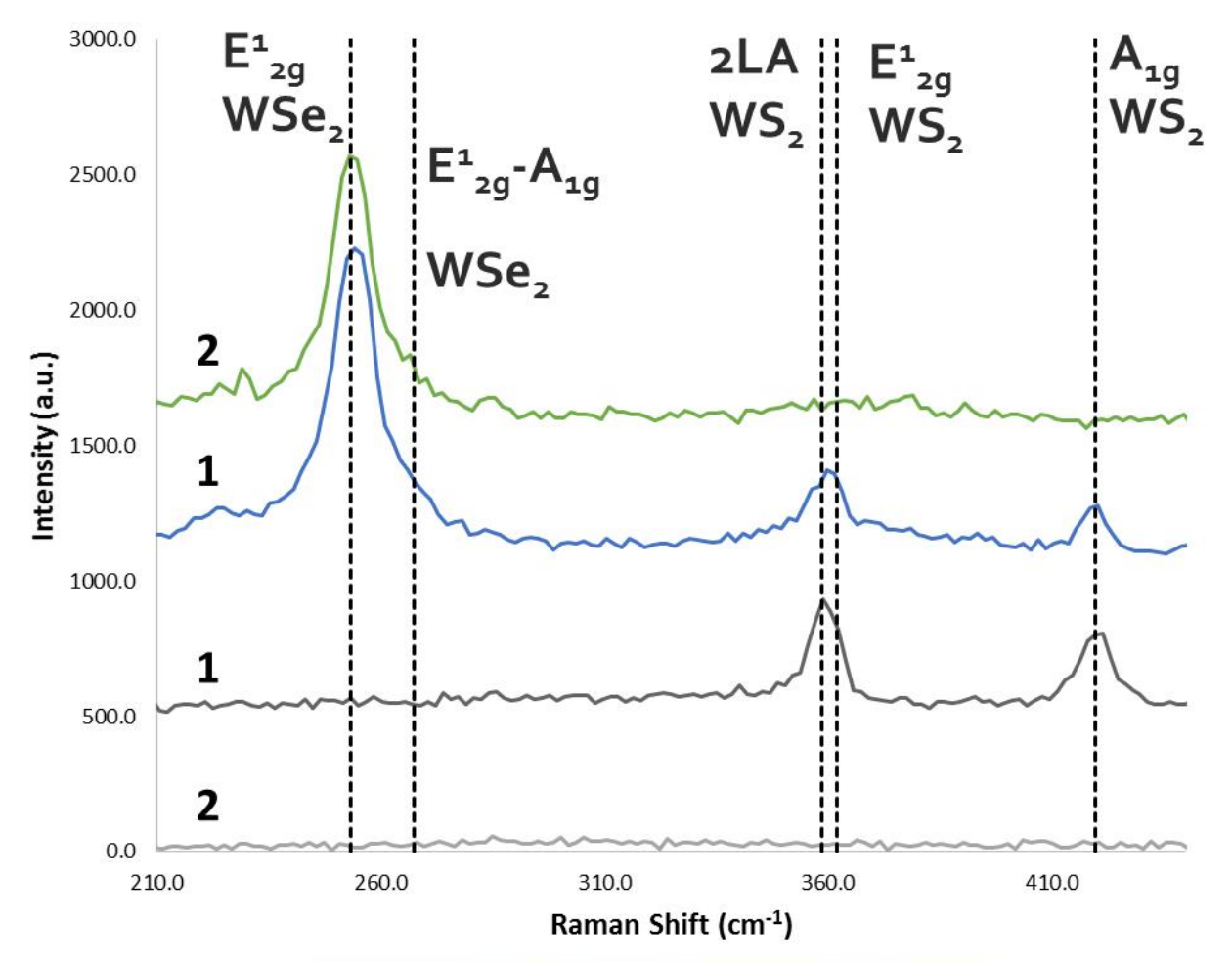

Figure 2.20 scans.

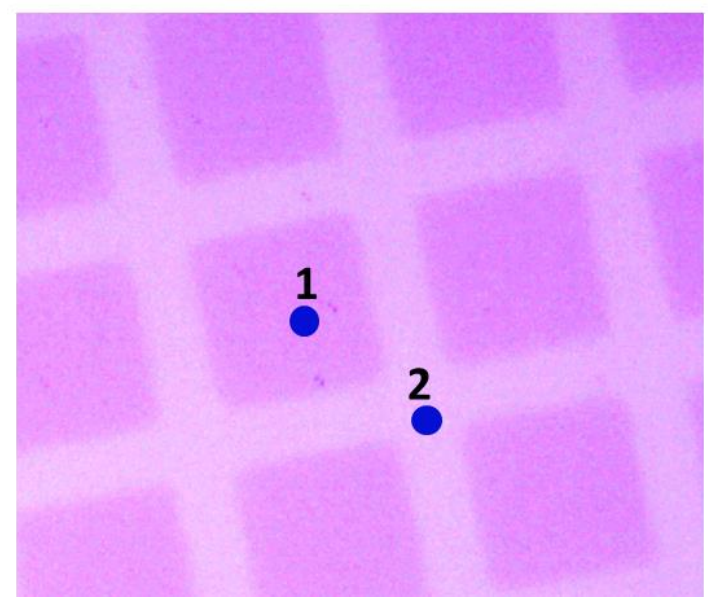

a.) Raman spectrum of bilayer $\mathrm{WS}_{2} / \mathrm{WS}_{2}$ b.) Optical image of locations of raman 


\section{Substrate}

\section{Monolayer $\mathrm{WS}_{2}$}

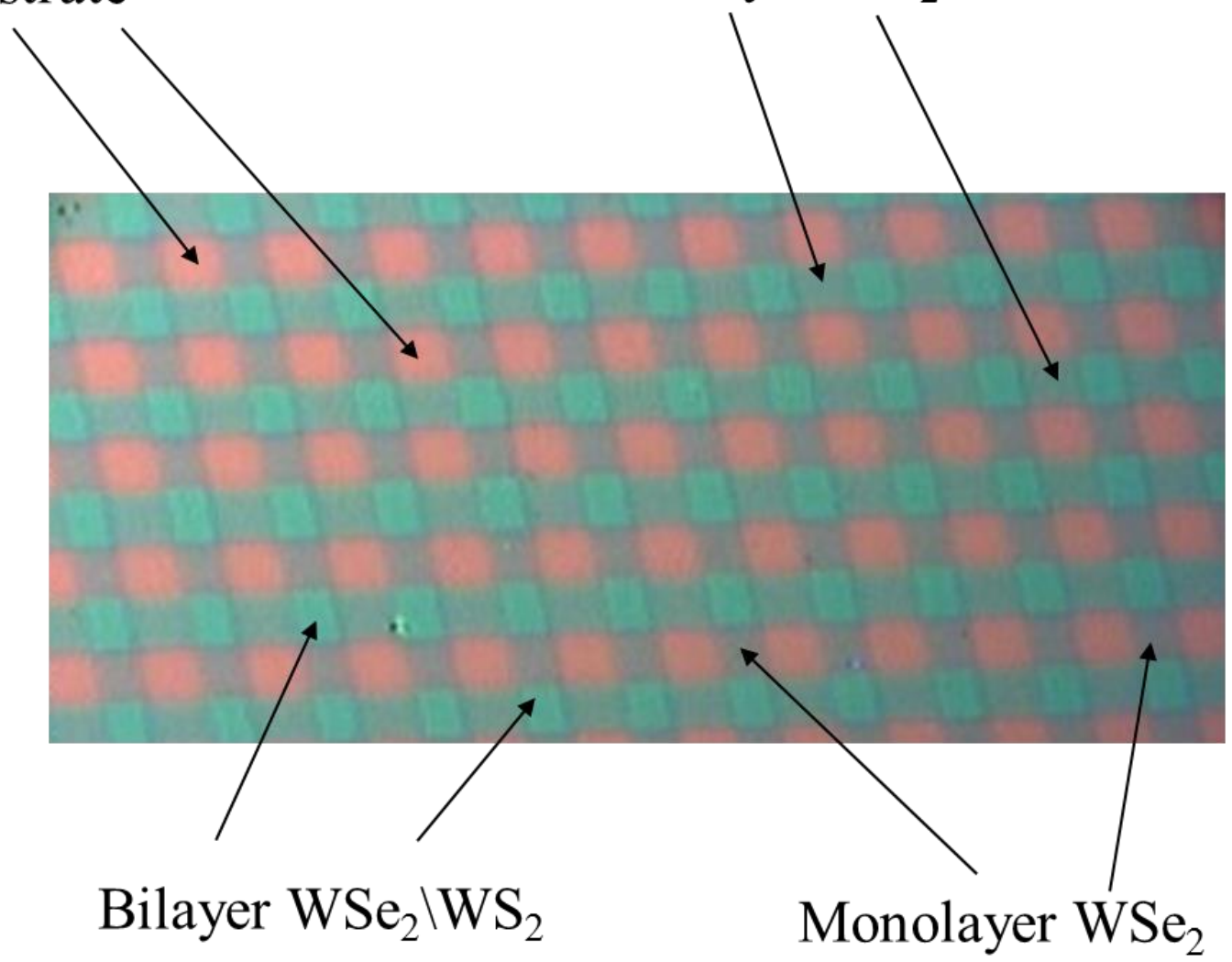

Figure 2.21 Optical image of bilayer $\mathrm{WSe}_{2} / \mathrm{WS}_{2}$ with cross linking pattern

The raman spectrums of Series $\mathrm{B} \mathrm{WSe}_{2} / \mathrm{WS}_{2}$ heterostructure is shown in figure 2.20a. For $\mathrm{WS}_{2}$ the $\mathrm{E}^{1}{ }_{2 \mathrm{~g}}$ and $\mathrm{A}_{1 \mathrm{~g}}$ modes are clearly visible. It also shows precise control over the location of each type of TMD as no signal from $\mathrm{WS}_{2}$ is found in the channel. Very large areas and novel patterns can be fabricated as shown in the optical images in figure 2.21. From raman analysis, it is shown that monolayer based heterostructures can be formed of many different combinations of TMD's while making intricate patterns. 


\subsubsection{Transmission Electron Microscopy}

TEM analysis shown in figures 2.22 , shows the capabilities of this novel method of TMD heterostructure synthesis. TEM data is from Series $\mathrm{A} \mathrm{WS}_{2} / \mathrm{MoS}_{2}$ samples. The sample shown in figure 2.22 was grown under 15 minute chalcogenization time at 800 ${ }^{\circ} \mathrm{C}$. Figures $2.22 \mathrm{a}$ and $2.22 \mathrm{~d}$ respectively show that both inside and outside the squares, these samples are highly polycrystalline. By analyzing (High Resolution TEM combined with Fourier Transform) figure $2.22 \mathrm{~d}$, it is shown that single crystal domains are on the size of 5-15 nm for lateral dimensions. Due to the small single crystal sizes, growth time as well as annealing may be potential routes for increasing film quality. Increasing growth time may also inadvertently increase the possibility of losing the preformed patterns. Figure $2.22 \mathrm{f}$ show that both the top and bottom films adhere to one another and have free standing abilities. TEM cross-section view (Figure 2.22e) over one of the squares, shows that there are between 2-3 atomic layers, which is in excellent agreement with expecting number of layers for stacked structures inside the squares. 

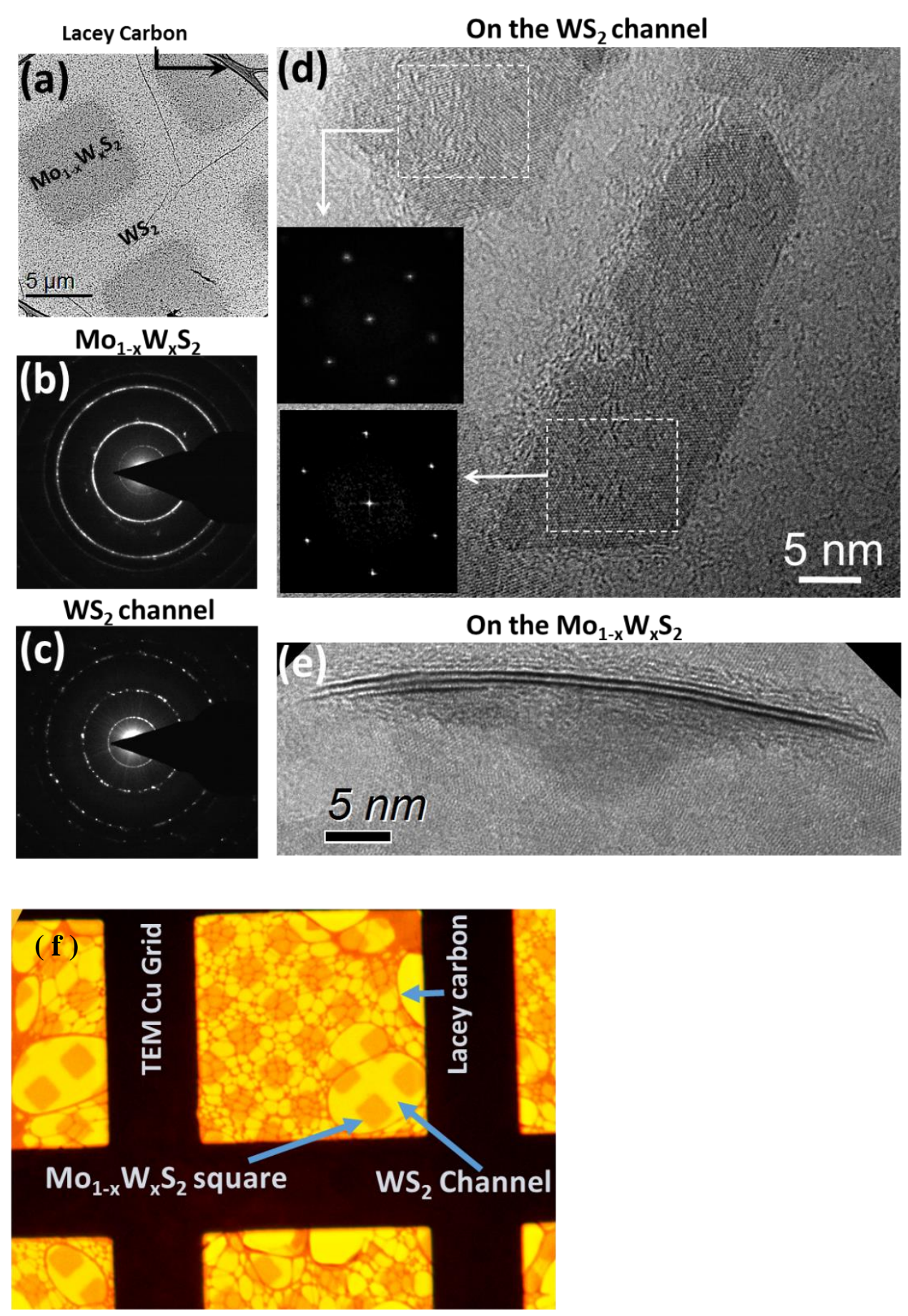

Figure $2.22 \quad$ a.) Low magnification TEM image of a Series-A $W S_{2} / M o S_{2}$ heterostructure. b.) and c.) Electron diffraction patterns in the square and outside the square respectively. (d) HRTEM and FT of nano-crystal domains for 15 min chalcogenization. (e) Cross-section view of the TMD bilayer on the square. $(f)$ Heterostructure transferred to TEM/Lacey Carbon grid 


\subsection{Phosphorene Characterization}

The analysis of phosphorene presents several issues. Stability is of highest concern with phosphorene due to each phosphorus atom in the system having a lone electron pair ready for bonding purposes. Care must be taken, by lowering exposure to ambient conditions.

\subsubsection{Raman Spectroscopy}
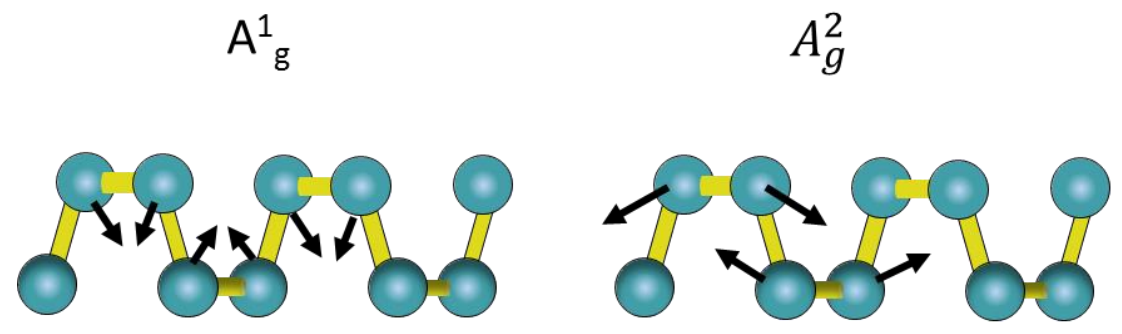

$\mathrm{B}_{2 \mathrm{~g}}$

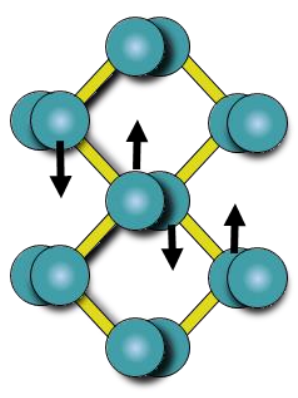

Figure 2.23

Vibrational modes of phosphorene 


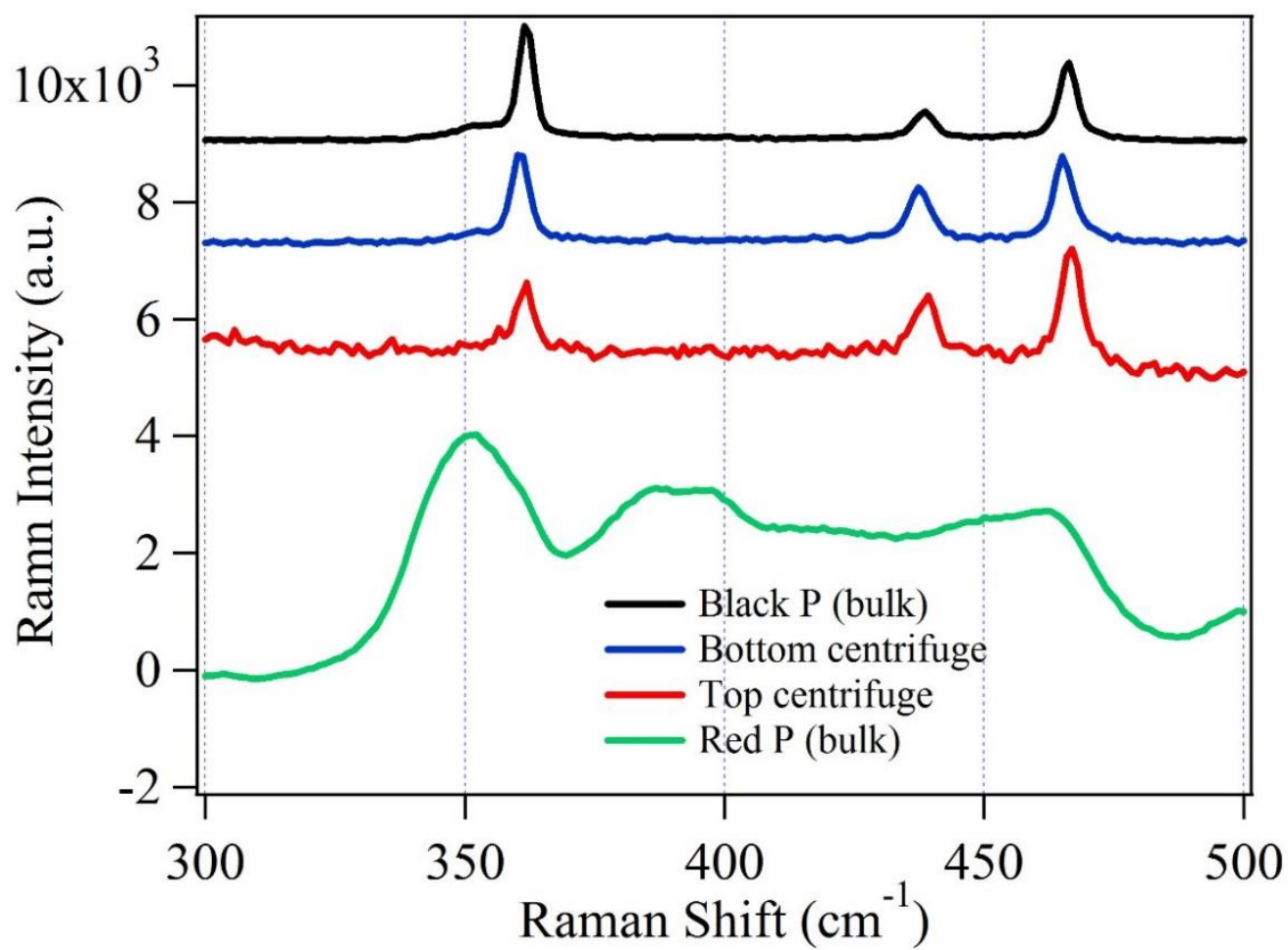

Figure 2.24 Raman spectrums of bulk black phosphorus, bulk red phosphorus, and phosphorene from different locations of centrifuge vial

The vibrational modes of phosphorene are shown in figure 2.23. Main raman modes of phosphorene include the $\mathrm{A}_{\mathrm{g}}^{1}, \mathrm{~B}_{2 \mathrm{~g}}$ and $\mathrm{A}_{\mathrm{g}}^{2}$ modes. The raman spectrums of various setup conditions in LME are shown in figure 2.24. Raman is calibrated to Si 520 $\mathrm{cm}^{-1}$ peak. For Raman, the excitation laser wavelength was $632 \mathrm{~nm}$ at $10 \%$ laser power and exposure time was $15 \mathrm{sec}$ with numerous accumulations to slow degradation of samples. The measured raman peaks are $362.34 \mathrm{~cm}^{-1}, 439 \mathrm{~cm}^{-1}, 467.56 \mathrm{~cm}^{-1}$ for the $\mathrm{A}_{\mathrm{g}}^{1}$, $\mathrm{B}_{2 \mathrm{~g}}$ and $\mathrm{A}_{\mathrm{g}}^{2}$ modes of samples taken from bottom of centrifuge vial. Previous studies have found that the intensity of the $\mathrm{A}_{\mathrm{g}}^{1}$ peak in relation to the Si peak can be used to determine the number of layers of phosphorene [190]. Based on the above intensity ratio calibration, the current work's raman spectrums show between 3 and 9 layers. The 
situation is different for material taken from the bottom of the vial. This spectrum is shown in figure 2.24. Using the aforementioned raman calibration technique, the samples created from material towards the bottom of the glass vial are between 5 and 13 layers. In order to show that no red phosphorus is present in phosphorene, figure 2.24 show a comparison of phosphorene's spectrum to red phosphorus. By comparing the two spectrums it is evident that no red phosphorus can be detected from the phosphorene sample.

\subsubsection{Photoluminescence}

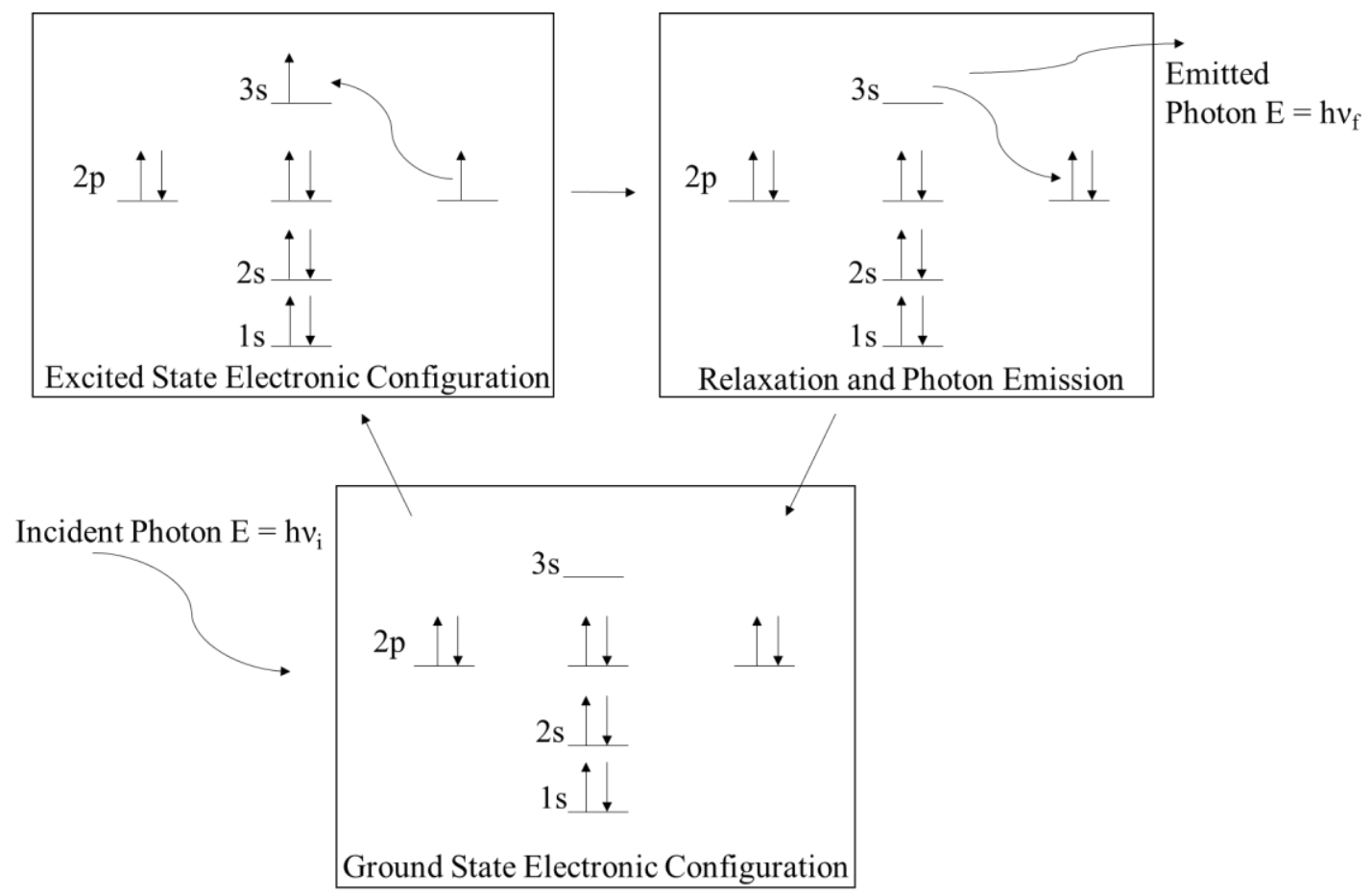


Photoluminescence (PL) process in phosphorene is due to electron-hole interactions. The mechanism that PL is based on is shown in figure 2.25. Incident photons excite electrons in the material. Due to quantum aspects, each photon has a energy that is given by $\mathrm{E}=\mathrm{h} v$, with $\mathrm{h}$ equal to planks constant and $v$ is the frequency of the photon. If the photon has a certain energy, it can promote electrons to higher energy levels. In direct band semiconductors a photon has to have at least the same energy as the materials band gap to induce PL. After the electron has been excited there are several processes that can take place. If the energy of the photon is larger than the energy band gap, the electron will be excited deep into the conduction band of the material. Following this excitation, the electron can experience non-radiative relaxation by creating phonons. However, once it reaches the conduction band minimum, the electron can undergo radiative relaxation by excitonic decay. Energy is released from the excitonic decay in the form of a photon with energy equal to the lower bound of the energy band gap of the semiconductor. Phosphorene has been thoroughly studied through the use of photoluminescence [191]. Due to PL being dependent on the band gap of the material, it is especially useful in the study of phosphorene. As mentioned in previous sections, the band gap of phosphorene increases as the number of layers of phosphorene decrease. Since PL depends directly on the band gap, a calibration method has been develop to identify the number of layers based on PL emission spectrums [191]. Figure 2.26 shows the PL of bulk black phosphorus and phosphorene samples taken from the top and bottom of the sample after centrifugation. 


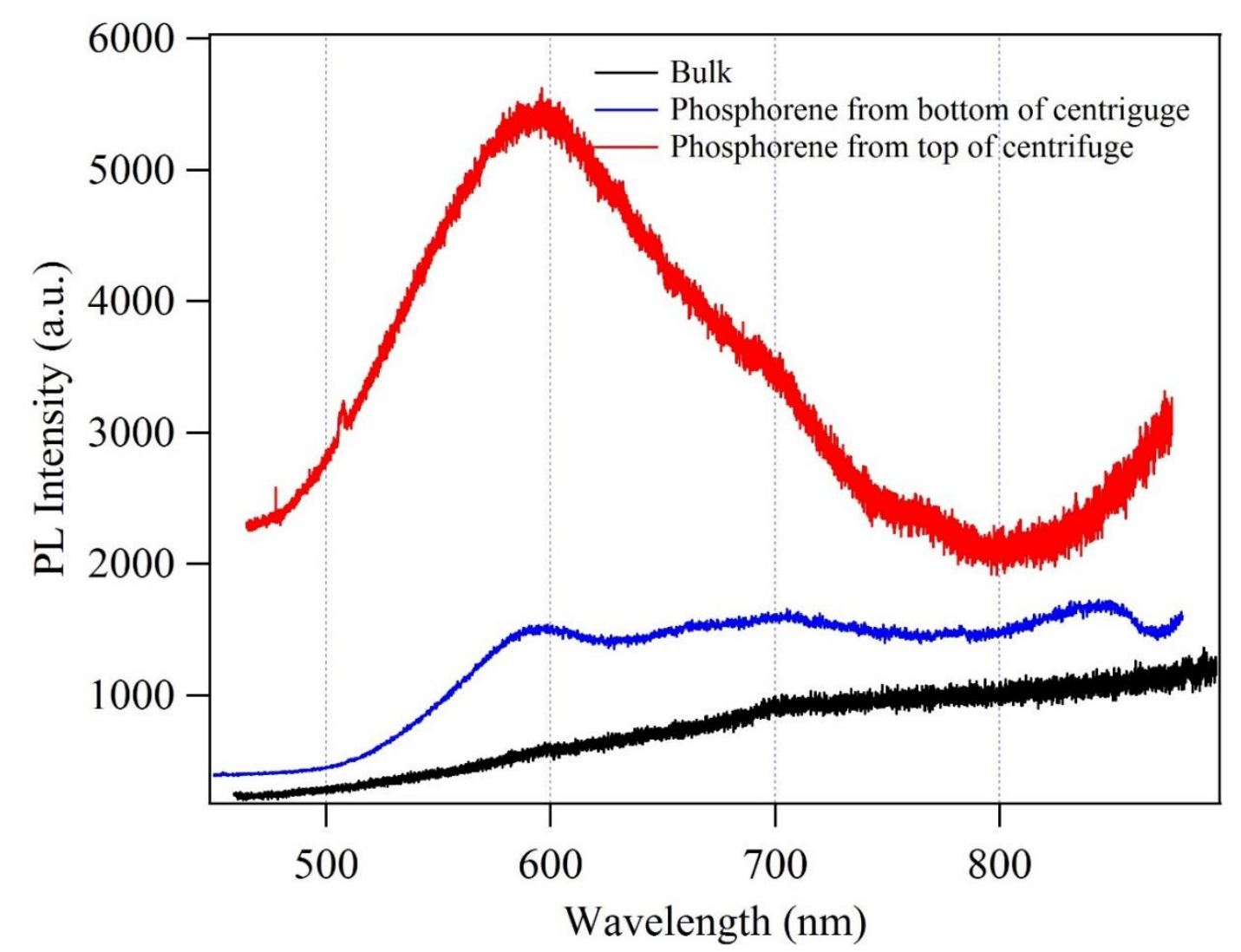

Figure 2.26 PL from bulk black phosphorus and phosphorene from different locations of the centrifuge vial

Photoluminescence was done using $442 \mathrm{~nm}$ wavelength excitation laser at $10 \%$ and 30 second exposure time. Photoluminescence (figure 2.26) shows that for bulk black phosphorus no peaks are present in the region of interest. This is expected due to the band gap of bulk black phosphorus being in the infrared region with a value of $E_{g}=0.3 \mathrm{eV}$ [192]. The samples that had the smallest layering number (taken from top of centrifuge vial) have a very strong peak at $593.28 \mathrm{~nm}(2.09 \mathrm{eV})$ and weaker peaks at $696.00 \mathrm{~nm}$ $(1.78 \mathrm{eV})$ and $769.00 \mathrm{~nm}(1.61 \mathrm{eV})$. The weaker peaks are believed to be due to none uniform distribution of layering number across the substrate. Non-uniformity has been found to be a common issue with liquid mechanical exfoliated samples [146]. The 
strongest peak at $2.09 \mathrm{eV}$, is suspected to be due to monolayer phosphorene. Although PL emission is directly related to the band gap, it is actually an approximation to the lower bound of the energy band gap. Excited elections are not entirely free (as long as the excitation source is not strong enough to cause electron ejection). Once electrons are excited they leave behind a hole. The electron-hole interaction slightly lowers the energy of the subsequent emitted photons. Therefore the actual band gap may be larger than 2.09 $\mathrm{eV}$. Energy band gaps of $1.6 \mathrm{eV}$ [190] and $1.29 \mathrm{eV} \mathrm{[185]} \mathrm{have} \mathrm{been} \mathrm{found} \mathrm{for} \mathrm{two} \mathrm{layer}$ phosphorene from photoluminescence estimates, which is smaller than the value found in the current work for single layer phosphorene. The calculated band gap for single layer phosphorene is $2 \mathrm{eV}$ [193] and is close to the current works value of $2.09 \mathrm{eV}$.

\subsubsection{Scanning Electron Microscopy}
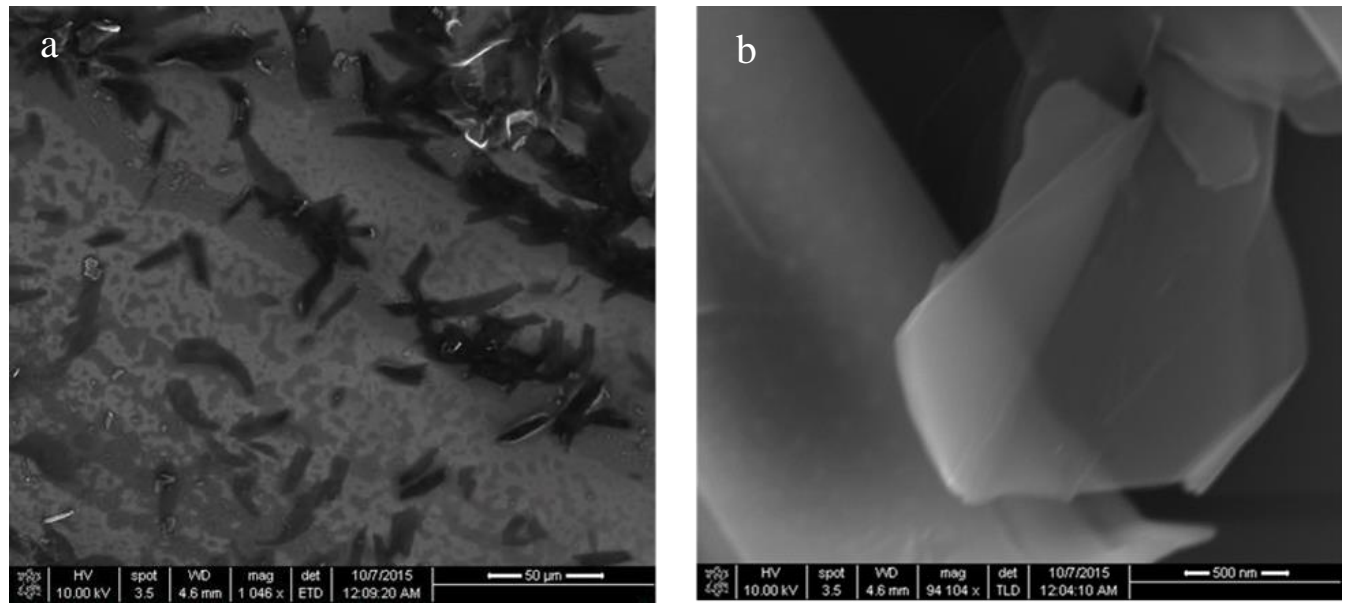

Figure 2.27

a.) and b.) SEM images of phosphorene 
Topological studies of phosphorene were done using scanning electron microscopy (SEM). Figure 2.27 is from a sample of phosphorene that was obtained from the top of the centrifuge vial after the LME process was completed. Black flakes seen on the $\mathrm{SiO}_{2} / \mathrm{Si}$ substrate are phosphorene. Non-uniform distribution of phosphorene across the substrate is seen. It appears that as the solvent is drying, the phosphorene flakes group together in close proximity. There are areas on the substrate that have higher concentrations of phosphorene, while others have no visible flakes. Non-uniformity is also seen in figure 2.27b. Multiple phosphorene flakes can be seen overlapping one another. This agrees with PL studies in previous sections, which show multiple PL peaks associated with several different layering amounts in the same location. Overlapping shown in SEM confirms that LME can indeed have issues with sample distribution in regards to layering number.

\subsubsection{Transmission Electron Microscopy}

Transmission Electron Microscopy (TEM) analysis is shown in figure 2.28b. Phosphorene flakes were found to be $2 \times 6 \mu \mathrm{m}$ in lateral dimensions. These samples are made by suspending a TEM grid into the dimethlyformalhide/phosphorene mixture after LME. The TEM grid also had lacey carbon as a structural support material. As the grid was slowly pulled out of the mixture, it was able to catch several flakes. Once the grid/phosphorene system had dried, it was placed inside the transmission electron microscope. Due to the presence of less material as the solvent dried, in addition to a lack of continuous substrate, the flakes found for the TEM study are somewhat smaller than the flakes formed by drop casting onto $\mathrm{SiO} 2 / \mathrm{Si}$ substrates. 

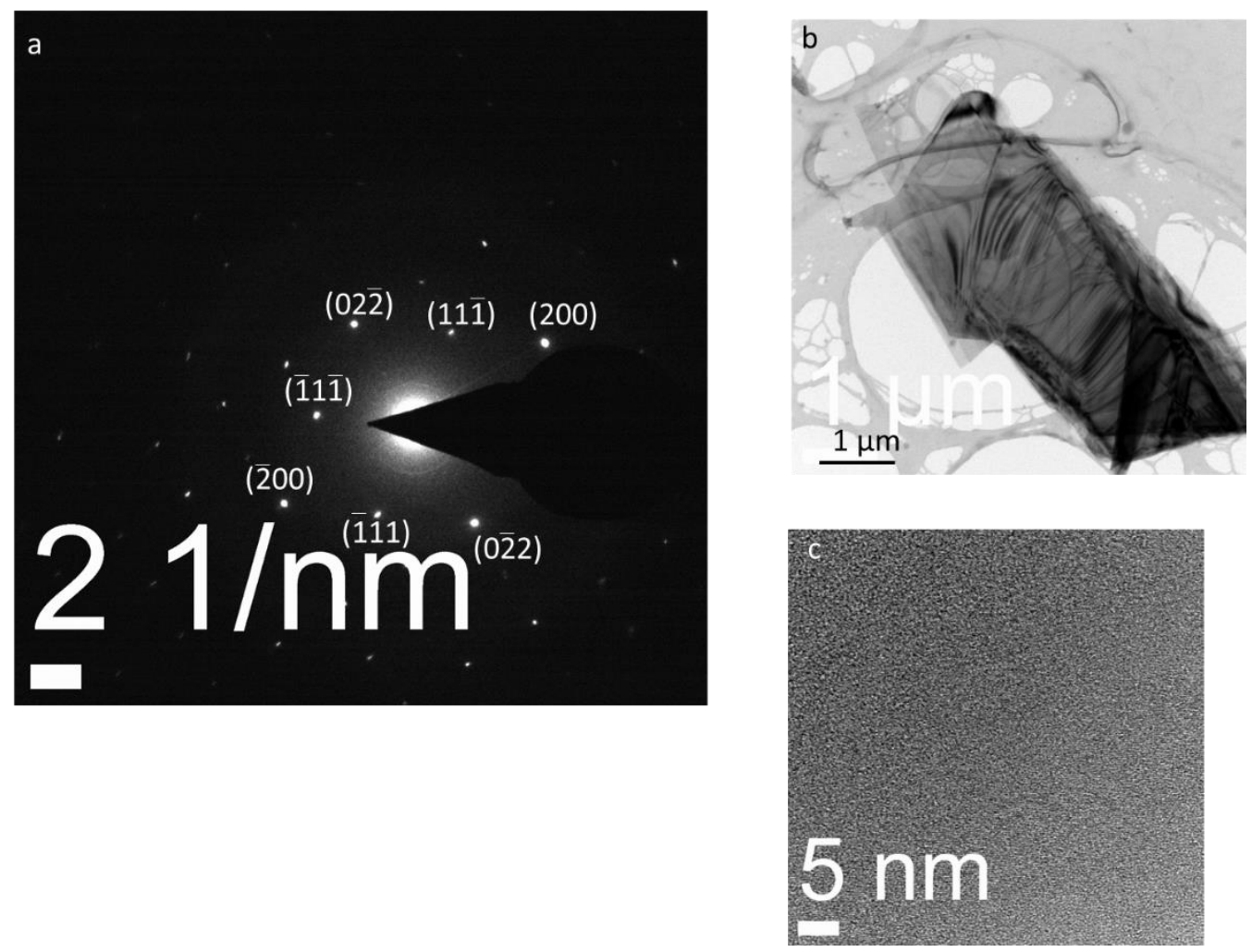

Figure 2.28

a.) Electron diffraction of phosphorene. b.) TEM of phosphorene c.) HR-TEM of phosphorene

Figure $2.28 \mathrm{~b}$ shows that folds are formed in the phosphorene flakes and that it has free standing capabilities. The aforementioned folds may be a consequence of the drying process of the solvent. HR-TEM (high resolution) is shown in figure $2.28 \mathrm{c}$ on a few layer sample. It has been found that few layer phosphorene is beam sensitive for HR-TEM [190] and this is confirmed in figure $2.28 \mathrm{c}$ as beam induced breakdown of the structure of phosphorene atoms. As the HR-TEM scan was being done, the degradation of phosphorene could be easily seen. Due to the degradation, all HR-TEM based analysis was done on a separate flake of phosphorene. Electron diffraction shows that the material is single crystal in figure $2.28 \mathrm{a}$, due to no spreading of the diffraction pattern due to multiple orientated crystals. The experimentally found d-spacing's are found to be 0.261 
$\mathrm{nm}, 0.161 \mathrm{~nm}$, and $0.174 \mathrm{~nm}$ for planes (111), (200), and (022) respectively. Previous findings found $0.26 \mathrm{~nm}$ for the (111) plane [146] and $0.1997 \mathrm{~nm}$ for (022) plane [194]. For the current work the (010) plane is the zone axis. Energy dispersive x-ray spectroscopy (EDX) was also done using the TEM setup. EDX shows that phosphorus is the main chemical element (figure 2.29). There are trace amounts of carbon and oxygen. Oxygen may be due to environmental effects, and carbon can be attributed to both environmental effects and potential doping from the Dimethyl Formaldehyde solvent.
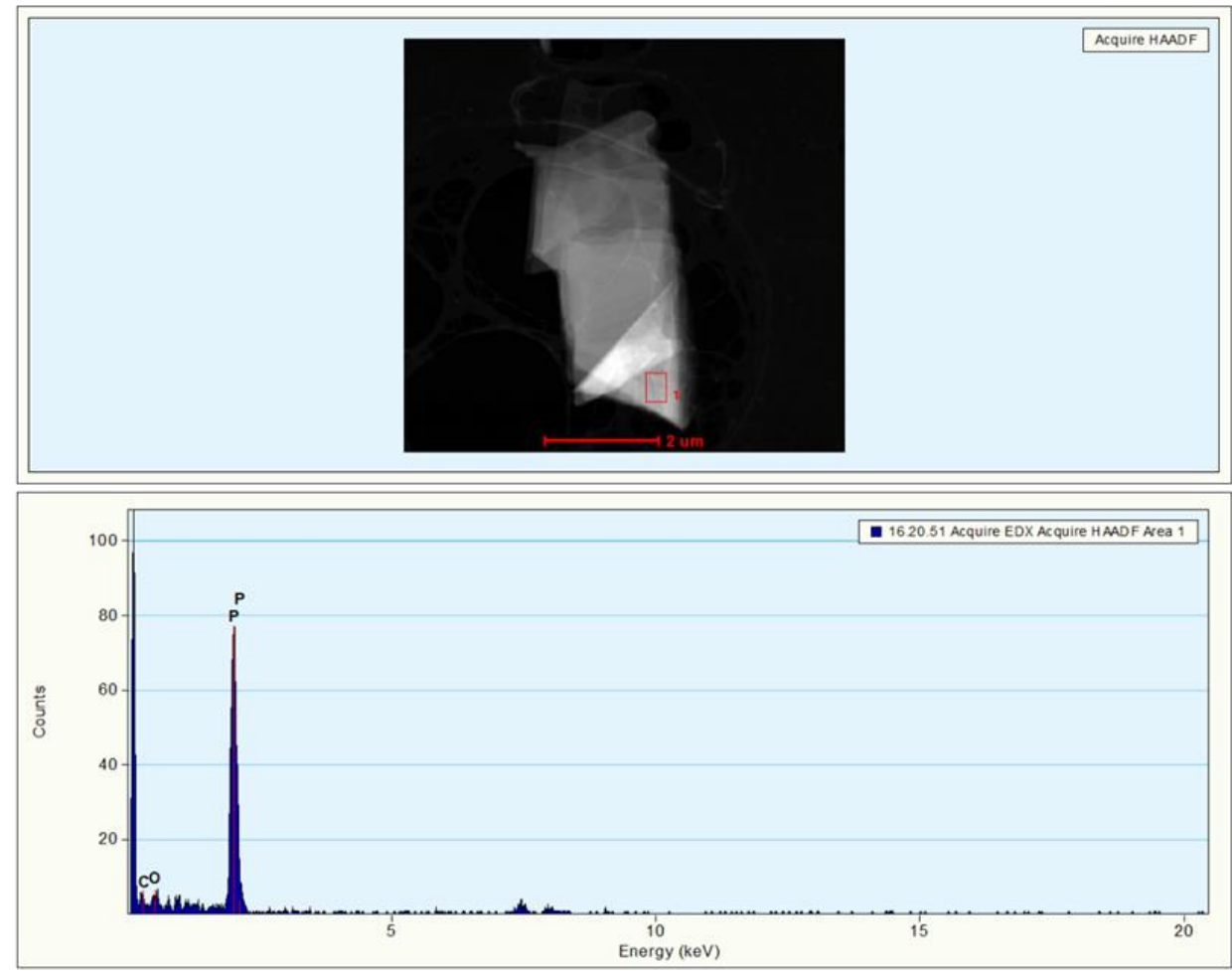

Figure 2.29 EDX of phosphorene 


\subsubsection{Atomic Force Microscopy}
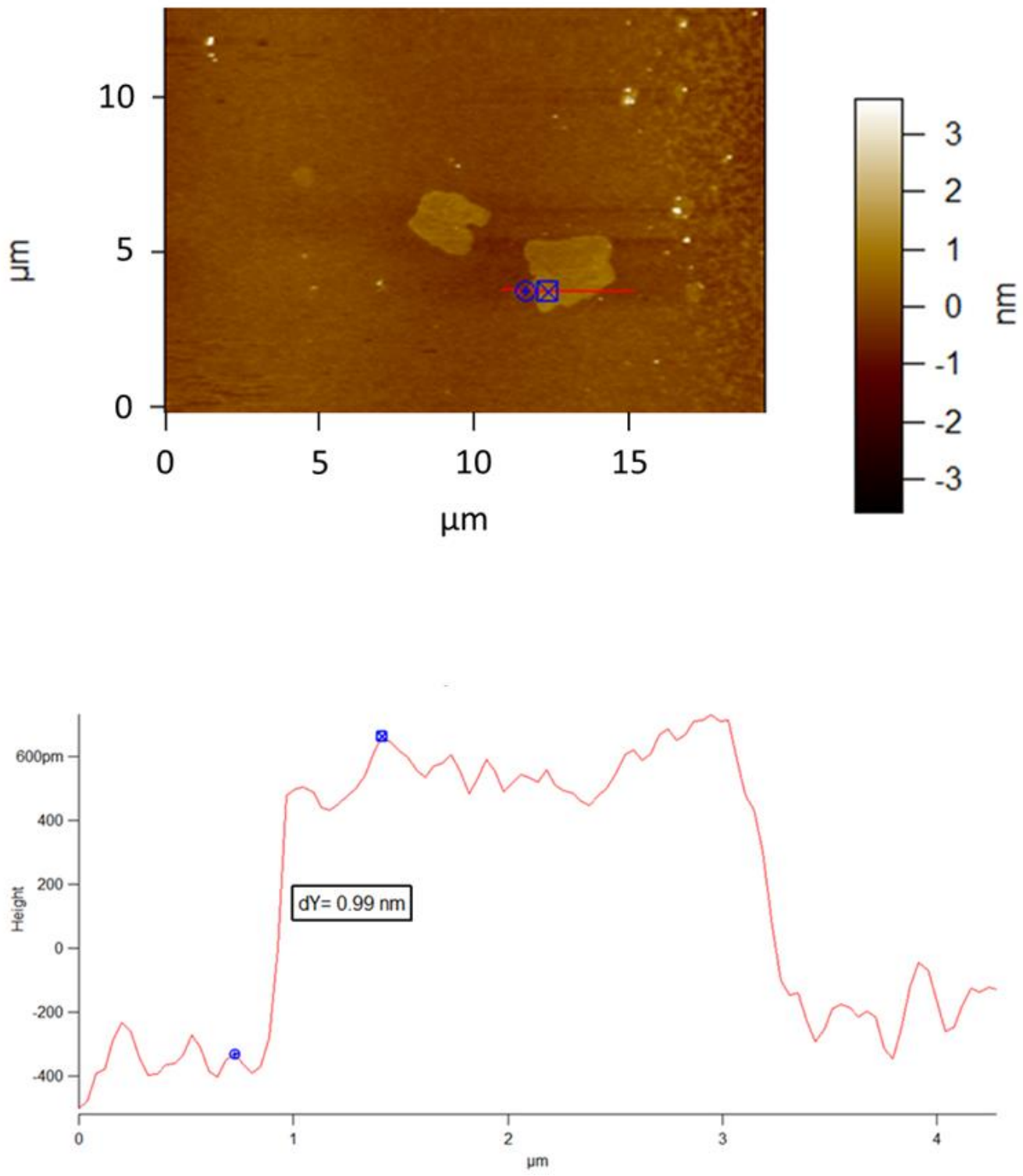

Figure 2.30 AFM of phosphorene 
Atomic Force Microscopy is a technique that is extremely well suited for the analysis of 2D materials. Previous research has shown that phosphorene has a step height of $0.85 \mathrm{~nm}$ [195]. Using tapping mode in AFM, the current work has found a step height of $0.99 \mathrm{~nm}$ for single layer phosphorene. The AFM height profile is shown in figure 2.30. In addition the three dimensional rendering of the height profile is showed in figure 2.31. Across the surface of the phosphorene flake the height is very uniform.

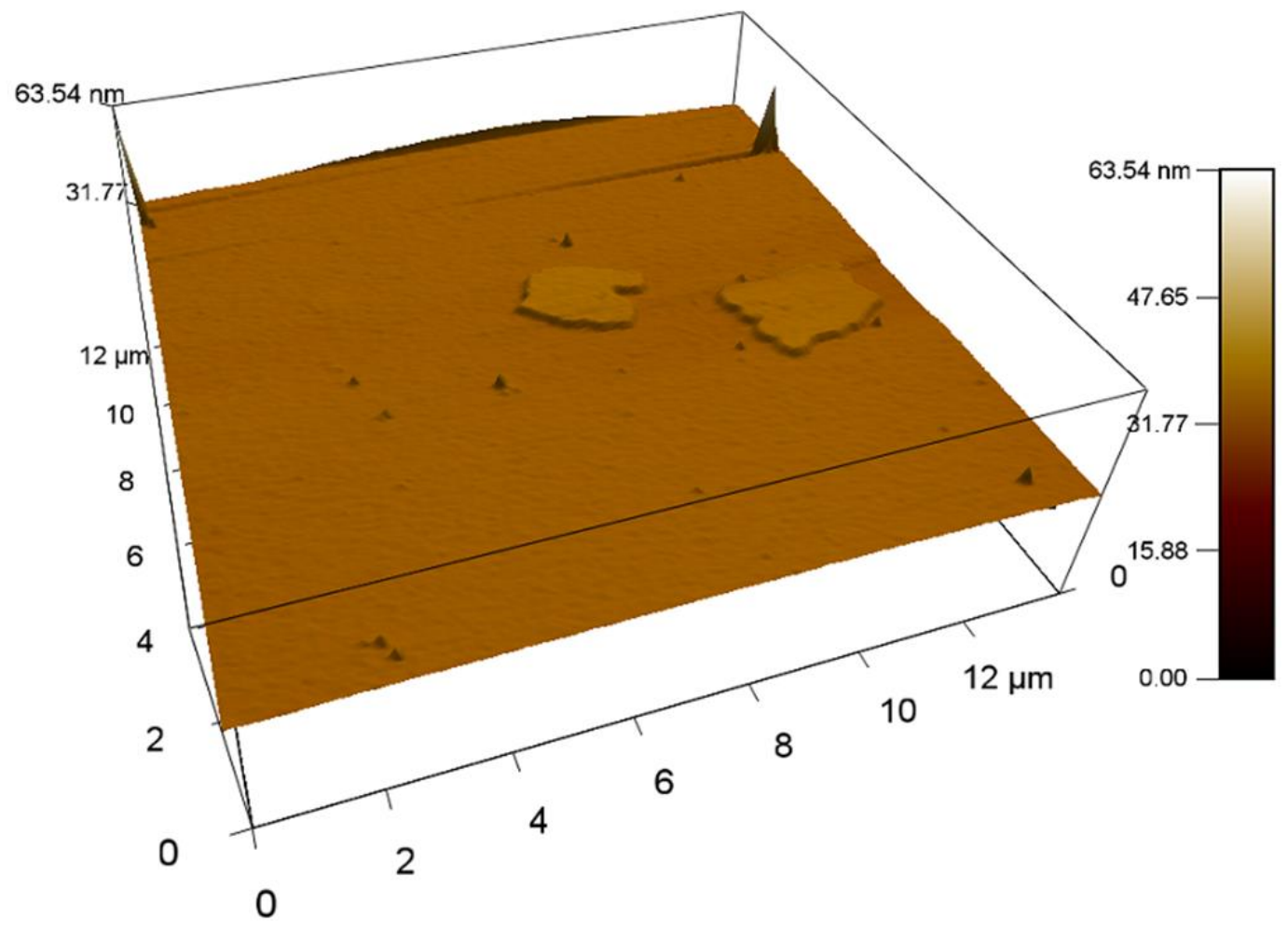

Figure 2.31 3D rendered AFM of phosphorene 


\section{CHAPTER 3 - PHOSPHORENE BASED OPTOELECTRONIC DEVICES}

\subsection{Introduction}

Optoelectronic devices are electronic devices that experience light induced property changes. They include photodetectors, photovoltaics and light emitting diodes (LED). Optoelectronic devices have been around for many years. Complimentary to optoelectronic devices are field effect transistors. Field effect transistors are one of the most fundamental and important electronic devices ever created. Field effect transistors (FET) are the back bone of modern day computing. FET designs can be used to add more functionality to optoelectronic devices. FET's are used in integrated circuits, op-amp circuits, and as switches. The FET setup is also used in other areas such as gas sensors.

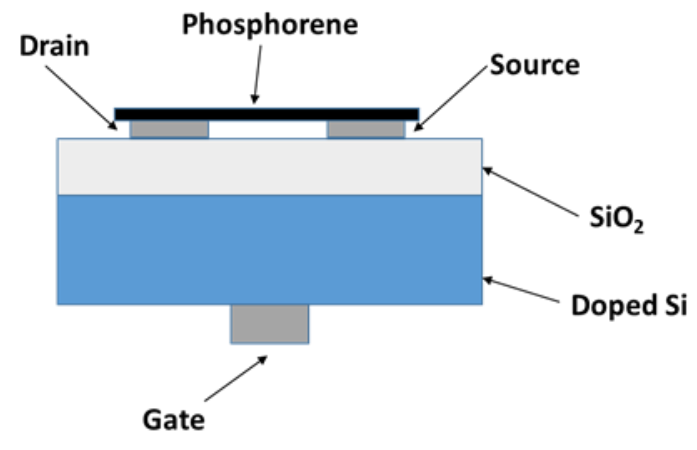

Figure 3.1: $\quad$ Schematic of field effect transistor 
A FET is an electronic device that has unipolar (one dominate carrier type) conductivity. This conductivity is enhanced or quenched by altering electric fields in the device. Figure 3.1 shows a schematic of a back-gated phosphorene FET. A FET consists of drain, source, and gate contacts. The drain-source contacts are connected (conducting channel) by controlling an electric field caused by applying a voltage between the gatesource contacts. There is a dielectric material positioned between the gate contact and the other electrodes. FET's whose conducting channel has electrons as its primary charge carrier, has n-type conductivity. If holes are dominate, then it has p-type conductivity.

Moore's law states that the maximum number of transistors in an integrated circuit doubles every two years. The method of keeping up with Moore's statement is by scaling electronics to smaller and smaller sizes. Graphene opened the ultimate method of electronic scaling. 2D and 1D materials are currently the smallest scale upon which known materials can be fabricated. As such, implementing 2D materials is one way of producing more transistors in smaller areas. Limits of current silicon based technology is another motivation for 2D materials [196]. Graphene was integrated into a heterostructure waveguide, which could detect infrared radiation [197]. The amount of information that has been developed in relation graphene is staggering. However, graphene does has limitations. As such, many processes such as doping, heterostructures, and surface functionalization techniques have been developed to make 2D devices. This includes using new 2D materials in devices such as TMD's and phosphorene.

The key aspects of FET's are its transfer characteristics, I-V properties, charge carrier mobility, conductivity type, temperature dependence, and environmental based performance. Transfer characteristics is also known as gate dependence. As the gate 
voltage $V_{g}$ is varied, the drain-source voltage $V_{d s}$ is held constant, and drain-source current $\mathrm{I}_{\mathrm{ds}}$ is measured. One of the first properties that can be extracted from the transfer curves, is the conductivity type of the conducting channel material. Increasingly positive $\mathrm{V}_{\mathrm{g}}$ that results in increased drain-source current, the material is n-type. As $\mathrm{V}_{\mathrm{g}}$ becomes more positive, it populates the dielectric material with holes (figure 3.2). These holes attract electrons in the conducting material, which increases electrons in the conducting channel. This increase in electrons is followed by an increase in $\mathrm{I}_{\mathrm{ds}}$ due to electrons being the dominate charge carrier. The reason they behave as n-type is due to their fermi level being closer to the conduction band minimum. In the case of p-type FET's, the fermi level is closer to the valence band maximum. Transfer characteristics for a p-type material is different than that of n-type material. For increasing positive $V_{g}, I_{d s}$ decreases. The reason is that a negative $\mathrm{V}_{\mathrm{g}}$ is needed to increase the charge carriers (holes) in the conducting material, if one wants to increase current levels. However, a positive $\mathrm{V}_{\mathrm{g}}$ cannot attract holes (they repel), as such the conducting channel is effectively pinched off. A region is formed inside of the conducting channel that contains virtually no charge carriers. This region is defined as the depletion region. Figure 3.2 shows a FET with ptype conducting channel in both reverse-biased (figure 3.2a) and forward-biased (figure 3.2b) setups. 

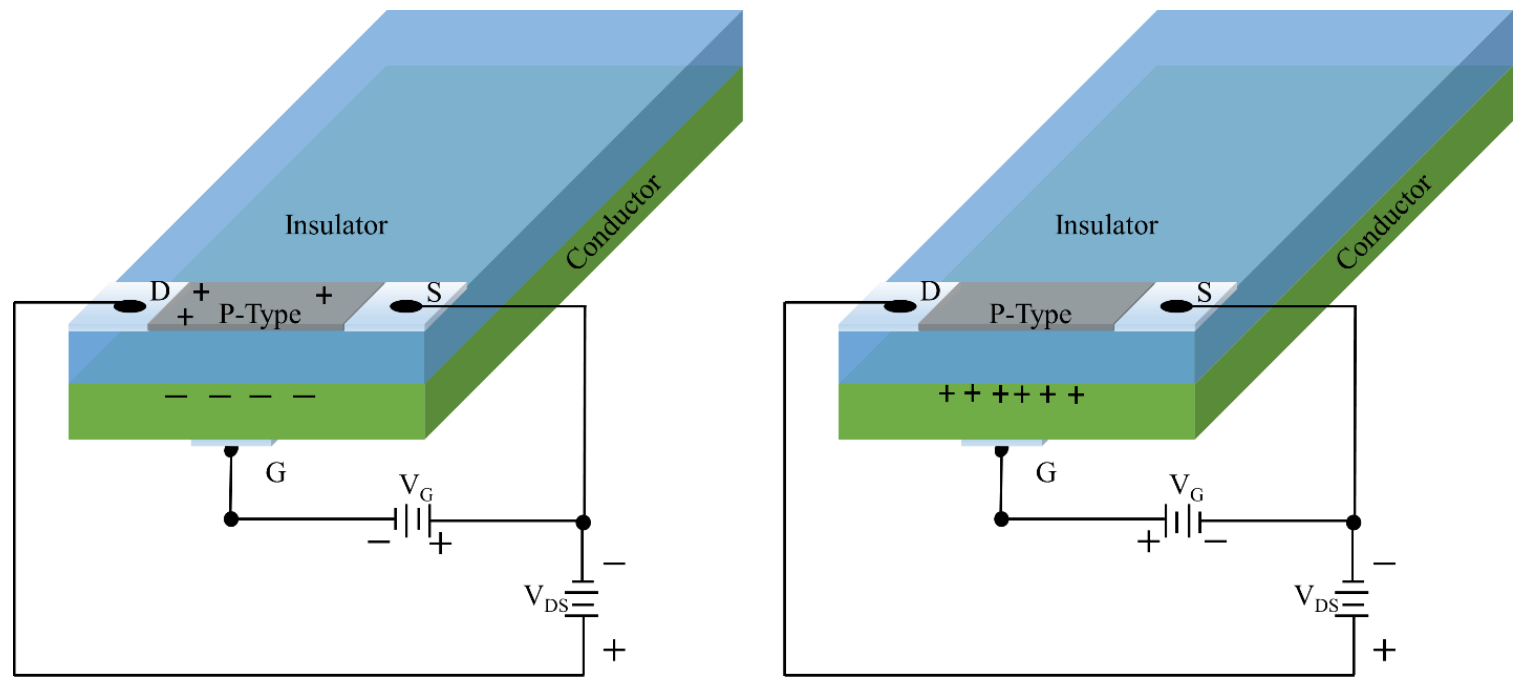

Figure 3.2:

P-type field effect transistor a.) with reverse-biased and $b$.) forward-biased

Pinch off voltage is defined as the voltage $V_{g}$, that makes $I_{d s}$ approach zero. This is another property that can be determined by transfer characteristics. One of the most important properties that can be derived from the transfer characteristics is mobility. Mobility is a measure of how rapidly a charge carrier can travel through a material. Both electrons and holes have motilities. Higher motilities allow for FET's to be used in high speed switching applications. Both electron and hole motilities are determined from the conventional transconductance equation $\mu_{a}=\left(d I_{d s} / d V_{g}\right)(L / W)\left[\left(C_{o x} * V_{g}\right)^{-1}\right]$.

Variables $\mathrm{L}$ and $\mathrm{W}$ are channel length and width respectively. $\mathrm{C}_{\mathrm{ox}}$ is the capacitance of the gate oxide and defined as $\mathrm{C}_{\mathrm{ox}}=\varepsilon_{\mathrm{ox}} / \mathrm{d}_{\mathrm{ox}}[87,88]$. The gate oxide thickness is $\mathrm{d}_{\mathrm{ox}}$ and $\varepsilon_{\mathrm{ox}}$ is the dielectric constant. $\mathrm{I}-\mathrm{V}$ curves are similar to gate dependence, however now $\mathrm{V}_{\mathrm{g}}$ is held constant and $V_{d s}$ is allowed to vary while $I_{d s}$ is measured. From the $\mathrm{I}-\mathrm{V}$ curve there are various regions of interest. Two of these important regions are the ohmic region and 
saturation region. The ohmic region, is the area in which the $\mathrm{I}-\mathrm{V}$ curve behaves in a linear fashion. In the ohmic region, the I-V curve follows ohms law,

$$
V=I R
$$

with I and R being current and resistance respectively.

Saturation region is the region in which $\mathrm{I}_{\mathrm{ds}}$ stabilizes near a certain value $\left(\mathrm{I}_{\mathrm{ds}}(\mathrm{sat})\right.$ or saturation current). The voltage at which the I-V curve crosses over into the saturation region is defined as the saturation voltage,

$$
V_{d s}(s a t)=V_{g}-V_{T}
$$

$\mathrm{V}_{\mathrm{T}}$ is the threshold voltage and is the minimum gate voltage needed to open the conduction channel between source and drain contacts. There are two more regions of interest. The breakdown region is the point at which $\mathrm{V}_{\mathrm{ds}}$ becomes larger then the conducting channel can withstand. As such any small increases in $\mathrm{V}_{\mathrm{ds}}$ result in much more extreme changes in $I_{d s}$. The other region is called the avalanche region. In the avalanche region, the electric fields in the FET become strong enough to rip electrons from the atoms in the conducting channel. This leads to further increases in current and potential sample damage.

The environment that real world devices operate in have varying temperatures and atmospheric conditions. Real world devices operate in vary demanding environments. Even though temperatures can be regulated in a computer through the use of heat sinks, fans, and liquid cooling, other effects such as moisture and gas particles are harder to account for. The interaction of FET's with atoms such as oxygen and water can alter device performance. Some atoms behave as donors of electrons or acceptors of electrons. 
Even light can increase or decrease current values. These varying changes are the basis of many photodetectors and gas sensors. With phosphorene having such notorious stability issues, understanding its device performance in various environments is key.

\subsection{Transfer Characteristics of Phosphorene FET}

The mobility of phosphorene various from 12 to $1350 \mathrm{~cm}^{2} / \mathrm{Vs}$ [198]. These large variations are due to different synthesis techniques, device design, dielectric material, along with not listed factors such as laboratory conditions that the study was done under. Phosphorene topped with a capping layer of $\mathrm{Al}_{2} \mathrm{O}_{3}$, was found to have mobility of 95.6 $\mathrm{cm}^{2} / \mathrm{Vs}$ [118]. Capping layers are found to slow degradation effects and maintain mobility properties of phosphorene [198]. Conductivity of phosphorene has been shown to be p-type [90, 199-201]. It must also not be forgotten that monolayer phosphorene has a $2 \mathrm{eV}$ band gap which is in the visible region with $\lambda=619.5 \mathrm{~nm}$. This means that for phosphorene based FET's, light absorption can alter properties by optically exciting electrons. In order to effectively utilize phosphorene as a FET, the ways in which gases and light sources effect it, must be know. 
3.3 In-situ environment dependent transport in phosphorene

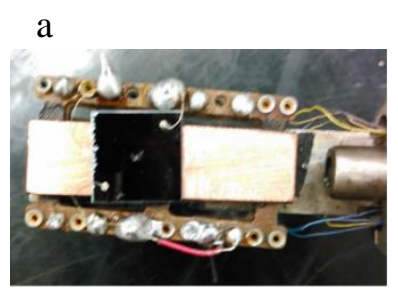

b

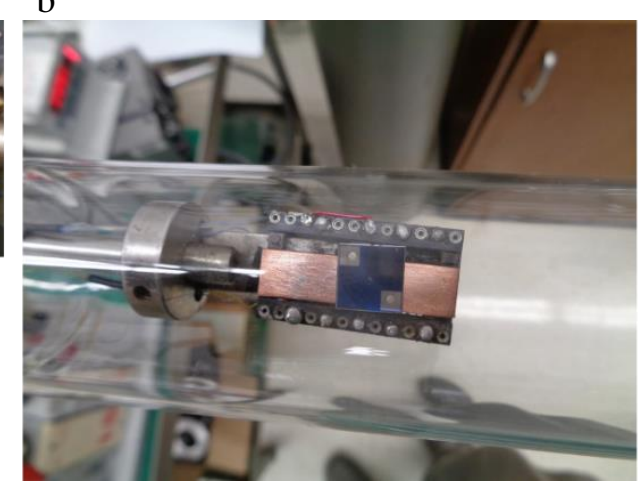

d

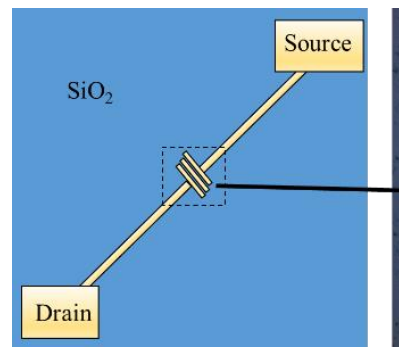

$\mathrm{c}$

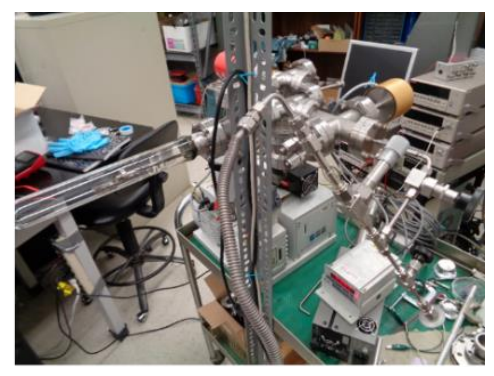

e

Figure 3.3: $\quad$ a.) Phosphorene FET on probe. b.) Phosphorene FET inside vacuum system. c.) In-situ setup. d.) Electrode pattern on chip

Figure 3.3 shows the setup used to do the in-situ study. The system is a custom vacuum chamber that can reach down to 6.5 Torr. Phosphorene is drop cast onto Al contacts that have previously been deposited on $\mathrm{SiO}_{2} / \mathrm{Si}$ substrates. Figure 3.3a shows one sample. The sample is fixed to a probe that can withstand elevated temperatures. There are electrical feed ports that allow for in-situ measurements while maintaining a sealed environment. Transfer characteristics are measured using Keithley picometer/voltage source meter system to control the measurements. The in-situ system also allows for the attachment of various light emitting diodes that can be attached on the probe directly next to the phosphorene. This setup allows for the phosphorene FET to be 
placed in a controlled environment, and exposed to different light sources while minimizing external light sources. The system is also capable of heating the sample through the use of a quartz tube furnace. Once the phosphorene sample is in the vacuum system, it sits in a high temperature quartz glass tube that allows the heat from the tube furnace to be transferred to the sample in the closed environment. Lastly, the vacuum system has ports that allow for different gases to be introduced to the phosphorene sample while keeping exposure to ambient conditions to a minimum.

\subsubsection{Gas Interaction}

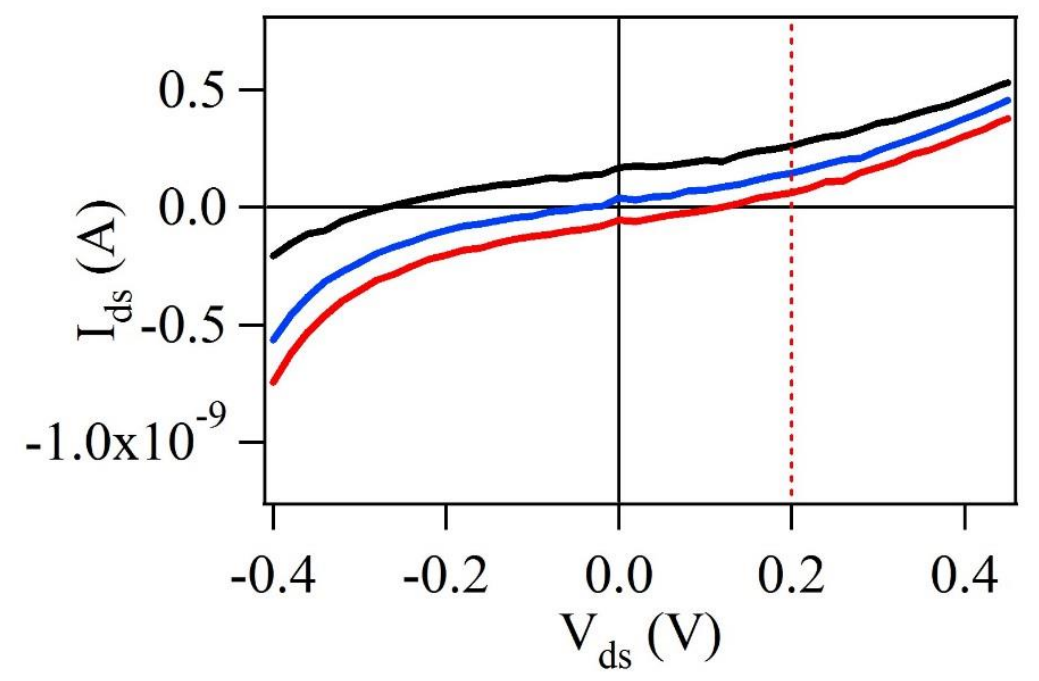

Figure 3.4:

Phosphorene fet I-V curves for $\mathrm{Vg}=20 \mathrm{~V}$ (black), $0 \mathrm{~V}$ (blue), and $-10 \mathrm{~V}$ (red)

A two-probe setup is employed, which means resistance effects of the contact material is included in the data. When phosphorene was drop cast on the contacts, a dc bias of $0.2 \mathrm{~V}$ was applied to attract the flakes as the DMF dries. Typical I-V curve of 
phosphorene is shown in figure 3.4 for gate voltages $\mathrm{V}_{\mathrm{g}}=-10 \mathrm{~V}, 0 \mathrm{~V}$, and $20 \mathrm{~V}$ in air. Breakdown regions begin below $-0.5 \mathrm{~V}$ and above $0.5 \mathrm{~V}$. For this reason further analysis was done at smaller drain-source $\left(\mathrm{V}_{\mathrm{ds}}\right)$ voltages to avoid damage. Comparing valves of $\mathrm{I}_{\mathrm{ds}}$ (drain-source current) for $\mathrm{V}_{\mathrm{g}}$ equal to $0 \mathrm{~V}$ and $20 \mathrm{~V}$ shows increases in current of about $0.05 \mathrm{~A}$ at $\mathrm{V}_{\mathrm{ds}}=0.2 \mathrm{~V}$. It is noted that the samples display characteristic non-linear semiconducting I-V curves.

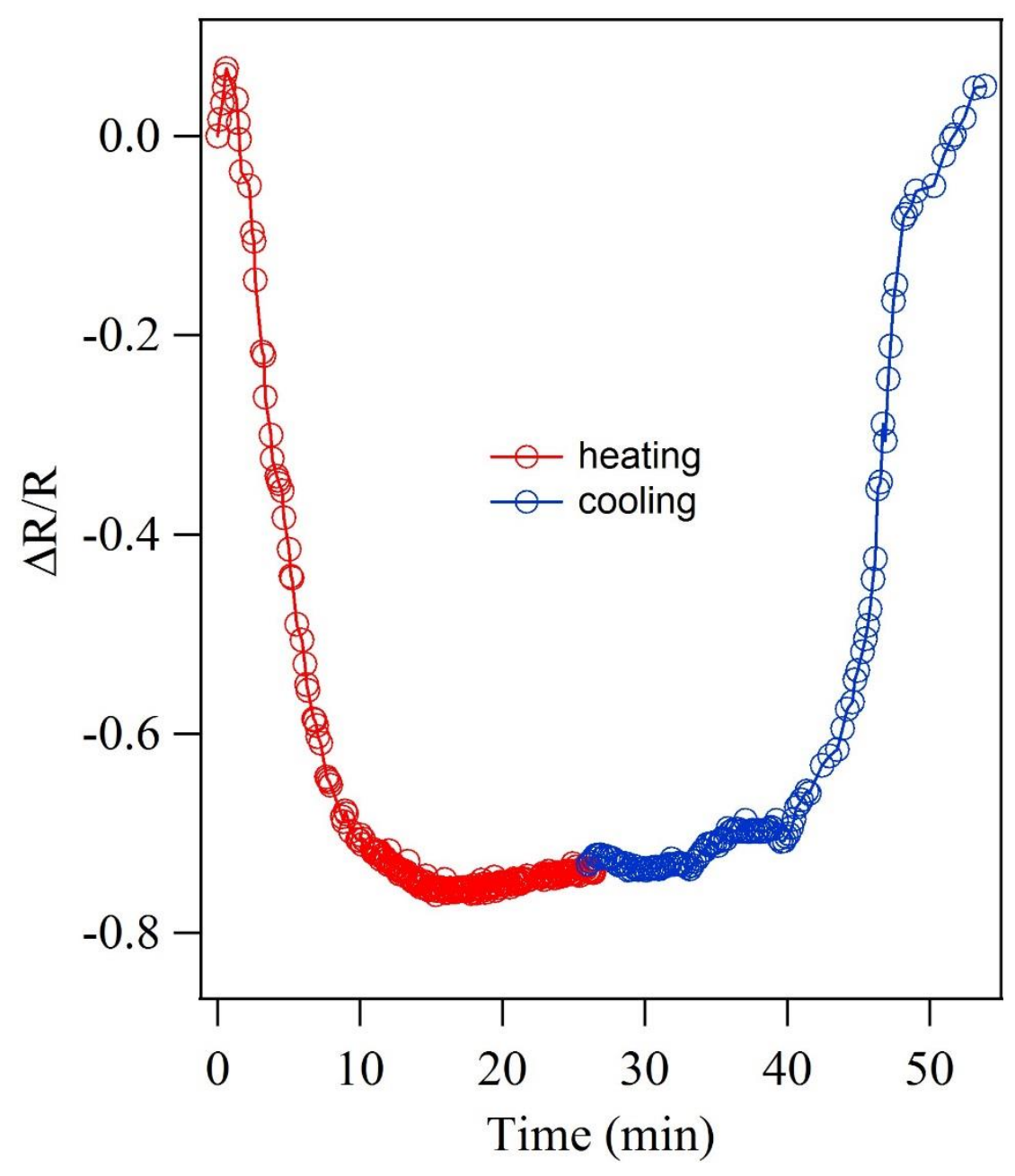

Figure 3.5: $\quad$ Relative change in the resistance of phosphorene as it is heated and cooled versus time. 


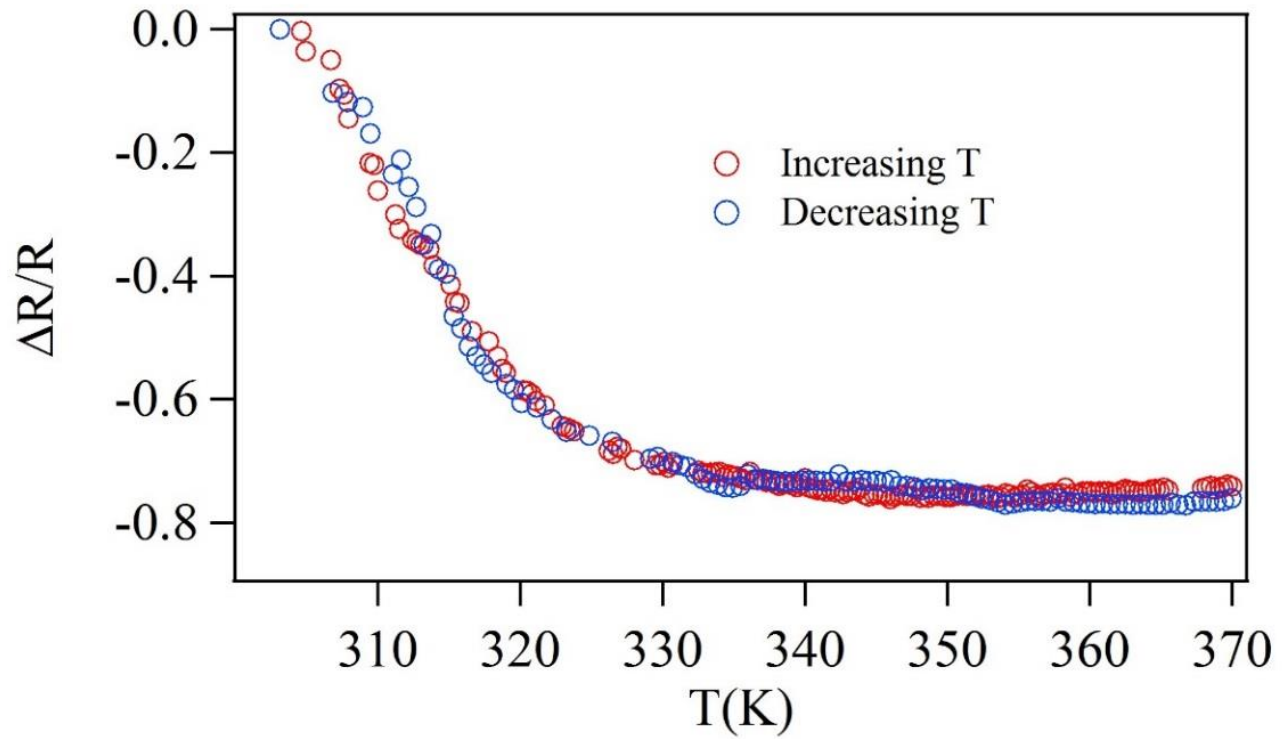

Figure 3.6

Relative changes in the resistance of phosphorene as it is heated and cooled versus temperature.

The relative changes in resistance as the sample is heated is shown in figure 3.5 and 3.6. Initial temperature was $25^{\circ} \mathrm{C}$ and max temperature was $100{ }^{\circ} \mathrm{C}$. A decrease in resistance as temperatures increase is typical for semiconductors as charge carriers are thermally excited across the energy band gap. In order to improve the noise to data ratio the conducting channel was opened $\left(\mathrm{V}_{\mathrm{ds}}=0.2 \mathrm{~V}, \mathrm{~V}_{\mathrm{g}}=-20 \mathrm{~V}\right)$ for all relative resistance measurements. There is a $75 \%$ relative decrease in resistance as the sample is heated. Temperature heating transition was done in a controlled linear manor. As such, an approximation of the energy band gap for phosphorene was determined.

Using the relationship,

$$
n \propto \exp \left(-E_{g} / 2 k_{b} T\right)
$$

with $\mathrm{n}$ being density of states in conduction band, $\mathrm{E}_{\mathrm{g}}$ is the energy band gap, $\mathrm{k}_{\mathrm{b}}$ is the Boltzmann constant, and $\mathrm{T}$ is temperature. The density of states is proportional to the 
conductivity which is inversely proportional to the resistivity and resistance. Therefore the resistance

$$
R \propto \exp \left(E_{g} / 2 k_{b} T\right)
$$

with R equal to resistance. From the current works data, energy band gap for phosphorene is approximated at $1.8 \mathrm{eV}$. This is lower than the $2.09 \mathrm{eV}$ found from photoluminescence. This difference may be due to the measured resistance including the contact resistances. The total resistance of the system is being measured and must take into account the contact resistances due to the setup being a two-probe setup. Total resistance is given by $R_{t}=R_{d}+R_{s}+R_{p}$. The variables $\mathrm{R}_{\mathrm{t}}, \mathrm{R}_{\mathrm{p}}, \mathrm{R}_{\mathrm{d}}$ and $\mathrm{R}_{\mathrm{s}}$ are the total resistance, phosphorene resistance, drain contact resistance, and source contact resistance respectively. The contacts are ohmic due to asymmetry found in I-V curve which is expected from using drop casting. This is further verified by the loss of current modulation when measurements done with laboratory lights on, which suggest photovoltaic effect. In addition, $R_{d}$ and $R_{s}$ cannot be assumed to be equal to each other. Therefore the resistance of phosphorene $\mathrm{R}_{\mathrm{p}}$ cannot be determined directly from this setup. However, changes in resistance is approximated as being due solely to changes in resistance of phosphorene $R_{p}$. This is due to the potential shielding of the contacts by phosphorene, in addition to phosphorene not having a protective covering material [87, 190] which could hinder interaction of phosphorene with gases. As such $R_{d}$ and $R_{s}$ are assumed to be constant and changes in total resistance is given by,

$$
\Delta R_{t}=R_{d}-R_{d}+R_{s}-R_{s}+R_{p f}-R_{p i}=\Delta R_{p}
$$


which shows that $R_{d}$ and $R_{S}$ can be approximated as canceling out. Initial phosphorene resistance is defined as $R_{\mathrm{pi}}$ and finial resistance is $\mathrm{R}_{\mathrm{pf}}$. Due to equation 3.04 it is better to look at the changes in resistance. The relative change in resistance can be written as,

$$
\frac{\Delta R_{T}}{\mathrm{R}_{p i}}=\frac{\Delta R_{p}}{\mathrm{R}_{p i}}
$$

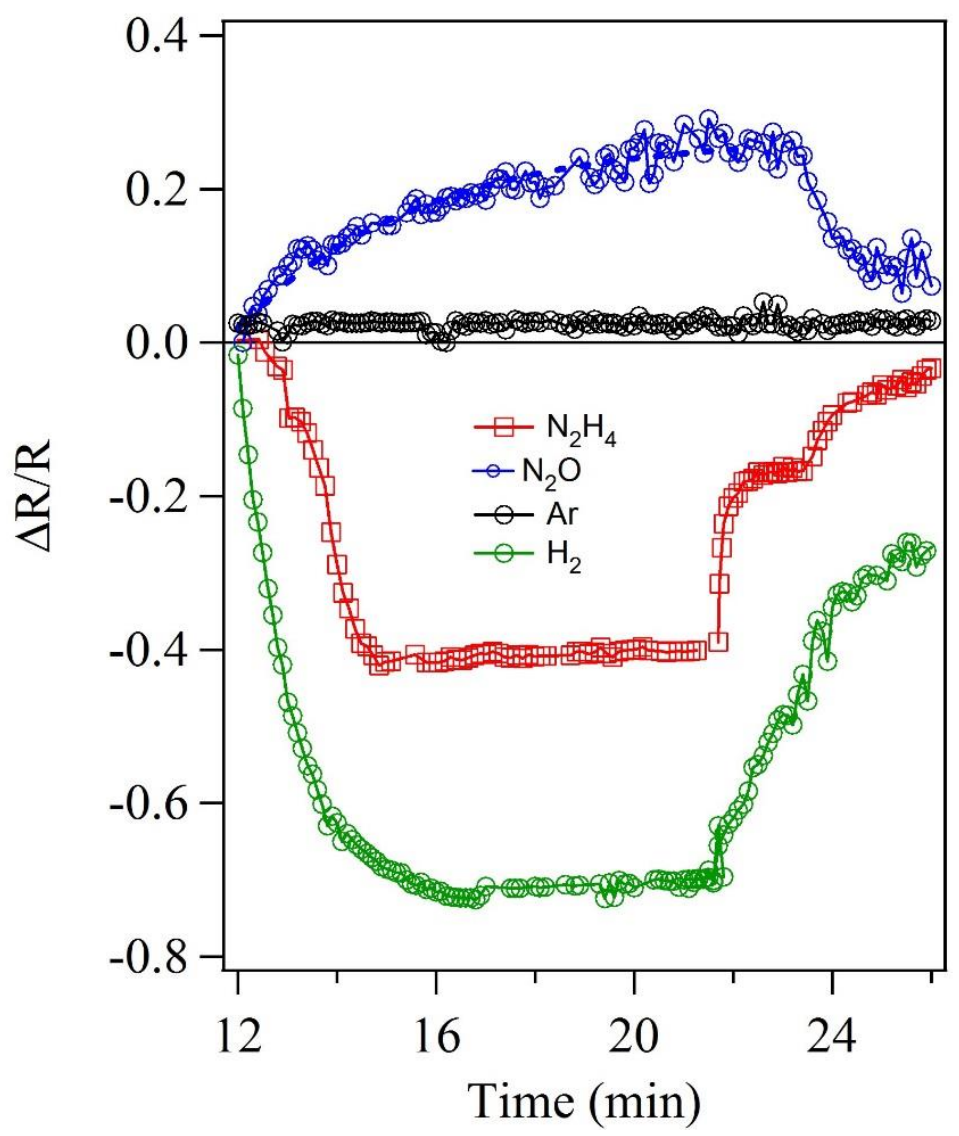

Figure 3.7:

Relative change in resistance in phosphorene as it is exposed to various gases at $60^{\circ} \mathrm{C}$. 


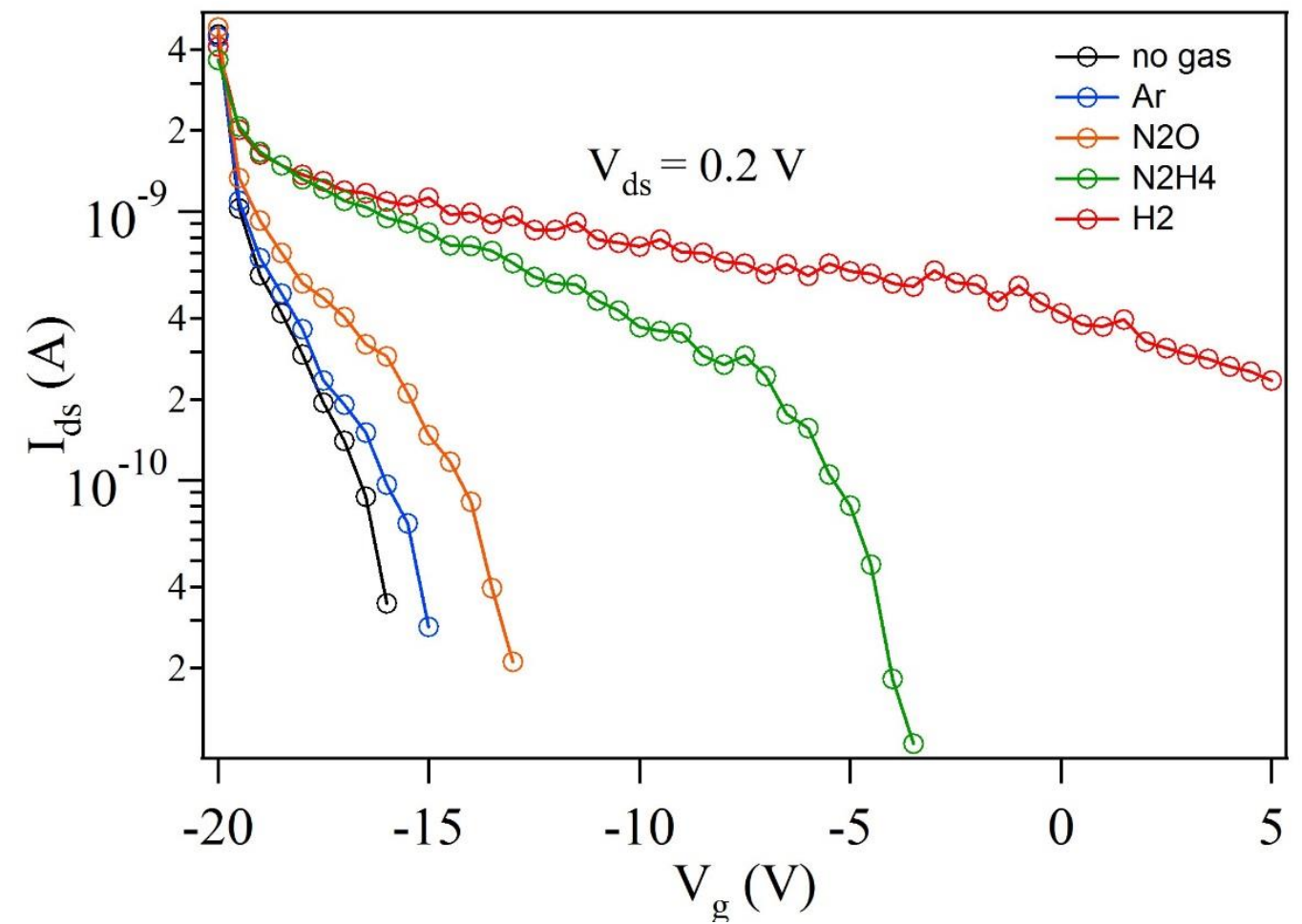

Figure 3.8: $\quad$ Transfer characteristics of phosphorene as it is exposed to various gases at $60^{\circ} \mathrm{C}$

Figure 3.8 shows relative resistance changes as phosphorene is exposed to different gases at $60^{\circ} \mathrm{C}$. Transfer characteristics of phosphorene in different gases $\left(\mathrm{V}_{\mathrm{ds}}=\right.$ $0.2 \mathrm{~V}$ ) is shown in figure 3.9. With no gas and pressure below 10 Torr it is shown that pinch off (flat-band condition) occurs at about $\mathrm{V}_{\mathrm{g}}=-16.5 \mathrm{~V}$. This rapid modulation of current is in contrast to previous works that reach pinch off at much higher gate voltage values [86-88, 202]. Decreasing current values as gate voltage becomes increasingly positive indicates that the phosphorene FET is p-type. In the hole doping region the mobility was calculated using the conventional equation for transconductance $\mu_{h}=$ $\left(d I_{d s} / d V_{g}\right)(L / W)\left[\left(C_{o x} * V_{g}\right)^{-1}\right]$ defined in section 3.1. The gate oxide thickness is $\mathrm{d}_{\mathrm{ox}}=$ $300 \mathrm{~nm}$ and $\varepsilon_{\mathrm{ox}}=3.5 * 10^{-11} \mathrm{~F} / \mathrm{m}$ is the $\mathrm{SiO}_{2}$ dielectric constant. Experimentally determined hole mobility was about $26 \mathrm{~cm}^{2} / \mathrm{Vs}$. This is higher than $14 \mathrm{~cm}^{2} / \mathrm{Vs}$ found for 
pulsed laser deposited thin film black phosphorus [202], but lower than the $100 \mathrm{~cm}^{2} / \mathrm{Vs}$ found for mechanical exfoliated phosphorene flakes [88]. During gas exposure, the conducting channel of the FET (phosphorene) was opened by setting $V_{g}=-20 \mathrm{~V}$ and $V_{d s}$ $=0.2 \mathrm{~V}$ for resistance measurements. Argon exposure produces a slight increase in the resistance of phosphorene. The relative change is below 10\%. Transfer characteristics also show a slight increase in voltages compared to non-exposed phosphorene. Channel pinch off happens at a higher voltage also. These small changes in transport properties show that argon environments may be useful in protecting phosphorene without significantly changing its properties.

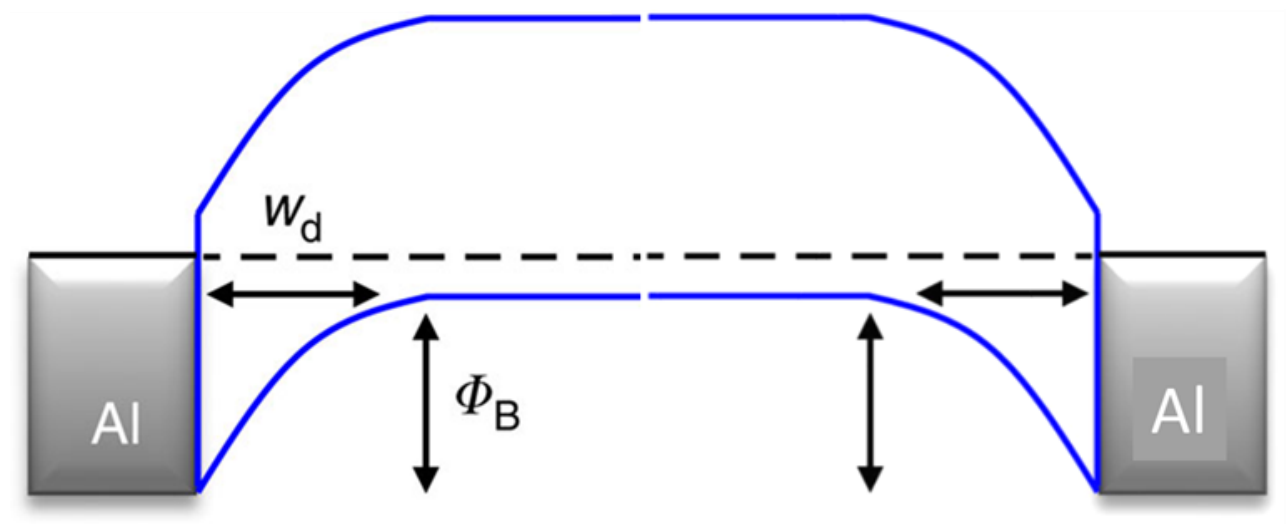

Figure 3.9: Al contacts.

Schematic of band diagram showing how the schottky barrier is formed between phosphorene and

Changes in phosphorene when exposed to hydrogen is more pronounced. The hydrogen/phosphorene interaction has been calculated to be a physisorbed process that is reversible [203]. Hydrogen is a highly reducing gas. Reducing gases are expected to give electrons and for a p-type semiconductor this should result in increased resistance and a decrease in current as holes are depleted. That is true for a charge transfer process during 
physical absorption. However, figure 3.8 shows a large relative decrease in resistance which shows that hydrogen is not acting as a reducing agent. The reason for the decrease in resistance may be due to hydrogen atoms interacting with the phosphorene/Al interface. Phosphorene was drop cast on top of the contacts, which forms a schottky barrier. Hydrogen is decreasing the schottky barrier, which allows holes to transfer easier. More holes decreases resistance and increases current values in the hole conduction region. The above statement is depicted in figure 3.9. There is also a complete loss of current modulation in the transfer characteristics (figure 3.8) caused by more holes and an increase in current. The relative decrease in resistance is about $70 \%$. This is an extremely large value. The $70 \%$ decrease due to hydrogen is actually compounded on top of the $75 \%$ heat related change. This shows potential applications of phosphorene as a material for hydrogen storage and release. The above may be possible with a calculated binding energy of $0.13 \mathrm{eV} / \mathrm{H}_{2}$ [203] for the hydrogen/phosphorene system. Release of hydrogen can be inferred from the increase in resistance as hydrogen is removed in figure 3.7. Due to phosphorene not returning to its initial value, it may be inferred that hydrogen was not fully released. Phosphorene reaches $30 \%$ of its original resistance which is directly related to percentage of unreleased hydrogen. Exposure of phosphorene to nitrous oxide shows an increase in resistance (about 27\%) and only a slight change in transfer characteristics. Nitrous oxide is a strong oxidizing gas. Nitrous oxide is increasing the schottky barrier making hole conduction more difficult. The increased schottky barrier, causes a decrease in holes which in turn increases resistance in phosphorene. Once $\mathrm{N}_{2} \mathrm{O}$ is removed, relative resistance values approach initial values. The above shows that $\mathrm{N}_{2} \mathrm{O}$ exposure effects are more easily reversed. Analysis was also done on highly reactive 
hydrazine. Figure 3.7 shows that the relative decrease in resistance is about $40 \%$, which is not as large a change as exposure to hydrogen. The transfer curve also shows current modulation is not entirely loss, however it is shifted to much higher gate voltages compare to unexposed phosphorene. Hydrazine acts similar to hydrogen on phosphorene. The effect is not as pronounced though, which may be due to the increased size of the $\mathrm{N}_{2} \mathrm{H}_{4}$ molecule. Release of hydrazine appears to be easier in comparison to the release of hydrogen as the resistance approaches initial values.

\subsubsection{Light Interaction}

LED's used in this experiment are connected to separate contacts away from the substrate. However, the LED itself is positioned to ensure the majority of its light is directed towards the sample. As an added precaution, laboratory lights were cut off during measurements. The analysis chamber was evacuated to prevent sample degradation and shielded with light absorbing black-out material.

Transfer curves are shown in figure 3.10. For no light exposure, the phosphorene FET shows characteristic current modulation, and p-type conductivity. However, for every color LED used, all current modulation is lost in the FET once the LED is turned on. The above finding is interesting as it is only seen when phosphorene is exposed to light. This loss of current modulation is even seen by exposing the sample to common laboratory room lights in the ceiling. In photo-detecting systems, this property can be exploited to create novel 2D light sensors. However, for FET use in logic constructs this 
feature may be an undesirable affect. All measurements done in this section were done at room temperature.

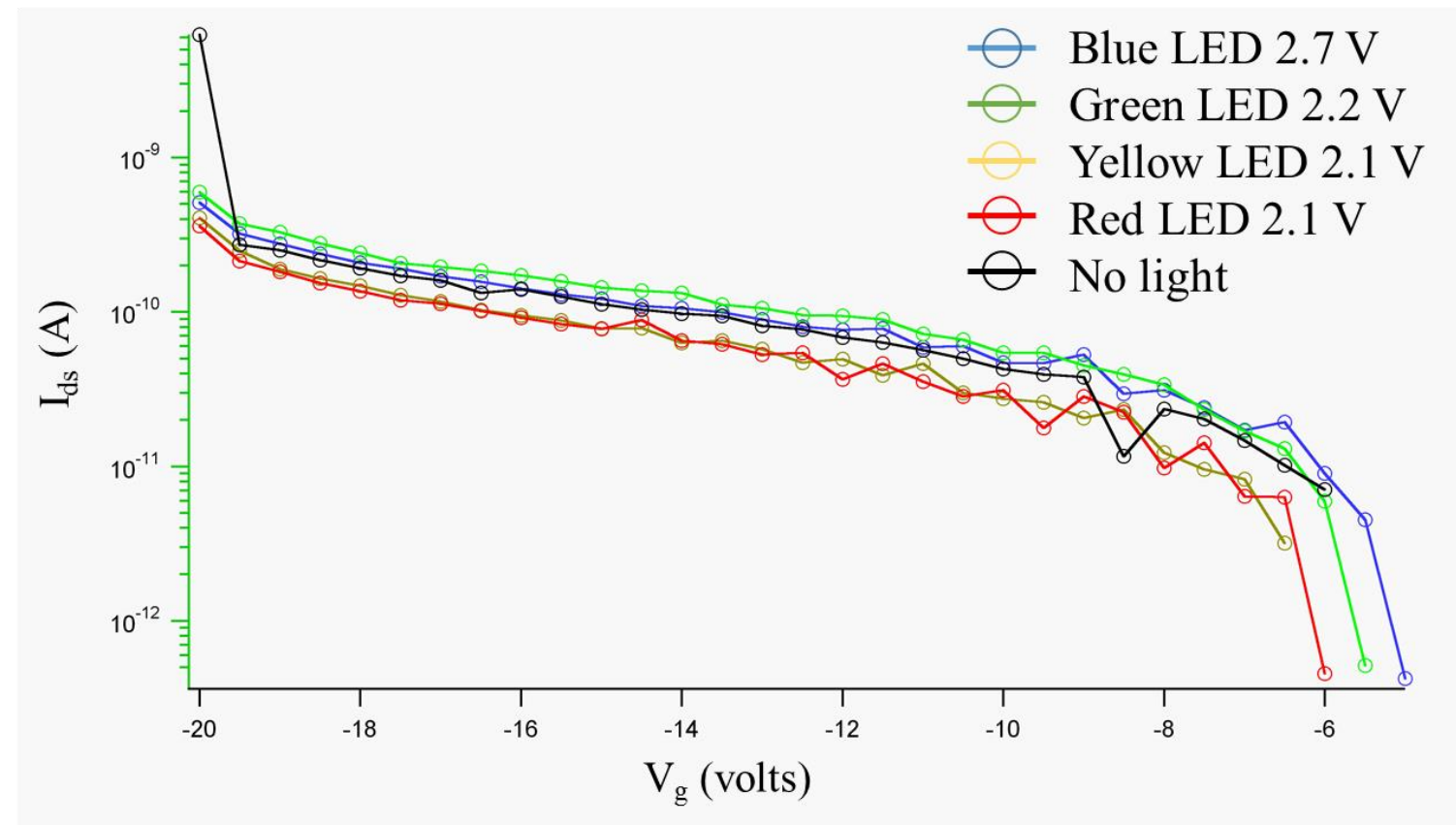

Figure 3.10: $\quad$ Transfer characteristics of phosphorene as it is exposed to various LED's

\begin{tabular}{ll} 
Light Conditions & Mobility $\left(\mathrm{cm}^{2} / \mathrm{Vs}\right)$ \\
\hline No light & 25.60 \\
Red LED & 0.317 \\
Yellow LED & 0.346 \\
Green LED & 0.493 \\
Blue LED & 0.413 \\
\hline
\end{tabular}

Figure 3.11: $\quad$ Hole mobility in phosphorene for exposure to different LED's 
The mobility of the holes in phosphorene was calculated using the transconductance equation $\mu_{h}=\left(d I_{d s} / d V_{g}\right)(L / W)\left[\left(C_{o x} * V_{d s}\right)^{-1}\right]$. The variables have been defined in section 3.1. For hole mobility, the calculation was done in the hole conduction region. $\mathrm{C}_{\mathrm{ox}}$ is stated once more as the capacitance of the gate oxide and defined as $\mathrm{C}_{\mathrm{ox}}=\varepsilon_{\mathrm{ox}} / \mathrm{d}_{\mathrm{ox}}[87,88]$. The gate oxide thickness is $\mathrm{d}_{\mathrm{ox}}=300 \mathrm{~nm}$ and $\varepsilon_{\mathrm{ox}}=3.5 *$ $10^{-11} \mathrm{~F} / \mathrm{m}$ is the $\mathrm{SiO}_{2}$ dielectric constant. $\mathrm{V}_{\mathrm{ds}}$ is the drain-source voltage and has a value of $0.2 \mathrm{~V}$. The hole mobility drastically decreases when exposed to light. All values drop below $1 \mathrm{~cm}^{2} / \mathrm{Vs}$. There is not much difference between the values of mobility when comparing the different LED's. Figure 3.11 shows mobility under different conditions.

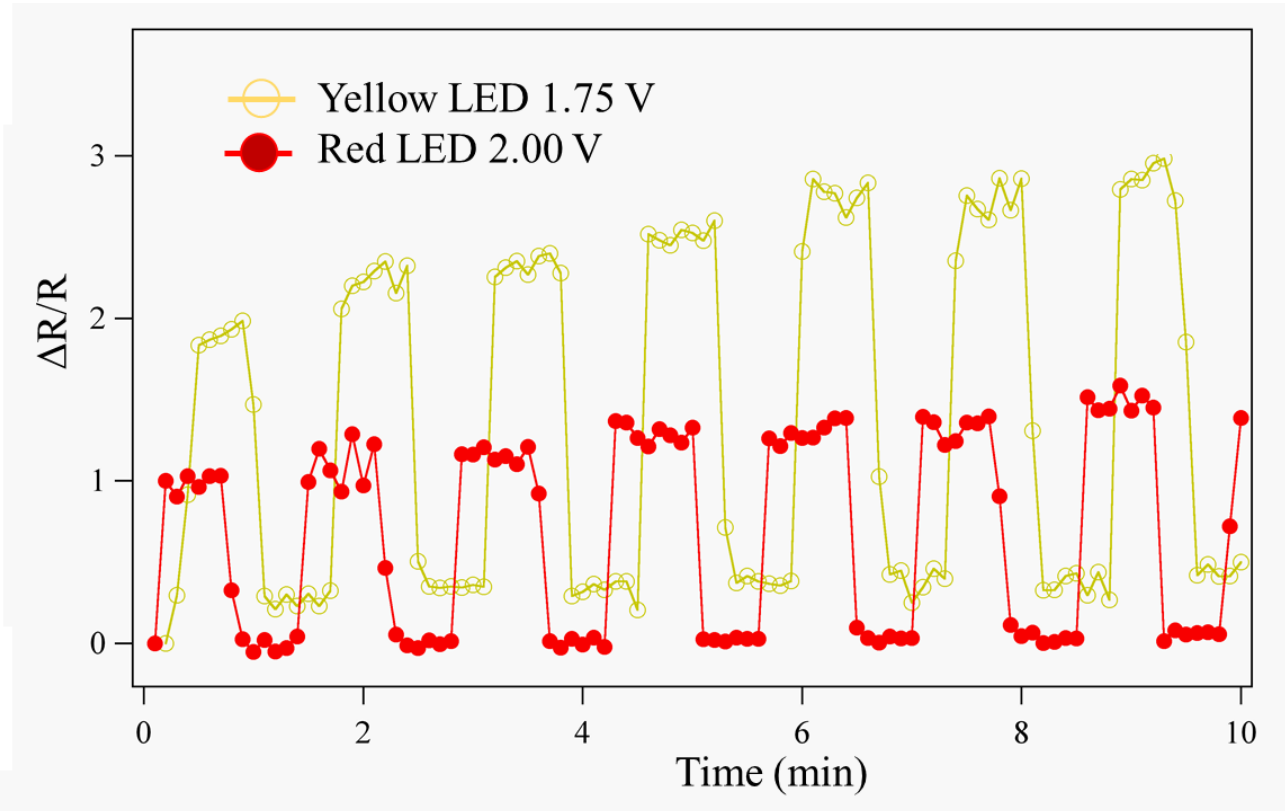

Figure $3.12 \quad$ Relative resistance of phosphorene exposed to red and yellow LEDs.

Combining the decrease in hole mobility with the loss of current modulation due to light, is a signal that the light sources are generating charge carriers in phosphorene. As more charge carriers are excited, current modulation is no longer possible. Also short 
lived excited electrons may increase recombination of holes, which is evident by decreased hole mobility. Figure 3.13 shows how the electrons are excited by the LED light. Red light having an energy of $1.65 \mathrm{eV}$ to $2.00 \mathrm{eV}$ is about the same energy as the band gap of phosphorene (about $2.09 \mathrm{eV}$ ). Yellow light having an energy $2.10 \mathrm{eV}$ to 2.17 $\mathrm{eV}$ is slightly larger. Both red and yellow light appear to be able to excite electrons, however the larger energy of the yellow LED may be why it increases phosphorene's resistance more (figure 3.12).

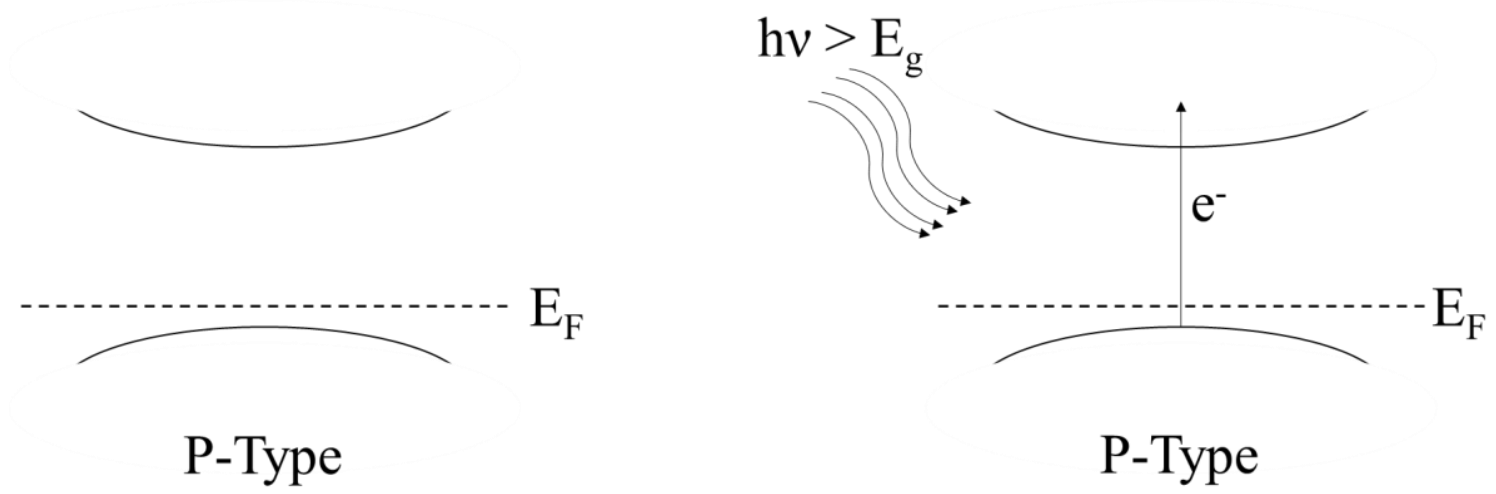

Figure 3.13: $\quad$ Schematic of band diagram of a.) pristine phosphorene b.) and phosphorene exposed to light with energy greater than phosphorene's band gap.

Figure 3.12, shows the relative resistance of phosphorene as it is exposed to light. The resistance increases for constant $\mathrm{V}_{\mathrm{ds}}$ when the LED's are tuned on. It should be noted that the duration of light exposure was the same for all LED's during the relative resistance versus time measurements but they may be shifted with respect to one another due to different starting times. For red LED exposure, the increase in resistance is one whole factor in comparison to the original resistance. The yellow LED has a similar effect that is over twice as large. It is noted that during resistance measurements the change in resistance is not measured if the phosphorene conducting channel is not opened 
by applying a $\mathrm{V}_{\mathrm{g}}$ less than $-10 \mathrm{~V}$. It is seen that the increase in resistance for each exposure has some variation. This variation may be due to non-consistent output from the LED's.
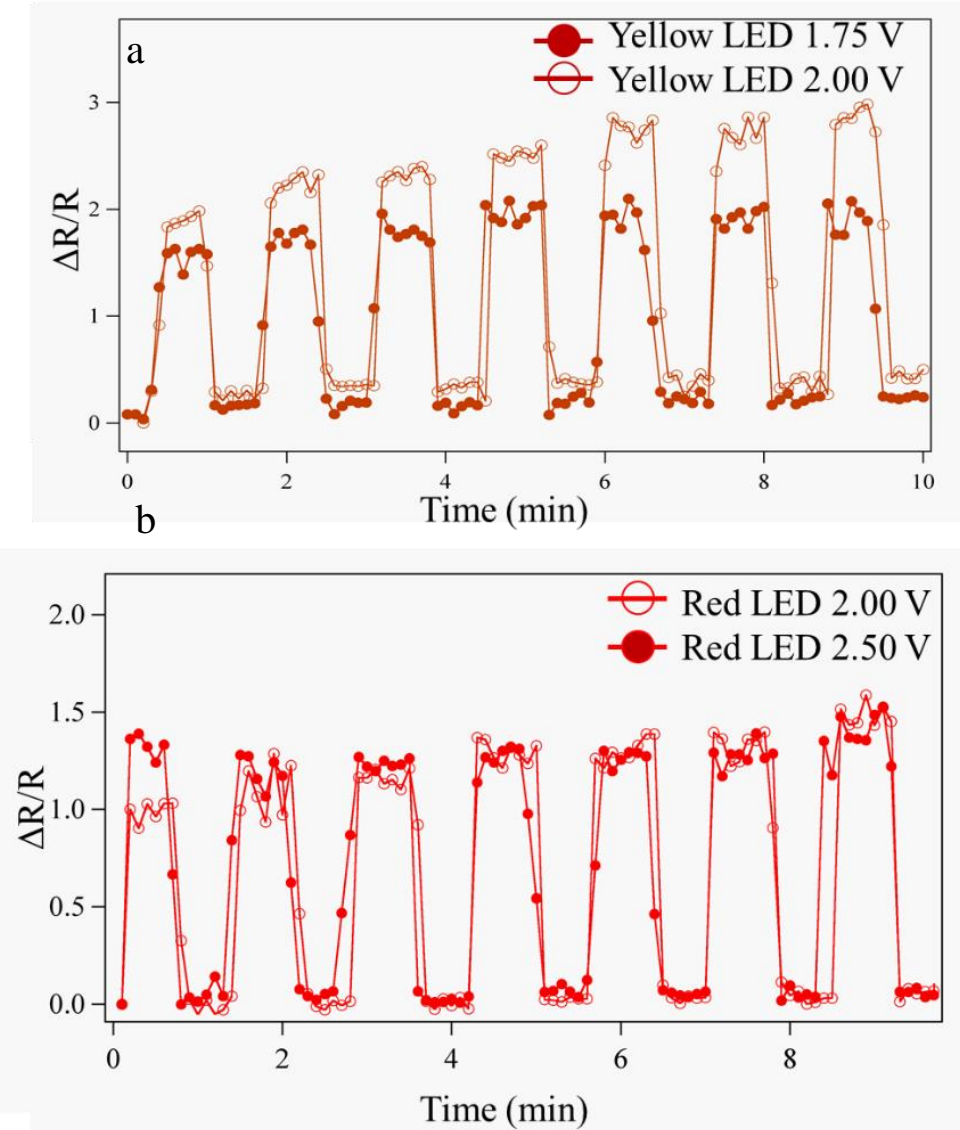

Figure 3.14: $\quad$ Relative changes in resistance for phosphorene exposed to a.) yellow led and b.) red led for different led voltages

Also figure 3.14, shows that the driving voltage of the LED's has little effect on changes in phosphorene. These finding show that light can drastically alter the properties of phosphorene based devices by decreasing hole mobility. 


\section{CHAPTER 4 - CONCLUSIONS}

Technology is constantly being pushed further and further. As electronics are being scaled at rapid paces, limits are becoming evident. 2D materials may be a viable route for continued decrease in size and increase of performance for electronic devices. With the advent of Graphene, the possibilities of 2D materials became evident. As more and more materials are discovered each day, more research is needed to understand the capabilities of these novel systems.

In order to advance the properties of semiconductors, heterostructures can be employed. For 2D systems this is vital to tailoring their properties and developing novel devices. Stacked structures of transition metal dichalcogenides have been thoroughly studied due their ease of synthesis. However, making in-plane heterostructures has proven to be much more difficult. In-plane heterostructures due to their chemical bonding afford new properties. As such, the current work has developed a novel method to grow both in-plane and stacked structures in the same system. Initially using thermal evaporation of transition metal oxides allowed for precise patterns and layer control to be synthesized. Following thermal evaporation, chemical vapor deposition was used to convert the oxide species to dichalcogenide species. For systems that underwent only one chemical vapor deposition (Series A), raman spectroscopy showed that these samples are highly alloyed. The transition metal atoms from each layer undergoes an interlayer 
exchange causing a mixed system to develop. Molybdenum atoms occupy sites that should have tungsten atoms and vice versa. This is proved by the appearance of the alloy peak identified in raman. The exchanging of atoms is believed to be due to the lower stability of the oxides, which increases their surface diffusion across the substrate surface. Even with this increased atomic movement, raman mapping shows that the patterns formed by thermal evaporation are not lost through the chemical vapor deposition process. In fact the transition metal has very little lateral movement even though it has interlayer exchange.

For transition metal dichalcogenides grown using two chemical vapor depositions (Series B), less interlayer transition metal exchange is found. The raman peaks have much smaller full with at half max, which is an indicator of improved crystalline structure. In addition, there is no alloy related peaks found in the heterostructure system. Series B method allows for low atomic movement in both the in-plane and vertical directions. One of the key aspects of this method is the ability to grow samples with the same transition metal but different chalcogen atoms in different layers. This was not possible with series A since it only required one chalcogenization step. By utilizing this aspects of series B, the bilayer heterostructure system of $\mathrm{WSe}_{2} / \mathrm{WS}_{2}$ was able to be successfully synthesized. This particular system is of great importance due to predictions that it may have a direct band gap. It is known from most transition metal dichalcogenide 2D systems that only monolayers have direct band gaps. Having multiple layers with a direct band gaps is important for improving optoelectronic properties.

The amazing properties of phosphorene has also been studied. Phosphorene has many properties, such as topological induced anisotropy and high hole mobility. Unlike 
graphene, phosphorene is not atomically flat due to $\mathrm{sp}^{3}$ bonding. This same bonding leaves each phosphorus atom with a lone pair of electrons. All of these properties of phosphorene gives scientists and engineers many options for its use in optoelectronic devices. However, these same properties can alter device performance.

Phosphorene was synthesized in the current work using liquid mechanical exfoliation of bulk black phosphorus. Liquid mechanical exfoliation was able to produce monolayer phosphorene. Compared to previous research, the current works exfoliation of phosphorene was done in less time. Scanning electron microscopy showed several phosphorene flakes that were overlapping. Photoluminescence also showed several peaks due to non-uniformity. In addition, photoluminescence showed a strong peak at $2.09 \mathrm{eV}$ which is attributed to monolayer phosphorene. Transmission electron microscopy showed that phosphorene forms folds similar to graphene.

Field effect transistors were fabricated in the current work, using phosphorene as the conducting channel. The device was found to have p-type behavior. Previous works found that phosphorene has a direct band gap. Experimentally, the band gap has been found to be in the visible region for the current work. Visible light band gaps allow for charge carriers to be easily excited optically. The current work has found that light absorption can drastically alter the properties of a field effect transistor based on phosphorene. Using various colored light emitting diodes, an in-situ analysis of the sample was done. It was found that current modulation was lost in the field effect transistor every time that it was irradiated with light. Current modulation loss was independent of color light emitting diode used. The above finding shows that care must be taken if phosphorene based field effect transistors are used in logic circuits. Any 
visible light can cause the transistor conducting channel to be open. On the other hand, this setup may be exploited in a photodetector system. By analyzing the relative resistance of phosphorene as it is exposed to light, it was found that its resistance increased in a consistent manor. Novel 2D photodetectors may be a viable use of phosphorene's abilities.

It was also vital to evaluate the field effect transistor performance in various gas environments. Phosphorene's inplane bonding leaves lone electron pairs of each phosphorus atom which reacts with other atoms. By using a controlled environment, exposure of phosphorene to different gases caused major changes to its transport properties. It was found that hydrogen lowers the schottky barrier by interacting with the phosphorene/Al interface. The relative resistance decreases as a result. The current work has also shown that the partial release of hydrogen can be done at $60{ }^{\circ} \mathrm{C}$ temperature. Hydrazine had a similar effect, however phosphorene more readily released the hydrazine molecules. This release may be impart caused by the increased size of the hydrazine molecule compared to hydrogen. Nitrous Oxide had the opposite effect. Relative resistance increased in phosphorene when exposed to nitrous oxide. Due to the inert nature of argon (caused by its closed outer electron shell), it had very little effect on phosphorene which shows it to be suitable for safe storage of phosphorene based devices. The above shows that novel devices fabricated from phosphorene must be studied carefully to fully understand how they will behave in the variable environments that they may meet in real world conditions. 


\section{REFERENCES}

1. Berkdemir, A., et al., Identification of individual and few layers of WS2 using Raman Spectroscopy. Scientific Reports, 2013. 3: p. Article Number: 1755

2. Ding, Y., et al., First principles study of structural, vibrational and electronic properties of graphene-like $M X(2)(M=M o, N b, W, T a ; X=S$, Se, Te) monolayers. Physica BCondensed Matter, 2011. 406(11): p. 2254-2260.

3. Kang, J., et al., Band offsets and heterostructures of two-dimensional semiconductors. Applied Physics Letters, 2013. 102(1): p. Article Number: 012111.

4. Ataca, C., H. Sahin, and S. Ciraci, Stable, Single-Layer MX2 Transition-Metal Oxides and Dichalcogenides in a Honeycomb-Like Structure. Journal of Physical Chemistry C, 2012. 116(16): p. 8983-8999.

5. Waghray, H., T.S. Lee, and B.J. Tatarchuk, A study of the tribological and electrical properties of sputtered and burnished transition metal dichalcogenide films. Surface \& Coatings Technology, 1995. 76-77(1-3): p. 415-420.

6. Rapoport, L., et al., Mechanism of friction of fullerenes. Industrial Lubrication and Tribology, 2002. 54(4): p. 171-176.

7. Novoselov, K.S., et al., Electric field effect in atomically thin carbon films. Science, 2004. 306(5696): p. 666-669.

8. Merki, D. and X.L. Hu, Recent developments of molybdenum and tungsten sulfides as hydrogen evolution catalysts. Energy \& Environmental Science, 2011. 4(10): p. 38783888.

9. Kibsgaard, J., et al., Engineering the surface structure of MoS2 to preferentially expose active edge sites for electrocatalysis. Nature Materials, 2012. 11(11): p. 963-969.

10. Hinnemann, B., et al., Biornimetic hydrogen evolution: MoS2 nanoparticles as catalyst for hydrogen evolution. Journal of the American Chemical Society, 2005. 127(15): p. 5308-5309.

11. Jaramillo, T.F., et al., Identification of active edge sites for electrochemical $\mathrm{H}-2$ evolution from MoS2 nanocatalysts. Science, 2007. 317(5834): p. 100-102.

12. Raybaud, P., et al., Ab initio study of the H-2-H2S/MoS2 gas-solid interface: The nature of the catalytically active sites. Journal of Catalysis, 2000. 189(1): p. 129-146.

13. Gutierrez, H.R., et al., Extraordinary Room-Temperature Photoluminescence in Triangular WS2 Monolayers. Nano letters, 2013. 13(8): p. 3447-3454.

14. Tonndorf, P., et al., Photoluminescence emission and Raman response of monolayer MoS2, MoSe2, and WSe2. Optics Express, 2013. 21(4): p. 4908-4916.

15. Sahin, H., et al., Anomalous Raman spectra and thickness-dependent electronic properties of WSe2. Physical Review B, 2013. 87(16).

16. Yamamoto, M., et al., Strong Enhancement of Raman Scattering from a Bulk-Inactive Vibrational Mode in Few-Layer MoTe2. ACS Nano, 2014. 8(4): p. 3895-3903.

17. Horzum, S., et al., Phonon softening and direct to indirect band gap crossover in strained single-layer MoSe\$\{\}_\{2\}\$. Physical Review B, 2013. 87(12): p. 125415.

18. Wang, Q.H., et al., Electronics and optoelectronics of two-dimensional transition metal dichalcogenides. Nature Nanotechnology, 2012. 7(11): p. 699-712.

19. Mak, K.F., et al., Atomically Thin MoS2: A New Direct-Gap Semiconductor. Physical Review Letters, 2010. 105(13). 
20. Lee, C., et al., Anomalous Lattice Vibrations of Single- and Few-Layer MoS2. Acs Nano, 2010. 4(5): p. 2695-2700.

21. Splendiani, A., et al., Emerging Photoluminescence in Monolayer MoS2. Nano Letters, 2010. 10(4): p. 1271-1275.

22. Zhang, W., et al., High-Gain Phototransistors Based on a CVD MoS2 Monolayer. Advanced Materials, 2013. 25(25): p. 3456-3461.

23. Radisavljevic, B. and A. Kis, Mobility engineering and a metal-insulator transition in monolayer MoS2. Nat Mater, 2013. 12(9): p. 815-820.

24. Li, H., et al., From Bulk to Monolayer MoS2: Evolution of Raman Scattering. Advanced Functional Materials, 2012. 22(7): p. 1385-1390.

25. Eda, G., et al., Photoluminescence from Chemically Exfoliated MoS(2). Nano Letters, 2011. 11(12): p. 5111-5116.

26. Lee, H.S., et al., MoS2 Nanosheet Phototransistors with Thickness-Modulated Optical Energy Gap. Nano Letters, 2012. 12(7): p. 3695-3700.

27. Najmaei, S., et al., Thermal effects on the characteristic Raman spectrum of molybdenum disulfide (MoS(2)) of varying thicknesses. Applied Physics Letters, 2012. 100(1): p. 013106.

28. Sahoo, S., et al., Temperature-Dependent Raman Studies and Thermal Conductivity of Few-Layer MoS2. Journal of Physical Chemistry C, 2013. 117(17): p. 9042-9047.

29. Mak, K.F., et al., Atomically Thin MoS(2): A New Direct-Gap Semiconductor. Physical Review Letters, 2010. 105(13): p. Article Number: 136805.

30. Splendiani, A., et al., Emerging Photoluminescence in Monolayer MoS(2). Nano Letters, 2010. 10(4): p. 1271-1275.

31. Lee, C., et al., Anomalous Lattice Vibrations of Single- and Few-Layer MoS(2). Acs Nano, 2010. 4(5): p. 2695-2700.

32. Molina-Sanchez, A. and L. Wirtz, Phonons in single-layer and few-layer MoS2 and WS2. Physical Review B, 2011. 84(15): p. 155413.

33. Bertolazzi, S., J. Brivio, and A. Kis, Stretching and Breaking of Ultrathin MoS2. ACS Nano, 2011. 5(12): p. 9703-9709.

34. Björkman, T., et al., van der Waals Bonding in Layered Compounds from Advanced Density-Functional First-Principles Calculations. Physical Review Letters, 2012.

108(23): p. 235502.

35. Bernardi, M., M. Palummo, and J.C. Grossman, Extraordinary Sunlight Absorption and One Nanometer Thick Photovoltaics Using Two-Dimensional Monolayer Materials. Nano Letters, 2013. 13(8): p. 3664-3670.

36. Najmaei, S., et al., Vapour phase growth and grain boundary structure of molybdenum disulphide atomic layers. Nature Materials, 2013. 12(8): p. 754-759.

37. Chhowalla, M., et al., The chemistry of two-dimensional layered transition metal dichalcogenide nanosheets. Nature Chemistry, 2013. 5(4): p. 263-275.

38. Matte, H.S.S.R., et al., MoS(2) and WS(2) Analogues of Graphene. Angewandte ChemieInternational Edition, 2010. 49(24): p. 4059-4062.

39. Gong, Y., et al., Vertical and in-plane heterostructures from WS2/MoS2 monolayers. Nat Mater, 2014. 13(12): p. 1135-1142.

40. Novoselov, K.S. and A.H.C. Neto, Two-dimensional crystals-based heterostructures: materials with tailored properties. Physica Scripta, 2012. T146.

41. Geim, A.K. and I.V. Grigorieva, Van der Waals heterostructures. Nature, 2013. 499(7459): p. 419-425.

42. Kang, J., et al., Band offsets and heterostructures of two-dimensional semiconductors. Applied Physics Letters, 2013. 102(1).

43. $\quad \mathrm{Yu}, \mathrm{W} . \mathrm{J} .$, et al., Vertically stacked multi-heterostructures of layered materials for logic transistors and complementary inverters. Nature Materials, 2013. 12(3): p. 246-252. 
44. Cheng, R., et al., Electroluminescence and Photocurrent Generation from Atomically Sharp WSe2/MoS2 Heterojunction p-n Diodes. Nano Letters, 2014. 14(10): p. 5590-5597.

45. Deng, Y., et al., Black Phosphorus-Monolayer MoS2 van der Waals Heterojunction p-n Diode. Acs Nano, 2014. 8(8): p. 8292-8299.

46. Fang, H., et al., Strong interlayer coupling in van der Waals heterostructures built from single-layer chalcogenides. Proceedings of the National Academy of Sciences of the United States of America, 2014. 111(17): p. 6198-6202.

47. Tongay, S., et al., Tuning Interlayer Coupling in Large-Area Heterostructures with CVDGrown MoS2 and WS2 Monolayers. Nano Letters, 2014. 14(6): p. 3185-3190.

48. Cao, Y., et al., Quality Heterostructures from Two-Dimensional Crystals Unstable in Air by Their Assembly in Inert Atmosphere. Nano Letters, 2015. 15(8): p. 4914-4921.

49. Cui, X., et al., Multi-terminal transport measurements of MoS2 using a van der Waals heterostructure device platform. Nature Nanotechnology, 2015. 10(6): p. 534-540.

50. Huang, L., et al., Strain induced piezoelectric effect in black phosphorus and MoS2 van der Waals heterostructure. Scientific Reports, 2015. 5.

51. Li, D., et al., Nonvolatile Floating-Gate Memories Based on Stacked Black PhosphorusBoron Nitride-MoS2 Heterostructures. Advanced Functional Materials, 2015. 25(47): p. 7360-7365.

52. Yuan, J., et al., Photoluminescence Quenching and Charge Transfer in Artificial Heterostacks of Monolayer Transition Metal Dichalcogenides and Few-Layer Black

Phosphorus. Acs Nano, 2015. 9(1): p. 555-563.

53. Zhang, Z.Y., et al., Bandgap engineering in van der Waals heterostructures of blue phosphorene and MoS2: A first principles calculation. Journal of Solid State Chemistry, 2015. 231: p. 64-69.

54. Yin, Z.Y., et al., Single-Layer MoS2 Phototransistors. ACS Nano, 2012. 6(1): p. 74-80.

55. Britnell, L., et al., Strong Light-Matter Interactions in Heterostructures of Atomically Thin Films. Science, 2013. 340: p. 1311-1314.

56. Lee, H.S., et al., MoS2 Nanosheets for Top-Gate Nonvolatile Memory Transistor Channel. Small, 2012. 8(20): p. 3111-3115.

57. Jariwala, D., et al., Band-like transport in high mobility unencapsulated single-layer MoS2 transistors. Applied Physics Letters, 2013. 102(17): p. Article Number: 173107

58. Late, D.J., et al., Hysteresis in Single-Layer MoS2 Field Effect Transistors. Acs Nano, 2012. 6(6): p. 5635-5641.

59. Radisavljevic, B., et al., Single-layer MoS(2) transistors. Nature Nanotechnology, 2011. 6(3): p. 147-150.

60. Pospischil, A., M.M. Furchi, and T. Mueller, Solar-energy conversion and light emission in an atomic monolayer $p$-n diode. Nature Nanotechnology, 2014. 9(4): p. 257-261.

61. Haigh, S.J., et al., Cross-sectional imaging of individual layers and buried interfaces of graphene-based heterostructures and superlattices. Nature Materials, 2012. 11(9): p. 764-767.

62. Liu, Z., et al., Direct Growth of Graphene/Hexagonal Boron Nitride Stacked Layers. Nano Letters, 2011. 11(5): p. 2032-2037.

63. Terrones, H., F. Lopez-Urias, and M. Terrones, Novel hetero-layered materials with tunable direct band gaps by sandwiching different metal disulfides and diselenides.

Scientific Reports, 2013. 3: p. Article number:1549, doi:10.1038/srep01549.

64. Ming-Hui Chiu, et al., Determination of band alignment in transition metal dichalcogenides heterojunctions. arXiv:1406.5137 [cond-mat.mtrl-sci], 2014.

65. Marco M. Furchi, et al., Photovoltaic effect in an electrically tunable van der Waals heterojunction. arXiv:1403.2652 [cond-mat.mes-hall], 2014. 
66. Rui Cheng, et al., Electroluminescence and photocurrent generation from atomically sharp WSe2/MoS2 heterojunction p-n diodes. arXiv:1403.3447 [cond-mat.mes-hall], 2014.

67. Bridgman, P.W., TWO NEW MODIFICATIONS OF PHOSPHORUS. Journal of the American Chemical Society, 1914. 36(7): p. 1344-1363.

68. Karttunen, A.J., M. Linnolahti, and T.A. Pakkanen, Structural Principles of Polyhedral Allotropes of Phosphorus. ChemPhysChem, 2008. 9(17): p. 2550-2558.

69. Chou, T.D., et al., The management of white phosphorus burns. Burns, 2001. 27(5): p. 492-497.

70. Clark, S.M. and J.M. Zaug, Compressibility of cubic white, orthorhombic black, rhombohedral black, and simple cubic black phosphorus. Physical Review B, 2010. 82(13).

71. Bridgman, P.W., Effects of High Shearing Stress Combined with High Hydrostatic Pressure. Physical Review, 1935. 48(10): p. 825-847.

72. Warschauer, D., Electrical and Optical Properties of Crystalline Black Phosphorus. Journal of Applied Physics, 1963. 34(7): p. 1853-1860.

73. Brown, A. and S. Rundqvist, Refinement of the crystal structure of black phosphorus. Acta Crystallographica, 1965. 19(4): p. 684-685.

74. Shoichi, E., et al., Growth of Large Single Crystals of Black Phosphorus under High Pressure. Japanese Journal of Applied Physics, 1982. 21(8A): p. L482.

75. Liu, H., et al., Semiconducting black phosphorus: synthesis, transport properties and electronic applications. Chemical Society Reviews, 2015. 44(9): p. 2732-2743.

76. Sansone, G., et al., Towards an Accurate Estimate of the Exfoliation Energy of Black Phosphorus: a Periodic Quantum Chemical Approach. The journal of physical chemistry letters, 2015.

77. Liu, H., et al., Semiconducting black phosphorus: synthesis, transport properties and electronic applications. Chemical Society Reviews, 2015. 44(9): p. 2732-2743.

78. Park, C.M. and H.J. Sohn, Black Phosphorus and its Composite for Lithium Rechargeable Batteries. Advanced Materials, 2007. 19(18): p. 2465-2468.

79. Nilges, T., M. Kersting, and T. Pfeifer, A fast low-pressure transport route to large black phosphorus single crystals. Journal of Solid State Chemistry, 2008. 181(8): p. 1707-1711.

80. Köpf, M., et al., Access and in situ growth of phosphorene-precursor black phosphorus. Journal of Crystal Growth, 2014. 405: p. 6-10.

81. Samuel Thomas Moran, A.N., Mark Barron, Umair Khakoo, Deji Akinwande, Investigating Ultrasonicated Allotropes of Phosphorus. 2015.

82. Novoselov, K.S., et al., Two-dimensional gas of massless Dirac fermions in graphene. Nature, 2005. 438(7065): p. 197-200.

83. Liu, H., et al., Phosphorene: An Unexplored 2D Semiconductor with a High Hole Mobility. Acs Nano, 2014. 8(4): p. 4033-4041.

84. Li, L., et al., Black phosphorus field-effect transistors. Nature Nanotechnology, 2014. 9(5): p. 372-377.

85. Kuanchen, X., L. Xi, and J.C.M. Hwang. Phosphorene FETs - Promising transistors based on a few layers of phosphorus atoms. in Advanced Materials and Processes for RF and THz Applications (IMWS-AMP), 2015 IEEE MTT-S International Microwave Workshop Series on. 2015.

86. Das, S., et al., Tunable Transport Gap in Phosphorene. Nano Letters, 2014. 14(10): p. 5733-5739.

87. Das, S., M. Demarteau, and A. Roelofs, Ambipolar Phosphorene Field Effect Transistor. Acs Nano, 2014. 8(11): p. 11730-11738.

88. Buscema, M., et al., Fast and Broadband Photoresponse of Few-Layer Black Phosphorus Field-Effect Transistors. Nano Letters, 2014. 14(6): p. 3347-3352. 
89. Castellanos-Gomez, A., et al., Isolation and characterization of few-layer black phosphorus. 2D Materials, 2014. 1(2).

90. Xia, F., H. Wang, and Y. Jia, Rediscovering black phosphorus as an anisotropic layered material for optoelectronics and electronics. Nature communications, 2014. 5.

91. Yang, Z., et al., Field-Effect Transistors Based on Amorphous Black Phosphorus Ultrathin Films by Pulsed Laser Deposition. Advanced Materials, 2015. 27(25): p. 37483754.

92. Castellanos-Gomez, A., Black Phosphorus: Narrow Gap, Wide Applications. Journal of Physical Chemistry Letters, 2015. 6(21): p. 4280-4291.

93. Cain, D.F. and R.E. Davies, Breakdown of adenosine triphosphate during a single contraction of working muscle. Biochemical and Biophysical Research Communications, 1962. 8(5): p. 361-366.

94. Srivastava, A., et al., Unique electron transport in ultrathin black phosphorene: Ab-initio study. Applied Surface Science, 2015. 356: p. 881-887.

95. Fei, R. and L. Yang, Strain-Engineering the Anisotropic Electrical Conductance of FewLayer Black Phosphorus. Nano Letters, 2014. 14(5): p. 2884-2889.

96. Ong, Z.-Y., et al., Strong Thermal Transport Anisotropy and Strain Modulation in SingleLayer Phosphorene. Journal of Physical Chemistry C, 2014. 118(43): p. 25272-25277.

97. Qiao, J., et al., High-mobility transport anisotropy and linear dichroism in few-layer black phosphorus. Nature Communications, 2014. 5.

98. Vy, T., et al., Layer-controlled band gap and anisotropic excitons in few-layer black phosphorus. Physical Review B, 2014. 89(23).

99. Cai, Y., et al., Giant Phononic Anisotropy and Unusual Anharmonicity of Phosphorene: Interlayer Coupling and Strain Engineering. Advanced Functional Materials, 2015.

25(15): p. 2230-2236.

100. Chaves, A., et al., Anisotropic exciton Stark shift in black phosphorus. Physical Review B, 2015. 91(15).

101. Ge, S., et al., Dynamical Evolution of Anisotropic Response in Black Phosphorus under Ultrafast Photoexcitation. Nano Letters, 2015. 15(7): p. 4650-4656.

102. Ghazaryan, A. and T. Chakraborty, Aspects of anisotropic fractional quantum Hall effect in phosphorene. Physical Review B, 2015.92(16).

103. He, J., et al., Exceptional and Anisotropic Transport Properties of Photocarriers in Black Phosphorus. Acs Nano, 2015. 9(6): p. 6436-6442.

104. Jain, A. and A.J.H. McGaughey, Strongly anisotropic in-plane thermal transport in single-layer black phosphorene. Scientific Reports, 2015. 5.

105. Kou, L., et al., Anisotropic Ripple Deformation in Phosphorene. Journal of Physical Chemistry Letters, 2015. 6(9): p. 1509-1513.

106. Luo, Z., et al., Anisotropic in-plane thermal conductivity observed in few-layer black phosphorus. Nature Communications, 2015. 6.

107. Popovic, Z.S., J.M. Kurdestany, and S. Satpathy, Electronic structure and anisotropic Rashba spin-orbit coupling in monolayer black phosphorus. Physical Review B, 2015. 92(3).

108. Qin, G., et al., Anisotropic intrinsic lattice thermal conductivity of phosphorene from first principles. Physical Chemistry Chemical Physics, 2015. 17(7): p. 4854-4858.

109. Schuster, R., et al., Anisotropic Particle-Hole Excitations in Black Phosphorus. Physical Review Letters, 2015. 115(2).

110. Tao, J., et al., Mechanical and Electrical Anisotropy of Few-Layer Black Phosphorus. Acs Nano, 2015. 9(11): p. 11362-11370.

111. Wang, L., et al., Electro-mechanical anisotropy of phosphorene. Nanoscale, 2015. 7(21): p. 9746-9751. 
112. Wang, X., et al., Highly anisotropic and robust excitons in monolayer black phosphorus. Nature Nanotechnology, 2015. 10(6): p. 517-521.

113. Wang, Z. and P.X.L. Feng, Design of black phosphorus $2 D$ nanomechanical resonators by exploiting the intrinsic mechanical anisotropy. 2d Materials, 2015. 2(2).

114. Island, J.O., et al., Environmental instability of few-layer black phosphorus. 2d Materials, 2015. 2(1).

115. Wood, J.D., et al., Effective Passivation of Exfoliated Black Phosphorus Transistors against Ambient Degradation. Nano Letters, 2014. 14(12): p. 6964-6970.

116. Favron, A., et al., Photooxidation and quantum confinement effects in exfoliated black phosphorus. Nature Materials, 2015. 14(8): p. 826-+.

117. Guo, Z.N., et al., From Black Phosphorus to Phosphorene: Basic Solvent Exfoliation, Evolution of Raman Scattering, and Applications to Ultrafast Photonics. Advanced Functional Materials, 2015. 25(45): p. 6996-7002.

118. Liu, H., et al., The Effect of Dielectric Capping on Few-Layer Phosphorene Transistors: Tuning the Schottky Barrier Heights. Ieee Electron Device Letters, 2014. 35(7): p. 795797.

119. Kresse, G. and J. Furthmüller, Efficient iterative schemes for ab initio total-energy calculations using a plane-wave basis set. Physical Review B, 1996. 54(16): p. 1116911186.

120. Kohn, W., A.D. Becke, and R.G. Parr, Density Functional Theory of Electronic Structure. The Journal of Physical Chemistry, 1996. 100(31): p. 12974-12980.

121. Kohn, W. and L.J. Sham, Self-Consistent Equations Including Exchange and Correlation Effects. Physical Review, 1965. 140(4A): p. A1133-A1138.

122. Perdew, J.P., K. Burke, and M. Ernzerhof, Generalized Gradient Approximation Made Simple. Physical Review Letters, 1996. 77(18): p. 3865-3868.

123. Jones, R.O. and O. Gunnarsson, The density functional formalism, its applications and prospects. Reviews of Modern Physics, 1989. 61(3): p. 689-746.

124. Kim, K. and K.D. Jordan, Comparison of Density Functional and MP2 Calculations on the Water Monomer and Dimer. The Journal of Physical Chemistry, 1994. 98(40): p. 10089-10094.

125. Balendhran, S., et al., Elemental Analogues of Graphene: Silicene, Germanene, Stanene, and Phosphorene. Small, 2015. 11(6): p. 640-652.

126. Heyd, J., G.E. Scuseria, and M. Ernzerhof, Hybrid functionals based on a screened Coulomb potential. Journal of Chemical Physics, 2003. 118(18): p. 8207-8215.

127. Li, P. and I. Appelbaum, Electrons and holes in phosphorene. Physical Review B, 2014. 90(11): p. 115439.

128. Hu, W., et al., Edge-Modified Phosphorene Nanoflake Heterojunctions as Highly Efficient Solar Cells. Nano Letters, 2016.

129. Gillespie, R.J., The VSEPR model revisited. Chemical Society Reviews, 1992. 21(1): p. 59-69.

130. Atapattu Mudiyaneselage, R.J., In-situ monitoring of transport properties of graphene during plasma functionalization and it's applications in energy storage. 2013, University of Louisville: Louisville, Kentucky :.

131. Ziletti, A., et al., Phosphorene oxides: Bandgap engineering of phosphorene by oxidation. Physical Review B, 2015. 91(8): p. 085407.

132. Cai, Y., G. Zhang, and Y.-W. Zhang, Layer-dependent Band Alignment and Work Function of Few-Layer Phosphorene. Scientific Reports, 2014. 4.

133. Lee, Y.H., et al., Synthesis of Large-Area MoS2 Atomic Layers with Chemical Vapor Deposition. Advanced Materials, 2012. 24(17): p. 2320-2325.

134. Elias, A.L., et al., Controlled Synthesis and Transfer of Large-Area WS2 Sheets: From Single Layer to Few Layers. Acs Nano, 2013. 7(6): p. 5235-5242. 
135. Xia, J., et al., CVD synthesis of large-area, highly crystalline MoSe2 atomic layers on diverse substrates and application to photodetectors. Nanoscale, 2014. 6(15): p. 89498955.

136. Lv, R., et al., Transition Metal Dichalcogenides and Beyond: Synthesis, Properties, and Applications of Single- and Few-Layer Nanosheets. Accounts of Chemical Research, 2015. 48(1): p. 56-64.

137. Geim, A.K. and K.S. Novoselov, The rise of graphene. Nature Materials, 2007. 6(3): p. 183-191.

138. Bao, W., et al., High mobility ambipolar MoS2 field-effect transistors: Substrate and dielectric effects. Applied Physics Letters, 2013. 102(4).

139. Li, X., et al., Large-Area Synthesis of High-Quality and Uniform Graphene Films on Copper Foils. Science, 2009. 324(5932): p. 1312-1314.

140. Zhan, Y., et al., Large-Area Vapor-Phase Growth and Characterization of MoS2 Atomic Layers on a SiO2 Substrate. Small, 2012. 8(7): p. 966-971.

141. van der Zande, A.M., et al., Grains and grain boundaries in highly crystalline monolayer molybdenum disulphide. Nature Materials, 2013. 12(6): p. 554-561.

142. Ramakrishna Matte, H.S.S., et al., MoS2 and WS2 Analogues of Graphene. Angewandte Chemie International Edition, 2010. 49(24): p. 4059-4062.

143. Zeng, Z., et al., Single-Layer Semiconducting Nanosheets: High-Yield Preparation and Device Fabrication. Angewandte Chemie-International Edition, 2011. 50(47): p. 1109311097.

144. Coleman, J.N., et al., Two-Dimensional Nanosheets Produced by Liquid Exfoliation of Layered Materials. Science, 2011. 331(6017): p. 568-571.

145. Cunningham, G., et al., Solvent Exfoliation of Transition Metal Dichalcogenides: Dispersibility of Exfoliated Nanosheets Varies Only Weakly between Compounds. Acs Nano, 2012. 6(4): p. 3468-3480.

146. Brent, J.R., et al., Production of few-layer phosphorene by liquid exfoliation of black phosphorus. Chemical Communications, 2014. 50(87): p. 13338-13341.

147. Yasaei, P., et al., High-Quality Black Phosphorus Atomic Layers by Liquid-Phase Exfoliation. Advanced Materials, 2015. 27(11): p. 1887-+.

148. Jayasingha, R., et al., In Situ Study of Hydrogenation of Graphene and New Phases of Localization between Metal-Insulator Transitions. Nano Letters, 2013. 13(11): p. 50985105.

149. Hashmi, A., U. Farooq, and J. Hong, Graphene/phosphorene bilayer: High electron speed, optical property and semiconductor-metal transition with electric field. Current Applied Physics, 2016. 16(3): p. 318-323.

150. Chen, X., et al., High-quality sandwiched black phosphorus heterostructure and its quantum oscillations. Nature Communications, 2015. 6.

151. Elías, A.L., et al., Controlled Synthesis and Transfer of Large-Area WS $W_{2}$ Sheets: From Single Layer to Few Layers. Acs Nano, 2013. 7(6): p. 5235-5242.

152. Laursen, A.B., et al., Molybdenum sulfides-efficient and viable materials for electro - and photoelectrocatalytic hydrogen evolution. Energy \& Environmental Science, 2012. 5(2): p. 5577-5591.

153. Fang, H., et al., Degenerate n-Doping of Few-Layer Transition Metal Dichalcogenides by Potassium. Nano Letters, 2013. 13(5): p. 1991-1995.

154. Lv, R., et al., Nitrogen-doped graphene: beyond single substitution and enhanced molecular sensing. Scientific Reports, 2012. 2.

155. Fang, H., et al., High-Performance Single Layered WSe2 p-FETs with Chemically Doped Contacts. Nano Letters, 2012. 12(7): p. 3788-3792. 
156. Wang, H.B., et al., Structure and magnetism of Mn, Fe, or Co adatoms on monolayer and bilayer black phosphorus. Journal of Magnetism and Magnetic Materials, 2016. 401: p. 706-710.

157. Yang, L., W. Mi, and X. Wang, Tailoring magnetism of black phosphorene doped with B, $C, N, O, F, S$ and Se atom: A DFT calculation. Journal of Alloys and Compounds, 2016. 662: p. 528-533.

158. Edmonds, M.T., et al., Creating a Stable Oxide at the Surface of Black Phosphorus. Acs Applied Materials \& Interfaces, 2015. 7(27): p. 14557-14562.

159. Lu, J.P., et al., Bandgap Engineering of Phosphorene by Laser Oxidation toward Functional 2D Materials. Acs Nano, 2015. 9(10): p. 10411-10421.

160. Huo, N., et al., Photoresponsive and Gas Sensing Field-Effect Transistors based on Multilayer WS2 Nanoflakes. Scientific Reports, 2014. 4.

161. Ding, B., et al., Tuning Phosphorene Nanoribbon Electronic Structure through Edge Oxidization. The Journal of Physical Chemistry C, 2016.

162. Mishchenko, A., et al., Nonlocal Response and Anamorphosis: The Case of Few-Layer Black Phosphorus. Nano Letters, 2015. 15(10): p. 6991-6995.

163. Ribeiro, H.B., et al., Unusual Angular Dependence of the Raman Response in Black Phosphorus. Acs Nano, 2015. 9(4): p. 4270-4276.

164. Sun, J., et al., A phosphorene-graphene hybrid material as a high-capacity anode for sodium-ion batteries. Nat Nano, 2015. 10(11): p. 980-985.

165. Xie, Y.Q., et al., Photogalvanic effect in monolayer black phosphorus. Nanotechnology, 2015. 26(45).

166. Yuan, H., et al., Polarization-sensitive broadband photodetector using a black phosphorus vertical p-n junction. Nature Nanotechnology, 2015. 10(8): p. 707-713.

167. Zhou, X.Y., et al., Anomalous magneto-optical response of black phosphorus thin films.

Physical Review B, 2015. 92(16).

168. Cao, T.F., et al., Electric field and strain tunable electronic structures in monolayer Black Phosphorus. Computational Materials Science, 2016. 112: p. 297-303.

169. Gaoxue, W., et al., Out-of-plane structural flexibility of phosphorene. Nanotechnology, 2016. 27(5): p. 055701.

170. Sun, J., et al., Formation of Stable Phosphorus-Carbon Bond for Enhanced Performance in Black Phosphorus Nanoparticle-Graphite Composite Battery Anodes. Nano Letters, 2014. 14(8): p. 4573-4580.

171. Li, W.F., et al., Ultrafast and Directional Diffusion of Lithium in Phosphorene for HighPerformance Lithium-Ion Battery. Nano Letters, 2015. 15(3): p. 1691-1697.

172. Yao, Q.S., et al., Theoretical Prediction of Phosphorene and Nanoribbons As FastCharging Li Ion Battery Anode Materials. Journal of Physical Chemistry C, 2015. 119(12): p. 6923-6928.

173. Stan, M.C., et al., Puzzling out the origin of the electrochemical activity of black $P$ as a negative electrode material for lithium-ion batteries. Journal of Materials Chemistry A, 2013. 1(17): p. 5293-5300.

174. F Xu, B.G., J Chen, L Huo, H Ma, C Zhu, W Xia, H Min, Z Li, S Li, K Yu, F Wang, Y Zhu, L Wu, Y Cui, L Sun, , Shear-Exfoliated Phosphorene for Rechargeable Nanoscale Battery. arXiv preprint arXiv:1411.6124, 2015.

175. Yu, Z., et al., Phosphorus-Graphene Nanosheet Hybrids as Lithium-Ion Anode with Exceptional High-Temperature Cycling Stability. Advanced Science, 2015. 2(1-2): p. n/an/a.

176. Zhang, R., X. Wu, and J. Yang, Blockage of ultrafast and directional diffusion of Li atoms on phosphorene with intrinsic defects. Nanoscale, 2016. 8(7): p. 4001-4006.

177. Radisavljevic, B., et al., Single-layer MoS2 transistors. Nature Nanotechnology, 2011. 6(3): p. 147-150. 
178. Alam, K. and R.K. Lake, Monolayer MoS2 Transistors Beyond the Technology Road Map. Ieee Transactions on Electron Devices, 2012. 59(12): p. 3250-3254.

179. Kim, S., et al., High-mobility and low-power thin-film transistors based on multilayer MoS2 crystals. Nature Communications, 2012. 3.

180. Li, T., Ideal strength and phonon instability in single-layer MoS2. Physical Review B, 2012. 85(23).

181. Hanlon, D., et al., Liquid exfoliation of solvent-stabilized few-layer black phosphorus for applications beyond electronics. Nature Communications, 2015. 6.

182. Sreshtt, V., A.A.H. Padua, and D. Blankschtein, Liquid-Phase Exfoliation of Phosphorene: Design Rules from Molecular Dynamics Simulations. Acs Nano, 2015. 9(8): p. 8255-8268.

183. Knudsen, M., Die maximale verdampfungsgeschwindigkeit des quecksilbers. Annalen der Physik, 1915. 352(13): p. 697-708.

184. Tonndorf, P., et al., Photoluminescence emission and Raman response of monolayer MoS2, MoSe2, and WSe2. Optics Express, 2013. 21(4): p. 4908-4916.

185. Zhang, S., et al., Extraordinary Photoluminescence and Strong Temperature/AngleDependent Raman Responses in Few-Layer Phosphorene. Acs Nano, 2014. 8(9): p. 9590-9596.

186. Kopf, M., et al., Access and in situ growth of phosphorene-precursor black phosphorus. Journal of Crystal Growth, 2014. 405: p. 6-10.

187. Raman, C.V., Krishnan, K.S., A new type of secondary radiation. Nature, 1928. 121: p. 501-502.

188. Lord Rayleigh, J.W.S., On the light from the sky, its polarization and color. Philosophical Magazine, 1871. 41: p. 447-54.

189. Dumcenco, D.O., et al., Raman study of 2H-Mol-xWxS2 layered mixed crystals. Journal of Alloys and Compounds, 2010. 506(2): p. 940-943.

190. Castellanos-Gomez, A., et al., Isolation and characterization of few-layer black phosphorus. 2D Materials, 2014. 1(2): p. 19.

191. Zhang, S., et al., Extraordinary Photoluminescence and Strong Temperature/AngleDependent Raman Responses in Few-Layer Phosphorene. Acs Nano, 2014. 8(9): p. 9590-9596.

192. Endo, S., et al., GROWTH OF LARGE SINGLE-CRYSTALS OF BLACK PHOSPHORUS UNDER HIGH-PRESSURE. Japanese Journal of Applied Physics Part 2-Letters, 1982. 21(8): p. L482-L484.

193. Takao, Y., H. Asahina, and A. Morita, ELECTRONIC-STRUCTURE OF BLACK PHOSPHORUS IN TIGHT-BINDING APPROACH. Journal of the Physical Society of Japan, 1981. 50(10): p. 3362-3369.

194. Crichton, W.A., et al., Phosphorus: New in situ powder data from large-volume apparatus. Powder Diffraction, 2003. 18(2): p. 155-158.

195. Liu, H., et al., Phosphorene: An Unexplored 2D Semiconductor with a High Hole Mobility. Acs Nano, 2014. 8(4): p. 4033-4041.

196. Keyes, R.W., Fundamental limits of silicon technology. Proceedings of the Ieee, 2001. 89(3): p. 227-239.

197. Wang, X., et al., High-responsivity graphene/silicon-heterostructure waveguide photodetectors. Nature Photonics, 2013. 7(11): p. 888-891.

198. Du, H.W., et al., Recent developments in black phosphorus transistors. Journal of Materials Chemistry C, 2015. 3(34): p. 8760-8775.

199. Zhang, S., et al., Extraordinarily bound quasi-one-dimensional trions in two-dimensional phosphorene atomic semiconductors. arXiv preprint arXiv:1411.6124, 2014.

200. Ameen, T.A., et al., Few-layer Phosphorene: An Ideal 2D Material For Tunnel Transistors. arXiv preprint arXiv:1512.05021, 2015. 
201. Mukhopadhyay, A., et al., Effect of stacking order on device performance of bilayer black phosphorene-field-effect transistor. Journal of Applied Physics, 2015. 118(22): p. 224501.

202. Yang, Z.B., et al., Field-Effect Transistors Based on Amorphous Black Phosphorus Ultrathin Films by Pulsed Laser Deposition. Advanced Materials, 2015. 27(25): p. 37483754.

203. Cai, Y.Q., et al., Energetics, Charge Transfer, and Magnetism of Small Molecules Physisorbed on Phosphorene. Journal of Physical Chemistry C, 2015. 119(6): p. 31023110. 


\section{CURRICULUM VITAE}

Name:

Address:

Telephone:

Email:

Date of Birth:

Place of Birth:

Citizenship:

Nationality:

Gender:

$8 / 2008-5 / 2010$
George Anderson

3501 Dorset Rd.

Louisville, KY 40214

614-946-1008

gmande05@yahoo.com

9/10/1984

Westerville, Ohio, U.S.A.

U.S.A.

African American

Male

\section{Employment History}

Physics Tutor, Eastern Kentucky University Physics Department.

Tutor college and high school students in Physics. 
8/2009 - 5/2010 Assistant Instructor, Eastern Kentucky University Physics

Department; Assist main instructors with teaching/laboratory duties and responsible for teaching class if main professor absent.

8/2010 - 5/2012 Graduate Teaching Assistant, University of Louisville Physics Department.

6/2012 - 8/2012 Theoretical Researcher, Shanghai University; Shanghai, China; Conducted theoretical research on carbon fullerene nanostructures to determine electronic properties and structural stability. Research funded by National Science Foundation through East Asia Pacific Summer Institute in collaboration with China

8/2012 - Present Graduate Research Assistant, University of Louisville; Louisville, KY; Developed laboratory for thin film/semiconductor/nano-scale material fabrication and analysis. Assembling and testing laboratory equipment, including multi-zone quartz tube furnaces and thermal evaporators. Designed, built, and maintain vacuum systems for deposition processes. Materials development and characterization. Etch processes using Potassium Hydroxide and cleaning processes using Piranha solution. Developing Metal Oxide Semiconductor Field Effect Transistors (MOSFET) based on single atom monolayer channel. Fabrication and analysis of monolayer based heterostructures grown by thermal evaporation, chemical vapor deposition, and laser/plasma assisted deposition processes.

Education

8/1999 - 5/2003 Mifflin High school, Columbus, Ohio 
8/2003 - 5/2004 Ohio State University, Columbus, Ohio; Major-Mechanical

Engineering

8/2004 - 12/2007 Eastern Kentucky University, Richmond, Kentucky; Major-

Physics Engineering

8/2008 - 5/2010 Eastern Kentucky University, Richmond, Kentucky; Major-

Physics Engineering; Degree Earned-Bachelors of Science, Physics Engineering

8/2010 - 12/2012 University of Louisville, Louisville, Kentucky; Graduate School; Major-Physics; Degree Earned-Masters of Science, Physics

12/2012 - 12/2015 University of Louisville, Louisville, Kentucky; Graduate School; Major- Physics, PhD. Expected Graduation 12/2015

\section{Professional Qualifications}

Equipment/Training: Chemical Vapor Deposition, Thermal Evaporation, High Temperature Torch Glass Sealing (Blowing), Thin Film Growth (Semiconductor, Insulator, Metal), Scanning Electron Microscopy (SEM), Energy-Dispersive X-Ray Spectroscopy, X-Ray Diffraction (XRD), Raman Spectroscopy, Photoluminescence, Atomic Force Microscopy, X-Ray Photoelectron Spectroscopy, Ultra-Violet Photoelectron Spectroscopy, UV-Visible Spectroscopy (Absorption, Transmission), Electrical Circuit Design and Implementation, Two Probe Electrical Measurement (I-V, Gate Dependence), Hydraulic Lifts, Impact Tools, Soldering, Vacuum Pump (Mechanical, Diffusion, Turbo) Installation and Maintenance, Vacuum System Design, Multi and Signal Zone Quartz Tube Furnace, Ultrasonic Baths, Optical Microscopes 
Publications and Presentations

Patterned 2D Heterostructures Based on Transition Metal Dichalcogenides. George Anderson $^{1}$, Sreyansh Agarwal ${ }^{1}$, Jacek B. Jasinski ${ }^{3}$ and Humberto R. Gutiérrez ${ }^{1,2}$, ${ }^{1}$ Department of Physics and Astronomy; University of Louisville, Louisville, KY 40292; ${ }^{2}$ Department of Physics, University of South Florida, Tampa, FL 33620; ${ }^{3}$ Conn Center for Renewable Energy Research, University of Louisville. To be submitted

Recent advances in Synthesis, Characterization, and Applications of Phosphorene. George Anderson ${ }^{1}$, Meysam Akhtar ${ }^{1}$, Rong Zhao $^{1}$, Adel Alruqi ${ }^{1}$, Gamini Sumanasekera ${ }^{1,2}$, Jacek B. Jasinski ${ }^{1,2}$, Joanna E. Mroczkowska, ${ }^{1}$ Department of Physics and Astronomy; University of Louisville, Louisville, KY 40292; ${ }^{2}$ Conn Center for Renewable Energy Research, University of Louisville. To be submitted

In-situ Environmental Dependent Transport Properties in Phosphorene .George Anderson ${ }^{1}$, Rong Zhao ${ }^{1}$, Adel Alruqi ${ }^{1}$, Jacek B. Jasinski ${ }^{2}$ and Gamini Sumanesekera ${ }^{1,2}$, ${ }^{1}$ Department of Physics and Astronomy; University of Louisville, Louisville, KY 40292; ${ }^{2}$ Conn Center for Renewable Energy Research, University of Louisville. To be submitted Light Induced Variable Transport in Phosphorene. George Anderson ${ }^{1}$, *, Rong Zhao ${ }^{1}$, Adel Alruqi ${ }^{1}$, Jacek B. Jasinski ${ }^{2}$ and Gamini Sumanesekera ${ }^{1,2},{ }^{1}$ Department of Physics and Astronomy; University of Louisville, Louisville, KY 40292; ${ }^{2}$ Conn Center for Renewable Energy Research, University of Louisville. To be submitted

Pressure dependent Raman and Photoluminescence in Phosphorene. Meysam Akhtar ${ }^{1}$, George Anderson ${ }^{1}$, Rong Zhao ${ }^{1}$, Adel Alruqi ${ }^{1}$, Jacek B. Jasinski ${ }^{2}$ and Gamini 
Sumanesekera ${ }^{1,2},{ }^{1}$ Department of Physics and Astronomy; University of Louisville, Louisville, KY 40292; ${ }^{2}$ Conn Center for Renewable Energy Research, University of Louisville. In process

Phosphorene based anode for Li-Ion battery applications. Ruchira Dharmasena ${ }^{1}$, George Anderson ${ }^{1}$, Meysam Akhtar ${ }^{1}$, Rong Zhao ${ }^{1}$, Adel Alruqi ${ }^{1}$, Jacek B. Jasinski ${ }^{2}$ and Gamini Sumanesekera ${ }^{1,2},{ }^{1}$ Department of Physics and Astronomy; University of Louisville, Louisville, KY 40292; ${ }^{2}$ Conn Center for Renewable Energy Research, University of Louisville. In process

University of Louisville Alumni Fellow Dinner Student Speaker. 10-22-15

GEM Grad Lab Poster Presentation. Presented poster of research and talked with minority undergraduate students to encourage them to go to graduate school. 9-19-14. 